\title{
Substituted Benzothietes: Synthesis and a Quantum Chemical Investigation of their Cycloreversion Properties
}

\section{Supporting Information}

Nils L. Ahlburg, ${ }^{\dagger}$ Andres Raul Velarde, ${ }^{\ddagger}$ Matthew Kieber-Emmons, ${ }^{\ddagger}$ Peter G. Jones, ${ }^{\S}$ and Daniel B. Werz ${ }^{\dagger *}$ ${ }^{\dagger}$ Technische Universität Braunschweig, Institute of Organic Chemistry and ${ }^{\S}$ Technische Universität Braunschweig, Institute of Inorganic and Analytical Chemistry, Hagenring 30, 38106 Braunschweig, Germany

${ }^{\ddagger}$ University of Utah, Department of Chemistry, Salt Lake City, Utah 84112-0850, United States of America

\section{Contents}

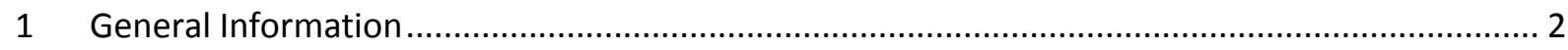

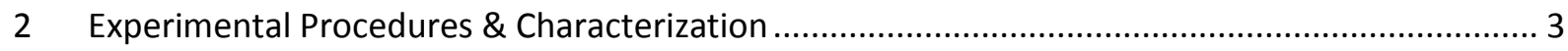

2.1 General Procedure for Synthesis of 2-Mercapto Pivalophenones from

2-Mercaptobenzonitriles

2.2 General Procedure for the Protection of 2-Mercapto Aldehydes and Ketones................... 4

2.3 General Procedure for the Synthesis of Benzothietes ........................................... 5

$2.4 \quad$ Large Scale Synthesis of Benzothiete 9 a ................................................................ 5

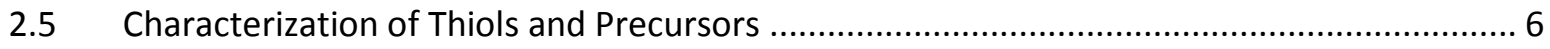

2.6 Characterization of Carbamothioates .............................................................. 14

2.7 Characterization of Benzothietes ................................................................... 19

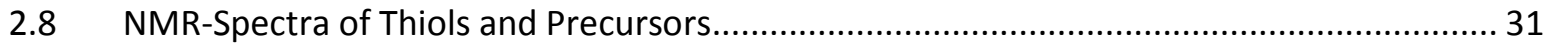

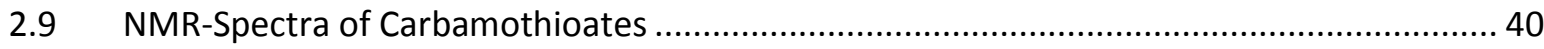

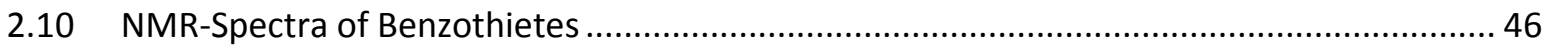

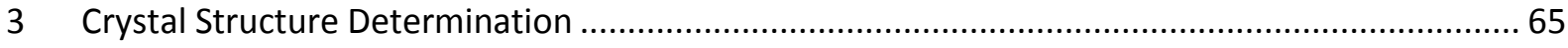

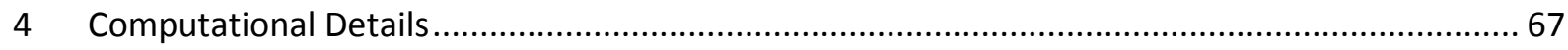

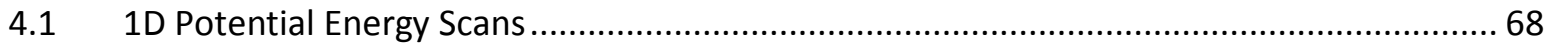

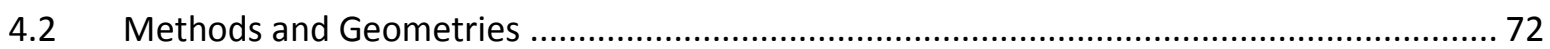

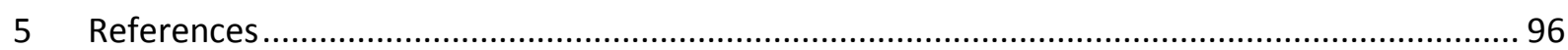




\section{General Information}

All solvents were purchased as synthetic grade solvents and stored over molecular sieves. All reactions were carried out in oven-dried glassware, septum-capped and under atmospheric pressure of argon unless otherwise stated. Commercially available compounds were used without further purification unless otherwise stated. Flash column chromatography was conducted on Merck Geduran ${ }^{\circledR} \mathrm{Si} 60$ silicagel $(43-60 \mu \mathrm{m})$, for thin layer chromatography Merck TLC silica gel $60 \mathrm{~F}_{254}$ aluminum sheets were used exclusively. Cooling to $0{ }^{\circ} \mathrm{C}$ was achieved with regular water/ice/ $\mathrm{NaCl}$ baths. For lower temperatures, a Julabo FT902 mobile cryostat was used to cool an acetone-filled Dewar bowl into which the reaction vessel was immersed. ESI-HRMS mass spectrometry was carried out on a FTICR instrument, El-HRMS mass spectrometry was carried out on JOEL AccuTOF GC JMS-T100GC instrument. For reaction control and yield determination, a GC-MS (7820A GC System+5977E MSD) by Agilent Technologies was employed. IR spectra were recorded on a Bruker Tensor 27 ATR spectrometer. Proton $\left({ }^{1} \mathrm{H}\right)$ and carbon $\left({ }^{13} \mathrm{C}\right)$ NMR spectra were recorded on a Bruker Avance II 300, a Bruker DRX 400, a Bruker Avance II 400 and a Bruker Avance II 600 spectrometer using the signals from tetramethylsilane as internal references for ${ }^{1} \mathrm{H}$ and ${ }^{13} \mathrm{C}$ chemical shifts, respectively. Abbreviations for multiplicities were used as following $s=$ singlet, $d=$ doublet, $t=$ triplet, $q=$ quartet, $p=$ quintet, hept $=$ septet, $\mathrm{m}=$ multiplet. 


\section{Experimental Procedures \& Characterization}

The 2-mercapto aldehydes and ketones were prepared according to known procedures $\left(\mathbf{a}^{1}, \mathbf{b}^{2}, \mathbf{c}^{3}, \mathbf{d}^{4}\right.$, $\mathbf{e}^{5}$ ). General procedures are given for critical steps of the precursor synthesis, such as the synthesis of 2-mercapto pivalophenones from nitriles (2.1) and protection of free thiols (2.2). The analytical data of known products was consistent with the corresponding references $\left(1 \mathbf{a}^{6}, \mathbf{3}^{7}, \mathbf{1 3}^{1}, \mathbf{1 4}^{1}, \mathbf{1 6}^{2}, \mathbf{1 7}^{2}, \mathbf{1 9}^{3}\right.$, $\left.\mathbf{2 1} \mathbf{1}^{4}, \mathbf{2 4 ^ { 5 }}, \mathbf{2 5 ^ { 8 }}, \mathbf{2 6 ^ { 5 }}, \mathbf{2} \mathbf{7}^{9}\right)$. Previously unreported compounds were fully characterized and the data is given in the respective section. Scheme S1 provides an overview of the used procedures

Scheme S1. Synthetic Overview.

(a)

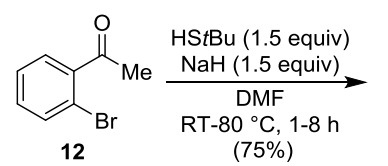

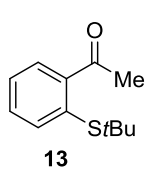

13

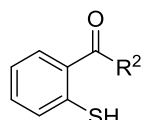

$\mathrm{R}^{2}=\mathrm{Ph} 16(64 \%)$

$\mathrm{R}^{2}=t \mathrm{Bu} 17(42 \%)$

$n$ BuLi (2 equiv)

(c)

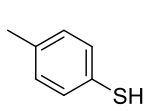

18

$$
\begin{aligned}
& \text { TMEDA (2 equiv) } \\
& \stackrel{\text { PhCN (1 equiv) }}{\mathrm{CyH}}, \\
& 0^{\circ} \mathrm{C}-\mathrm{RT}, 24 \mathrm{~h}
\end{aligned}
$$
aq. workup $(47 \%)$

$n$ BuLi (2 equiv) TMEDA ( 2 equiv)

(d)

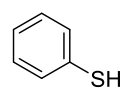
\begin{tabular}{l} 
DMF ( 1 equiv) \\
\hline
\end{tabular}

20

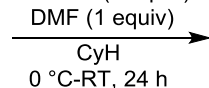

aq. workup aq. workup<smiles>Cc1cccc(S)c1C(=O)c1ccccc1</smiles>

19

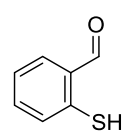

21 (e)<smiles>[R][X]c1ccc(C#N)c([N+](=O)[O-])c1</smiles>
$\mathrm{R}^{1}=\mathrm{CF}_{3} 23$

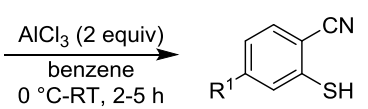

(see 2.1)
$\mathrm{R}^{1}=\mathrm{Me} 26(67 \%)$ $\mathrm{R}^{1}=\mathrm{CF}_{3} 27(86 \%)$

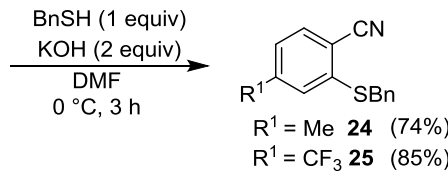

$\mathrm{R}^{1}=\mathrm{CF}_{3} 25(85 \%)$

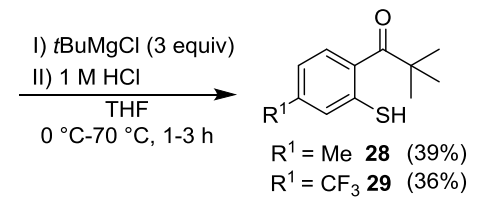

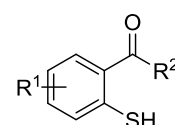
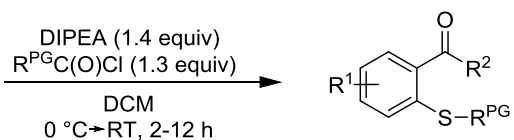

$\mathbf{R}^{1}=\mathbf{H} ; \mathbf{R}^{2}=\mathbf{H}$

\begin{tabular}{|llll|}
$\mathrm{R}^{\mathrm{PG}}=\mathrm{C}(\mathrm{O}) \mathrm{NMe} \mathrm{e}_{2}$ & $\mathbf{2 1 \rightarrow 1 a}(65 \%)$ & $\mathrm{R}^{\mathrm{PG}}=\mathrm{C}(\mathrm{O}) \mathrm{OMe}$ & $\mathbf{2 1 \rightarrow 1 e}(70 \%)$ \\
$\mathrm{R}^{\mathrm{PG}}=\mathrm{C}(\mathrm{O}) \mathrm{O}(\mathrm{PMP})$ & $\mathbf{2 1 \rightarrow 1 b}(73 \%)$ & $\mathrm{R}^{\mathrm{PG}}=\mathrm{Troc}$ & $\mathbf{2 1 \rightarrow 1 f}(70 \%)$ \\
$\mathrm{R}^{\mathrm{PG}}=\mathrm{Cbz}$ & $\mathbf{2 1 \rightarrow 1 c}(70 \%)$ & $\mathrm{R}^{\mathrm{PG}}=\mathrm{C}(\mathrm{O}) \mathrm{OPh}$ & $\mathbf{2 1 \rightarrow 1 g}(30 \%)$ \\
$\mathrm{R}^{P G}=$ Alloc & $\mathbf{2 1 \rightarrow 1 d}(33 \%)$ & & \\
\hline
\end{tabular}

$\mathbf{R}^{\mathrm{PG}}=\mathrm{C}(\mathrm{O}) \mathrm{NMe}_{2}$

$\mathrm{R}^{1}=\mathrm{H} ; \quad \mathrm{R}^{2}=\mathrm{Me} 14 \rightarrow 3 \quad(65 \%) \quad \mathrm{R}^{1}=4-\mathrm{Me} ; \mathrm{R}^{2}=t \mathrm{Bu} \quad \mathbf{2 8} \rightarrow \mathbf{7} \quad(68 \%)$

$\mathrm{R}^{1}=\mathrm{H} ; \quad \mathrm{R}^{2}=\mathrm{Ph} \quad \mathbf{1 6 \rightarrow 4} \quad(70 \%) \quad \mathrm{R}^{1}=4-\mathrm{CF}_{3} ; \mathrm{R}^{2}=t \mathrm{Bu} 29 \rightarrow \mathbf{8} \quad(55 \%)$

$R^{1}=\mathrm{H} ; \quad \mathrm{R}^{2}=t \mathrm{Bu} 17 \rightarrow 5 \quad(72 \%)$

$R^{1}=5-M e ; R^{2}=P h \quad 19 \rightarrow 6 \quad(48 \%)$ 


\subsection{General Procedure for Synthesis of 2-Mercapto Pivalophenones from 2-Mercaptobenzonitriles}<smiles>[R]c1ccc(C#N)c(S)c1</smiles>

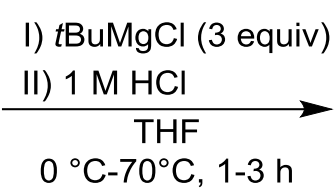<smiles>[R]c1ccc(C(=O)C(C)(C)C)c(S)c1</smiles>

To a solution of the thiol (10.0 mmol, 1.00 equiv) in THF $(100 \mathrm{~mL})$ was added $t \mathrm{BuMgCl}(1.7 \mathrm{M}$ in THF, $17.6 \mathrm{~mL}, 30.0 \mathrm{mmol}, 3.0$ equiv) at $0{ }^{\circ} \mathrm{C}$ (water/ice/ $\mathrm{NaCl}$ mixture). After $30 \mathrm{~min}$ the cooling bath was removed and the solution was allowed to warm to room temperature. The solution was heated to $70^{\circ} \mathrm{C}$ (oil bath) until full conversion of the thiol was observed (GC-MS control). The reaction was cooled to $0{ }^{\circ} \mathrm{C}$ again (water/ice/ $\mathrm{NaCl}$ mixture) and was quenched with $1 \mathrm{M} \mathrm{HCl}(30 \mathrm{~mL})$. The ice bath was removed again and stirring was continued until the imine was fully hydrolyzed. The mixture was diluted with $\mathrm{Et}_{2} \mathrm{O}$ and then washed three times with $0.5 \mathrm{M} \mathrm{HCl}$. The organic layer was dried over $\mathrm{MgSO}_{4}$ and filtered. The solvent was removed and the residue was purified by flash column chromatography (100/1/1=pentane/Et ${ }_{2} \mathrm{O} /$ formic acid; silicagel $43-60 \mu \mathrm{m}$ ).

Note: Varying amounts of thiol were used.

\subsection{General Procedure for the Protection of 2-Mercapto Aldehydes and Ketones}<smiles>[R]C(=O)c1c[R1]ccc1S</smiles>
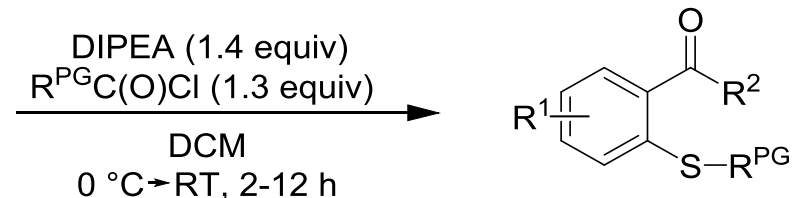

To a solution of the thiol $(2.00 \mathrm{mmol}, 1.00$ equiv) in DCM $(50 \mathrm{~mL})$ was added Hünig base $(0.488 \mathrm{~mL}$, $2.80 \mathrm{mmol}, 1.4$ equiv) at $0{ }^{\circ} \mathrm{C}$ (water/ice/ $\mathrm{NaCl}$ mixture). The respective carbonyl chloride $(2.60 \mathrm{mmol}$, 1.3 equiv) was added portionwise over $15 \mathrm{~min}$. After $30 \mathrm{~min}$ the cooling bath was removed and the solution was allowed to warm to room temperature. The stirring was continued until full conversion of the thiol was achieved (GC-MS control). The reaction was quenched with $1 \mathrm{M} \mathrm{HCl}(50 \mathrm{~mL})$. The aqueous phase was extracted three times with DCM $(25 \mathrm{~mL})$. The combined organic layers were dried over $\mathrm{MgSO}_{4}$ and filtered. The solvent was removed, the residue was purified by flash column chromatography (100/1 to 50/1=DCM/MeOH; silicagel 43 - $60 \mu \mathrm{m})$.

Note: Varying amounts of thiol were used. 


\subsection{General Procedure for the Synthesis of Benzothietes}<smiles>[R][R]1ccc(S(=O)(=O)OC)c(C([R])=O)c1</smiles>

To a solution of dimethylcarbamothioate $\left(0.20 \mathrm{mmol}, 1.0\right.$ equiv) in THF $(5 \mathrm{~mL})$ at $-20{ }^{\circ} \mathrm{C}$ (mobile cryostat, acetone bath) was added Grignard reagent $(0.60 \mathrm{mmol}, 3.0$ equiv) at a rate of $0.40 \mathrm{mmol} / \mathrm{min}$. The reaction was stirred for $20 \mathrm{~min}$ and then warmed to the indicated temperature (oil bath, s. main text). After full conversion had been achieved (GC-MS control), excess Grignard reagent was quenched with a drop of $i \mathrm{PrOH}$, the solvent was removed and the residue was purified by flash column chromatography (pentane/diethyl ether=100/1; silicagel $43-60 \mu \mathrm{m}$ ).

\subsection{Large Scale Synthesis of Benzothiete 9a}

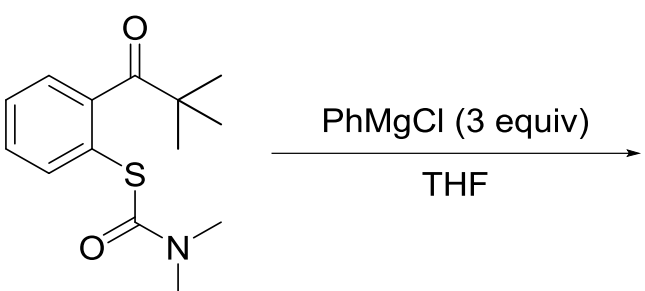

5

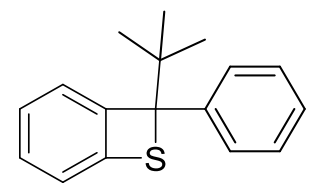

$9 a$

To a solution of dimethylcarbamothioate $5(0.27 \mathrm{~g}, 1.00 \mathrm{mmol}, 1.0$ equiv) in THF ( $25 \mathrm{~mL})$ at $-20^{\circ} \mathrm{C}$ (mobile cryostat, acetone bath) was added $\mathrm{PhMgCl}(2 \mathrm{M}, 1.5 \mathrm{~mL}, 3.00 \mathrm{mmol}, 3.0$ equiv) at a rate of $0.40 \mathrm{mmol} / \mathrm{min}$. The reaction was stirred for $20 \mathrm{~min}$ and then warmed to room temperature for $1.5 \mathrm{~h}$. Excess Grignard reagent was quenched with a drop of $\mathrm{PrOH}$, the solvent was removed and the residue was purified by flash column chromatography (pentane/ethyl acetate $=100 / 1, \mathrm{R}_{\mathrm{f}}=0.60$; silicagel 43 $60 \mu \mathrm{m})$. The product $9 \mathrm{a}$ was obtained as colorless crystals $(0.16 \mathrm{~g}, 0.61 \mathrm{mmol}, 61 \%)$. 


\subsection{Characterization of Thiols and Precursors}

Note: The quaternary aromatic carbon attached to the formyl group was not visible in the ${ }^{13} \mathrm{C}-\mathrm{NMR}$ spectra of compounds $\mathbf{1 b}$ to $\mathbf{1 g}$.

\subsubsection{S-(2-Formylphenyl) O-(4-Methoxyphenyl) Carbonothioate (1b)}<smiles>COc1ccc(OC(=O)Sc2ccccc2C=O)cc1</smiles>

Yield: $420 \mathrm{mg}, 73 \%$.

Appearance: Pale brown solid.

$\mathrm{R}_{\mathrm{f}}=0.20$ (25/1=pentane/ethyl acetate)

${ }^{1} \mathrm{H}-\mathrm{NMR}\left(500 \mathrm{MHz}, \mathrm{CDCl}_{3}\right): \delta=10.49(\mathrm{~d}, J=0.6 \mathrm{~Hz}, 1 \mathrm{H}), 8.05-8.03(\mathrm{~m}, 1 \mathrm{H}), 7.73-7.70(\mathrm{~m}, 1 \mathrm{H}), 7.66-$ $7.62(m, 1 \mathrm{H}), 7.62-7.59(\mathrm{~m}, 1 \mathrm{H}), 7.11-7.08(\mathrm{~m}, 2 \mathrm{H}), 6.88-6.86(\mathrm{~m}, 2 \mathrm{H}), 3.78(\mathrm{~s}, 3 \mathrm{H})$.

${ }^{13} \mathrm{C}-\mathrm{NMR}\left(125 \mathrm{MHz}, \mathrm{CDCl}_{3}\right): \delta=190.5,167.8,157.7,156.7,144.7,137.0,134.2,130.7,130.4,129.8$, $121.9,114.5,55.6$.

IR (ATR): $\tilde{v}\left[\mathrm{~cm}^{-1}\right]=2839,1716,1690,1592,1502,1187,1113,1110,1026$.

$m p=56-57^{\circ} \mathrm{C}$

HRMS (ESI, pos.) m/z: 


\subsubsection{O-Benzyl S-(2-Formylphenyl) Carbonothioate (1c)}<smiles>O=Cc1ccccc1SC(=O)OCc1ccccc1</smiles>

Yield: $379 \mathrm{mg}, \mathbf{7 0 \%}$.

Appearance: Colorless liquid.

$\mathrm{R}_{\mathrm{f}}=0.16(50 / 1=$ pentane/ethyl acetate)

${ }^{1} \mathrm{H}-\mathrm{NMR}\left(500 \mathrm{MHz}, \mathrm{CDCl}_{3}\right): \delta=10.41(\mathrm{~d}, J=0.7 \mathrm{~Hz}, 1 \mathrm{H}), 8.02-8.00(\mathrm{~m}, 1 \mathrm{H})$, 7.64-7.54 $(\mathrm{m}, 3 \mathrm{H}), 7.38-$ $7.33(\mathrm{~m}, 5 \mathrm{H}), 5.25(\mathrm{~s}, 2 \mathrm{H})$.

${ }^{13} \mathrm{C}-\mathrm{NMR}\left(125 \mathrm{MHz}, \mathrm{CDCl}_{3}\right): \delta=190.6,168.3,136.9,134.5,134.1,130.8,130.5,129.3,128.8,128.6$, 128.5, 70.1 .

IR (ATR): $\tilde{v}\left[\mathrm{~cm}^{-1}\right]=1717,1689,1584,1133,1112$.

$m p=55-57^{\circ} \mathrm{C}$

HRMS (ESI, pos.) m/z:

$[\mathrm{M}+\mathrm{Na}]^{+}$Calcd. for $\mathrm{C}_{15} \mathrm{H}_{12} \mathrm{O}_{3} \mathrm{SNa}$

Found
295.0405;

295.0402 . 


\subsubsection{O-Allyl S-(2-Formylphenyl) Carbonothioate (1d)}<smiles>C=CCOC(=O)Sc1ccccc1C=O</smiles>

Yield: $146 \mathrm{mg}, 33 \%$.

Appearance: Colorless liquid.

$R_{f}=0.20$ (50/1=pentane/ethyl acetate)

${ }^{1} \mathrm{H}-\mathrm{NMR}\left(500 \mathrm{MHz}, \mathrm{CDCl}_{3}\right): \delta=10.48(\mathrm{~d}, J=0.9 \mathrm{~Hz}, 1 \mathrm{H}), 8.05-8.02(\mathrm{~m}, 1 \mathrm{H}), 7.67-7.57(\mathrm{~m}, 3 \mathrm{H})$, 5.97-5.87 (m, $1 \mathrm{H}), 5.38-5.28(\mathrm{~m}, 2 \mathrm{H}), 4.73(\mathrm{dt}, J=7.4,1.7 \mathrm{~Hz}, 2 \mathrm{H})$.

${ }^{13} \mathrm{C}-\mathrm{NMR}\left(125 \mathrm{MHz}, \mathrm{CDCl}_{3}\right): \delta=190.7,168.1,137.0,134.1,130.9,130.8,130.6,129.4,119.9,69.0$. IR (ATR): $\tilde{v}\left[\mathrm{~cm}^{-1}\right]=1721,1695,1132,1111$.

HRMS (ESI, pos.) m/z: 


\subsubsection{S-(2-Formylphenyl) O-Methyl Carbonothioate (1e)}<smiles>COC(=O)Sc1ccccc1C=O</smiles>

Appearance: Pale yellow liquid.

Yield: $276 \mathrm{mg}, 70 \%$.

$R_{f}=0.16(50 / 1=$ pentane/ethyl acetate)

${ }^{1} \mathrm{H}-\mathrm{NMR}\left(500 \mathrm{MHz}, \mathrm{CDCl}_{3}\right): \delta=10.44(\mathrm{~d}, J=0.9 \mathrm{~Hz}, 1 \mathrm{H}), 9.05-8.02(\mathrm{~m}, 2 \mathrm{H}), 7.66-7.58(\mathrm{~m}, 3 \mathrm{H})$, $3.87(\mathrm{~s}, 3 \mathrm{H})$.

${ }^{13} \mathrm{C}-\mathrm{NMR}\left(125 \mathrm{MHz}, \mathrm{CDCl}_{3}\right): \delta=190.7,168.9,137.0,134.1,130.8,130.6,129.4,55.1$.

IR (ATR): $\tilde{v}\left[\mathrm{~cm}^{-1}\right]=1715,1690,1129$.

HRMS (ESI, pos.) $\mathrm{m} / \mathrm{z}$ :

$[\mathrm{M}+\mathrm{Na}]^{+} \mathrm{Calcd}$. for $\mathrm{C}_{9} \mathrm{H}_{8} \mathrm{O}_{3} \mathrm{SNa}$

219.0092;

Found 219.0090 


\subsubsection{S-(2-Formylphenyl) O-(2,2,2-Trichloroethyl) Carbonothioate (1f)}<smiles>O=Cc1ccccc1SC(=O)OCC(Cl)(Cl)Cl</smiles>

Yield: $442 \mathrm{mg}, 70 \%$.

Appearance: Colorless liquid.

$\mathrm{R}_{\mathrm{f}}=0.20$ (50/1=pentane/ethyl acetate)

${ }^{1} \mathrm{H}-\mathrm{NMR}\left(400 \mathrm{MHz}, \mathrm{CDCl}_{3}\right): \delta=10.46(\mathrm{~d}, J=0.6 \mathrm{~Hz}, 1 \mathrm{H}), 8.07-8.04(\mathrm{~m}, 1 \mathrm{H}), 7.71-7.63(\mathrm{~m}, 3 \mathrm{H}), 4.85(\mathrm{~s}$, $2 \mathrm{H})$.

${ }^{13} \mathrm{C}-\mathrm{NMR}\left(100 \mathrm{MHz}, \mathrm{CDCl}_{3}\right): \delta=190.4,167.9,137.1,137.0,134.3,131.0,129.9,129.6,94.0,76.1$.

IR (ATR): $\tilde{v}\left[\mathrm{~cm}^{-1}\right]=1729,1696,1586,1266,1198,1105,1052$.

HRMS (ESI, pos.) m/z:

$[\mathrm{M}+\mathrm{Na}]^{+}$Calcd. for $\mathrm{C}_{10} \mathrm{H}_{7} \mathrm{Cl}_{3} \mathrm{O}_{3} \mathrm{SNa}$

334.9079;

Found

334.9077. 


\subsubsection{S-(2-Formylphenyl) O-Phenyl Carbonothioate (1g)}<smiles>O=Cc1ccccc1SC(=O)Oc1ccccc1</smiles>

Appearance: Pale brown solid.156 mg, 30\%.

$\mathrm{R}_{\mathrm{f}}=0.18$ (50/1=pentane/ethyl acetate)

${ }^{1} \mathrm{H}-\mathrm{NMR}\left(400 \mathrm{MHz}, \mathrm{CDCl}_{3}\right): \delta=10.50(\mathrm{~d}, J=0.6 \mathrm{~Hz}, 1 \mathrm{H}), 8.05-8.04(\mathrm{~m}, 1 \mathrm{H}), 7.74-7.72(\mathrm{~m}, 1 \mathrm{H}), 7.68-$ 7.60 (m, $2 \mathrm{H}), 7.40-7.35$ (m, $2 \mathrm{H}), 7.27-7.22(\mathrm{~m}, 1 \mathrm{H}), 7.20-7.17$ (m, $2 \mathrm{H})$.

${ }^{13} \mathrm{C}-\mathrm{NMR}\left(100 \mathrm{MHz}, \mathrm{CDCl}_{3}\right): \delta=190.5,167.5,151.2,137.0,136.9,134.2,130.8,130.2,129.9,126.5$, 121.0.

IR (ATR): $\tilde{v}\left[\mathrm{~cm}^{-1}\right]=1690,1651,1585,1472,1183,1157,1099$.

$m p=57-59^{\circ} \mathrm{C}$

HRMS (ESI, pos.) m/z:

$[\mathrm{M}+\mathrm{Na}]^{+}$Calcd. for $\mathrm{C}_{14} \mathrm{H}_{10} \mathrm{O}_{3} \mathrm{SNa}$

Found
281.0248;

281.0245 . 
2.5.1.7 1-(2-Mercapto-4-methylphenyl)-2,2-dimethylpropan-1-one (28)<smiles>Cc1ccc(C(=O)C(C)(C)C)c(S)c1</smiles>

Yield: $812 \mathrm{mg}, 39 \%$.

Appearance: Pale yellow liquid.

$\mathrm{R}_{\mathrm{f}}=0.30\left(100 / 1 / 1=\right.$ pentane/Et ${ }_{2} \mathrm{O} /$ formic acid $)$

${ }^{1} \mathrm{H}-\mathrm{NMR}\left(500 \mathrm{MHz}, \mathrm{CDCl}_{3}\right): \delta=7.23(\mathrm{~d}, J=7.9 \mathrm{~Hz}, 1 \mathrm{H}), 7.19(\mathrm{~s}, 1 \mathrm{H}), 6.98-6.96(\mathrm{~m}, 1 \mathrm{H}), 3.57(\mathrm{~s}, 1 \mathrm{H})$, $2.31(\mathrm{~s}, 3 \mathrm{H}), 1.30(\mathrm{~s}, 9 \mathrm{H})$.

${ }^{13} \mathrm{C}-\mathrm{NMR}\left(125 \mathrm{MHz}, \mathrm{CDCl}_{3}\right): \delta=212.2,139.7,137.2,132.6,129.0,126.3,125.9,45.0,27.5,21.1$.

IR (ATR): $\tilde{v}\left[\mathrm{~cm}^{-1}\right]=2966,2927,2870,1683,1597,1472,1390,1365,1280,1190,1148,1074$.

HRMS (ESI, pos.) m/z:

$[\mathrm{M}+\mathrm{Na}]^{+}$Calcd. for $\mathrm{C}_{12} \mathrm{H}_{16} \mathrm{OSNa}$

231.0820;

Found

231.0815 . 
<smiles>CC(C)(C)C(=O)c1ccc(C(F)(F)F)cc1S</smiles>

Yield: $656 \mathrm{mg}, 25 \%$.

Appearance: Pale yellow liquid.

$\mathrm{R}_{\mathrm{f}}=0.20$ (100/1/1=pentane/Et ${ }_{2} \mathrm{O} /$ formic acid $)$

${ }^{1} \mathrm{H}-\mathrm{NMR}\left(500 \mathrm{MHz}, \mathrm{CDCl}_{3}\right): \delta=7.64-7.63(\mathrm{~m}, 1 \mathrm{H}), 7.45-7.43(\mathrm{~m}, 1 \mathrm{H}), 7.34(\mathrm{~d}, J=8.00 \mathrm{~Hz}, 1 \mathrm{H}), 3.68(\mathrm{~s}$, $1 \mathrm{H}), 1.30(\mathrm{~s}, 9 \mathrm{H})$.

${ }^{13} \mathrm{C}-\mathrm{NMR}\left(125 \mathrm{MHz}, \mathrm{CDCl}_{3}\right): \delta=212.0,144.0$ (d, $J=1 \mathrm{~Hz}, 1 \mathrm{C}$ ), 131.4 (q, $J=33 \mathrm{~Hz}, 1 \mathrm{C}$ ), 129.6, 128.7 (d, $J=4 \mathrm{~Hz}, 1 \mathrm{C}$ ), 126.1, 123.3 (q, J = 273 Hz, 1 C), 122.2 (q, J = $4 \mathrm{~Hz}, 1 \mathrm{C}$, ), 45.2, 27.1.

${ }^{19} \mathrm{~F}-\mathrm{NMR}\left(376 \mathrm{MHz}, \mathrm{CDCl}_{3}\right): \delta=-63.5$.

IR (ATR): $\tilde{v}\left[\mathrm{~cm}^{-1}\right]=2972,1689,1480,1390,1261,1171,1127,1077$.

HRMS (ESI, pos.) $\mathrm{m} / \mathrm{z}: \quad[\mathrm{M}+\mathrm{Na}]^{+}$Calcd. for $\mathrm{C}_{12} \mathrm{H}_{13} \mathrm{~F}_{3} \mathrm{OSNa}$

285.0537;

Found

285. 0531. 


\subsection{Characterization of Carbamothioates}

\subsubsection{S-(2-Benzoylphenyl) Dimethylcarbamothioate (4)}<smiles>CN(C)C(=O)Sc1ccccc1C(=O)c1ccccc1</smiles>

Yield: $850 \mathrm{mg}, 70 \%$.

Appearance: Colorless solid.

$\mathrm{R}_{\mathrm{f}}=0.15(200 / 1=\mathrm{DCM} / \mathrm{MeOH})$

${ }^{1} \mathrm{H}-\mathrm{NMR}\left(400 \mathrm{MHz}, \mathrm{CDCl}_{3}\right): \delta=7.81-7.79(\mathrm{~m}, 2 \mathrm{H}), 7.66-7.64(\mathrm{~m}, 1 \mathrm{H}), 7.58-7.40(\mathrm{~m}, 6 \mathrm{H}), 2.86(\mathrm{~s}, 6 \mathrm{H})$.

${ }^{13} \mathrm{C}-\mathrm{NMR}\left(100 \mathrm{MHz}, \mathrm{CDCl}_{3}\right): \delta=196.7,165.7,144.2,138.2,137.3,133.1,130.3,130.2,128.9,128.4$, $128.2,127.2,36.9$.

IR (ATR): $\tilde{v}\left[\mathrm{~cm}^{-1}\right]=1663,1363,1287,1252,1091,1057$.

$\mathrm{mp}=83-85^{\circ} \mathrm{C}$

HRMS (ESI, pos.) m/z: 


\subsubsection{S-(2-Pivaloylphenyl) Dimethylcarbamothioate (5)}<smiles>CN(C)C(=O)Sc1ccccc1C(=O)C(C)(C)C</smiles>

Yield: $700 \mathrm{mg}, \mathbf{7 2} \%$.

Appearance: Colorless solid.

$R_{f}=0.10(200 / 1=D C M / M e O H)$

${ }^{1} \mathrm{H}-\mathrm{NMR}\left(500 \mathrm{MHz}, \mathrm{CDCl}_{3}\right): \delta=7.58-7.56(\mathrm{~m}, 1 \mathrm{H})$, 7.43-7.37 (m, $\left.2 \mathrm{H}\right), 7.21-7.19(\mathrm{~m}, 1 \mathrm{H}), 3.05(\mathrm{~s}, 3 \mathrm{H})$, $3.01(\mathrm{~s}, 3 \mathrm{H}), 1.24(\mathrm{~s}, 9 \mathrm{H})$.

${ }^{13} \mathrm{C}-\mathrm{NMR}\left(125 \mathrm{MHz}, \mathrm{CDCl}_{3}\right): \delta=212.9,165.6,146.1,138.3,128.9,128.5,125.0,124.9,45.0,37.0,27.3$. IR (ATR): $\tilde{v}\left[\mathrm{~cm}^{-1}\right]=2971,2933,2871,166,1581,1477,1462,1365,1259,1193$.

$\mathrm{mp}=78-80^{\circ} \mathrm{C}$

$\operatorname{HRMS}(E S I$, pos.) $\mathrm{m} / \mathrm{z}$ :

$[\mathrm{M}+\mathrm{Na}]^{+}$Calcd. for $\mathrm{C}_{14} \mathrm{H}_{19} \mathrm{NO}_{2} \mathrm{SNa}$

288.1034;

Found

288.1030. 


\subsubsection{S-(2-Benzoyl-4-methylphenyl) Dimethylcarbamothioate (6)}<smiles>Cc1ccc(SC(=O)N(C)C)c(C(=O)c2ccccc2)c1</smiles>

Yield: $950 \mathrm{mg}, 48 \%$.

Appearance: Colorless solid.

$R_{f}=0.10(200 / 1=D C M / M e O H)$

${ }^{1} \mathrm{H}-\mathrm{NMR}\left(400 \mathrm{MHz}, \mathrm{CDCl}_{3}\right): \delta=7.81-7.79(\mathrm{~m}, 2 \mathrm{H})$, 7.57-7.50 (m, $\left.2 \mathrm{H}\right)$, 7.44-7.40 (m, $\left.2 \mathrm{H}\right)$, 7.35-7.32 (m, $1 \mathrm{H}), 7.22-7.21(\mathrm{~m}, 1 \mathrm{H}), 2.84(\mathrm{~s}, 6 \mathrm{H}), 2.40(\mathrm{~s}, 3 \mathrm{H})$.

${ }^{13} \mathrm{C}-\mathrm{NMR}\left(100 \mathrm{MHz}, \mathrm{CDCl}_{3}\right): \delta=197.0,166.0,144.3,139.5,138.2,137.4,133.0,131.2,130.2,128.9$, $128.2,123.6,36.8,21.2$.

IR (ATR): $\tilde{v}\left[\mathrm{~cm}^{-1}\right]=1659,1592,1448,1359,1289,1250,1090,1055$.

$m p=93-95^{\circ} \mathrm{C}$

HRMS (ESI, pos.) m/z:

$[\mathrm{M}+\mathrm{Na}]^{+}$Calcd. for $\mathrm{C}_{17} \mathrm{H}_{17} \mathrm{NO}_{2} \mathrm{SNa}$

322.0878;

Found

322.0874 


\subsubsection{S-(5-Methyl-2-pivaloylphenyl) Dimethylcarbamothioate (7)}<smiles>Cc1ccc(C(=O)C(C)(C)C)c(SC(=O)N(C)C)c1</smiles>

Yield: $380 \mathrm{mg}, 68 \%$.

Appearance: Pale yellow liquid.

$R_{f}=0.35(100 / 1=D C M / M e O H)$

${ }^{1} \mathrm{H}-\mathrm{NMR}\left(500 \mathrm{MHz}, \mathrm{CDCl}_{3}\right): \delta=7.39(\mathrm{~s}, 1 \mathrm{H}), 7.20-7.17(\mathrm{~m}, 1 \mathrm{H}), 7.10(\mathrm{~d}, J=7.8 \mathrm{~Hz}, 1 \mathrm{C}), 3.03(\mathrm{~s}, 3 \mathrm{H})$, $2.37(\mathrm{~s}, 3 \mathrm{H}), 1.23(\mathrm{~s}, 9 \mathrm{H})$.

${ }^{13} \mathrm{C}-\mathrm{NMR}\left(125 \mathrm{MHz}, \mathrm{CDCl}_{3}\right): \delta=212.9,165.6,143.3,138.9,138.6,129.3,124.9,124.6,44.9,36.9,27.3$, 21.1.

IR (ATR): $\tilde{v}\left[\mathrm{~cm}^{-1}\right]=2966,2929,1669,1600,1474,1360,1258,1094,1064$.

HRMS (ESI, pos.) m/z: $\quad[\mathrm{M}+\mathrm{Na}]^{+}$Calcd. for $\mathrm{C}_{15} \mathrm{H}_{21} \mathrm{NO}_{2} \mathrm{SNa}$

302.1191;

Found

302.1187. 
<smiles>CN(C)C(=O)Sc1cc(C(F)(F)F)ccc1C(=O)C(C)(C)C</smiles>

Yield: $367 \mathrm{mg}, 55 \%$.

Appearance: Colorless solid.

$R_{f}=0.40(100 / 1=D C M / M e O H)$

${ }^{1} \mathrm{H}-\mathrm{NMR}\left(500 \mathrm{MHz}, \mathrm{CDCl}_{3}\right): \delta=7.89-7.86(\mathrm{~m}, 1 \mathrm{H}), 7.64-7.62(\mathrm{~m}, 1 \mathrm{H}), 7.31(\mathrm{~d}, J=7.97 \mathrm{~Hz}, 1 \mathrm{H}), 3.06(\mathrm{~s}$, $3 \mathrm{H}), 3.03(\mathrm{~s}, 3 \mathrm{H}), 1.25(\mathrm{~s}, 9 \mathrm{H})$.

${ }^{13} \mathrm{C}-\mathrm{NMR}\left(125 \mathrm{MHz}, \mathrm{CDCl}_{3}\right): \delta=211.8,164.4,149.2,135.1$ (q, $\left.J=4 \mathrm{~Hz}, 1 \mathrm{C}\right), 131.2$ (q, J= $4 \mathrm{~Hz}, 1 \mathrm{C}$ ) 1 (q, $J=33 \mathrm{~Hz}, 1 \mathrm{C}), 126.6,125.5,125.4(q, J=4 \mathrm{~Hz}, 1 \mathrm{C}), 123.4$ (q, $J=273 \mathrm{~Hz}, 1 \mathrm{C}), 45.0,37.2,37.0,27.2$.

IR (ATR): $\tilde{v}\left[\mathrm{~cm}^{-1}\right]=2974,1683,1663,1363,1322,1258,1197,1121,1074$.

${ }^{19} \mathrm{~F}-\mathrm{NMR}\left(376 \mathrm{MHz}, \mathrm{CDCl}_{3}\right): \delta=-63.1$.

$\mathrm{mp}=76-78^{\circ} \mathrm{C}$

HRMS (ESI, pos.) $\mathrm{m} / \mathrm{z}$ : 


\subsection{Characterization of Benzothietes}

\subsubsection{8-Phenyl-7-thiabicyclo[4.2.0]octa-1,3,5-triene (2)}<smiles>c1ccc(C2Sc3ccccc32)cc1</smiles>

Yield: see below.

Appearance: Colorless liquid.

Compound 2 was isolated from a large scale reaction $(2 \mathrm{mmol})$, it was purified by column chromatography on a short silica plug $(10 \mathrm{~cm}$; silicagel $43-60 \mu \mathrm{m})$. It quickly decomposes upon exposure to the acidic material. The solvent was removed and the sample immediately transferred to a freezer $\left(-30^{\circ} \mathrm{C}\right)$, analyzed and used for GC-MS yield calibration. Yield: $55.5 \mathrm{mg}, 14 \%$ (isolated), $75 \%$ (GC-MS).

$\mathrm{R}_{\mathrm{f}}=0.50\left(100 / 1=\right.$ pentane $\left./ \mathrm{Et}_{2} \mathrm{O}\right)$

${ }^{1} \mathrm{H}-\mathrm{NMR}\left(500 \mathrm{MHz}, \mathrm{CDCl}_{3}\right): \delta=7.72-7.71(\mathrm{~m}, 2 \mathrm{H}), 7.44-7.41(\mathrm{~m}, 2 \mathrm{H}), 7.34(\mathrm{tt}, J=7.4,1.9 \mathrm{~Hz}, 1 \mathrm{H}), 7.23$ (dd, $J=7.7,1.4 \mathrm{~Hz}, 1 \mathrm{H}$ ), 7.09 (s, $1 \mathrm{H}$ ), 7.03 (td, $J=7.5,1.4 \mathrm{~Hz}, 1 \mathrm{H}), 6.95$ (dd, $J=7.9,1.5 \mathrm{~Hz}, 1 \mathrm{H}), 6.83$ (td, $J=7.5,1.5 \mathrm{~Hz}, 1 \mathrm{H})$.

${ }^{13} \mathrm{C}-\mathrm{NMR}\left(125 \mathrm{MHz}, \mathrm{CDCl}_{3}\right): \delta=144.5,139.5,135.0,133.2,129.4,129.0,128.5,128.4,128.2,127.7$, 126.7, 53.4.

IR (ATR): $\tilde{v}\left[\mathrm{~cm}^{-1}\right]=2970,2901,1516,1280,1162$.

HRMS (El, pos., 70eV) m/z:

$\mathrm{M}^{+\cdot}$ Calcd. for $\mathrm{C}_{13} \mathrm{H}_{10} \mathrm{~S}$

198.0503;

Found 198.0508 . 


\subsubsection{8-(tert-Butyl)-8-phenyl-7-thiabicyclo[4.2.0]octa-1,3,5-triene (9a)}

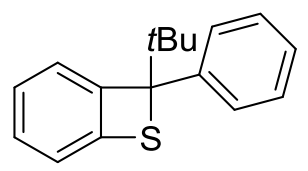

Yield: $33.1 \mathrm{mg}, 65 \%$.

Appearance: Colorless solid.

$R_{f}=0.40\left(100 / 1=\right.$ pentane $\left./ E_{2} O\right)$

Recorded NMR spectra are in accordance with existing literature. ${ }^{10}$

${ }^{1} \mathrm{H}-\mathrm{NMR}\left(500 \mathrm{MHz}, \mathrm{CDCl}_{3}\right): \delta=7.53-7.51(\mathrm{~m}, 2 \mathrm{H}), 7.30-7.20(\mathrm{~m}, 5 \mathrm{H}), 7.13(\mathrm{td}, J=7.6,1.0 \mathrm{~Hz}, 1 \mathrm{H}), 7.01$ $(\mathrm{dt}, J=7.6,0.9 \mathrm{~Hz}, 1 \mathrm{H}), 1.10(\mathrm{~s}, 9 \mathrm{H})$.

${ }^{13} \mathrm{C}-\mathrm{NMR}\left(125 \mathrm{MHz}, \mathrm{CDCl}_{3}\right): \delta=145.1,140.1,140.1,129.5,128.8,126.6,126.6,123.7,123.2,121.6$, $81.1,38.1,26.8$.

Note: The $\mathrm{CDCl}_{3}$-peak is overlaying a multiplet of aromatic protons, thus the depicted integral is too large. 


\subsubsection{8-(tert-Butyl)-8-(4-methoxyphenyl)-7-thiabicyclo[4.2.0]octa-1,3,5-triene (9b)}

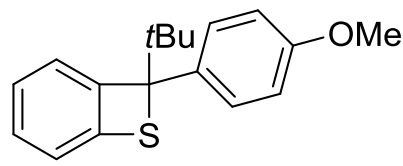

Yield: $35.3 \mathrm{mg}, 62 \%$.

Appearance: Colorless liquid.

$R_{f}=0.25\left(100 / 1=\right.$ pentane $\left./ E_{2} \mathrm{O}\right)$

Recorded NMR spectra are in accordance with existing literature. ${ }^{10}$

${ }^{1} \mathrm{H}-\mathrm{NMR}\left(400 \mathrm{MHz}, \mathrm{CDCl}_{3}\right): \delta=7.45-7.42(\mathrm{~m}, 2 \mathrm{H}), 7.23-7.19(\mathrm{~m}, 2 \mathrm{H}), 7.12(\mathrm{td}, J=7.6,1.0 \mathrm{~Hz}, 1 \mathrm{H})$, $7.01(\mathrm{dt}, J=7.5,1.0 \mathrm{~Hz}, 1 \mathrm{H}), 6.82-6.80(\mathrm{~m}, 2 \mathrm{H}), 3.79(\mathrm{~s}, 3 \mathrm{H}), 1.09(\mathrm{~s}, 9 \mathrm{H})$.

${ }^{13} \mathrm{C}-\mathrm{NMR}\left(100 \mathrm{MHz}, \mathrm{CDCl}_{3}\right): \delta=158.2,145.2,140.0,132.3,130.6,128.7,123.6,123.0,121.7,112.0$, $80.9,55.3,38.3,26.7$. 


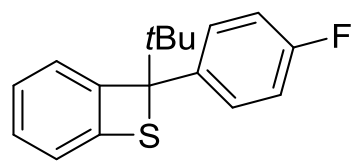

Yield: $35.4 \mathrm{mg}, 65 \%$.

Appearance: Colorless liquid.

$R_{f}=0.35\left(100 / 1=\right.$ pentane/Et $\left.{ }_{2} \mathrm{O}\right)$

${ }^{1} \mathrm{H}-\mathrm{NMR}\left(400 \mathrm{MHz}, \mathrm{CDCl}_{3}\right): \delta=7.50-7.47(\mathrm{~m}, 2 \mathrm{H}), 7.25-7.21(\mathrm{~m}, 2 \mathrm{H}), 7.13(\mathrm{td}, J=8.1,1.8 \mathrm{~Hz}, 1 \mathrm{H}), 7.04-$ $7.01(\mathrm{~m}, 1 \mathrm{H}), 6.99-6.93(\mathrm{~m}, 2 \mathrm{H}), 1.08(\mathrm{~s}, 9 \mathrm{H})$.

${ }^{13} \mathrm{C}-\mathrm{NMR}\left(100 \mathrm{MHz}, \mathrm{CDCl}_{3}\right): \delta=161.6$ (d, $\left.J=246 \mathrm{~Hz}, 1 \mathrm{C}\right), 144.9,139.9,136.0$ (d, $J=3 \mathrm{~Hz}, 1 \mathrm{C}$ ), 131.0 (d, $J=8 \mathrm{~Hz}, 1 \mathrm{C}), 129.0,123.8,123.0,121.7,113.4(\mathrm{~d}, J=21 \mathrm{~Hz}, 1 \mathrm{C}), 80.3,38.1,26.7$.

${ }^{19} \mathrm{~F}-\mathrm{NMR}\left(376 \mathrm{MHz}, \mathrm{CDCl}_{3}\right): \delta=-116.6$.

IR (ATR): $\tilde{v}\left[\mathrm{~cm}^{-1}\right]=2958,2927,2867,1506,1443,1229,1162$.

HRMS (El, pos., 70eV) m/z: $\quad \mathrm{M}^{+\bullet}$ Calcd. for $\mathrm{C}_{17} \mathrm{H}_{17} \mathrm{FS}$ 272.1035;

Found 272.1042 . 


\subsubsection{8-(tert-Butyl)-8-(p-toluyl)-7-thiabicyclo[4.2.0]octa-1,3,5-triene (9d)}

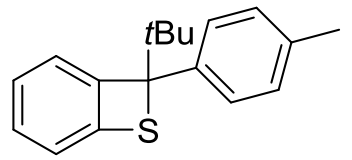

Yield: $37.6 \mathrm{mg}, 70 \%$.

Appearance: Colorless liquid.

$R_{f}=0.50\left(100 / 1=\right.$ pentane $\left./ E_{2} O\right)$

${ }^{1} \mathrm{H}-\mathrm{NMR}\left(400 \mathrm{MHz}, \mathrm{CDCl}_{3}\right): \delta=7.40(\mathrm{dt}, J=8.2,1.9 \mathrm{~Hz}, 1 \mathrm{H}), 7.23(\mathrm{dt}, J=7.3,0.9 \mathrm{~Hz}, 1 \mathrm{H}), 7.20(\mathrm{td}, J=$ 7.7, $1.2 \mathrm{~Hz}, 1 \mathrm{H}), 7.12-7.07(\mathrm{~m}, 3 \mathrm{H}), 7.23(\mathrm{dt}, J=7.3,0.9 \mathrm{~Hz}, 1 \mathrm{H}), 7.00(\mathrm{dt}, J=7.6,0.9 \mathrm{~Hz}, 1 \mathrm{H}), 2.32(\mathrm{~s}$, $3 \mathrm{H}), 1.09(\mathrm{~s}, 9 \mathrm{H})$.

${ }^{13} \mathrm{C}-\mathrm{NMR}\left(100 \mathrm{MHz}, \mathrm{CDCl}_{3}\right): \delta=145.2,140.1,137.1,136.3,129.4,128.7,127.3,123.6,123.1,121.6$, 81.1, 38.1, 26.8, 21.0.

IR (ATR): $\tilde{v}\left[\mathrm{~cm}^{-1}\right]=2968,2868,1511,1442,1361$.

HRMS (El, pos., 70eV) m/z:

$\mathrm{M}^{+\bullet}$ Calcd. for $\mathrm{C}_{18} \mathrm{H}_{20} \mathrm{~S}$

268.1286;

Found

268.1307. 


\subsubsection{8-Benzyl-8-(tert-butyl)-7-thiabicyclo[4.2.0]octa-1,3,5-triene (9e)}<smiles>CC(C)(C)C1(Br)Sc2ccccc21</smiles>

Yield: $36.0 \mathrm{mg}, 67 \%$.

Appearance: Colorless liquid.

$\mathrm{R}_{\mathrm{f}}=0.20\left(100 / 1=\right.$ pentane/Et $\left.{ }_{2} \mathrm{O}\right)$

${ }^{1} \mathrm{H}-\mathrm{NMR}\left(400 \mathrm{MHz}, \mathrm{CDCl}_{3}\right): \delta=7.40(\mathrm{dd}, J=8.1,1.3 \mathrm{~Hz}, 1 \mathrm{H}), 7.16-7.08(\mathrm{~m}, 4 \mathrm{H}), 7.05-6.97(\mathrm{~m}, 2 \mathrm{H})$, 6.95-6.92 (m, $2 \mathrm{H}), 3.57(\mathrm{~d}, J=13.8 \mathrm{~Hz}, 1 \mathrm{H}), 3.10(\mathrm{~d}, J=13.8 \mathrm{~Hz}, 1 \mathrm{H}), 1.10(\mathrm{~s}, 9 \mathrm{H})$.

${ }^{13} \mathrm{C}-\mathrm{NMR}\left(100 \mathrm{MHz}_{\mathrm{CDCl}}\right): \delta=138.8,136.4,134.1,132.7,130.8,130.6,128.4,127.0,126.8,123.3$, $83.8,41.7,41.1,26.0$.

IR (ATR): $\tilde{v}\left[\mathrm{~cm}^{-1}\right]=2956,1729,1458,1425,1244,1202$.

HRMS (El, pos., 70eV) m/z: $\quad M^{+\bullet}$ Calcd. for $\mathrm{C}_{18} \mathrm{H}_{20} \mathrm{~S}$

268.1286;

Found

268.1278. 


\subsubsection{8-(tert-Butyl)-8-(4-chlorophenyl)-7-thiabicyclo[4.2.0]octa-1,3,5-triene (9f)}

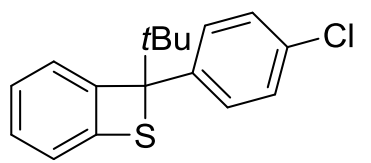

Yield: $39.9 \mathrm{mg}, 69 \%$.

Appearance: Colorless liquid.

$R_{f}=0.65\left(100 / 1=\right.$ pentane $\left./ \mathrm{Et}_{2} \mathrm{O}\right)$

${ }^{1} \mathrm{H}-\mathrm{NMR}\left(500 \mathrm{MHz}, \mathrm{CDCl}_{3}\right): \delta=7.46-7.44(\mathrm{~m}, 2 \mathrm{H}), 7.26-7.24(\mathrm{~m}, 2 \mathrm{H}), 7.22-7.21(\mathrm{~m}, 2 \mathrm{H}), 7.14-7.11(\mathrm{~m}$, $1 \mathrm{H}), 7.03-7.01(\mathrm{~m}, 1 \mathrm{H}), 1.08(\mathrm{~s}, 9 \mathrm{H})$.

Note: Arene signal overlaps inseparably with $\mathrm{CDCl}_{3}$ signal creating a small error in the respective integral.

${ }^{13} \mathrm{C}-\mathrm{NMR}\left(125 \mathrm{MHz}, \mathrm{CDCl}_{3}\right): \delta=144.7,139.8,138.7,132.6,130.8,129.1,126.7,123.9,123.0,121.7$, 80.2, 38.0, 26.7.

IR (ATR): $\tilde{v}\left[\mathrm{~cm}^{-1}\right]=2968,1487,1443,1393,1362,1261,1221,1092$.

HRMS (El, pos., 70eV) m/z:

$\mathrm{M}^{+\bullet}$ Calcd. for $\mathrm{C}_{17} \mathrm{H}_{17} \mathrm{ClS}$

288.0740;

Found

288.0741. 


\subsubsection{8-(tert-Butyl)-8-(thien-2-yl)-7-thiabicyclo[4.2.0]octa-1,3,5-triene (9g)}

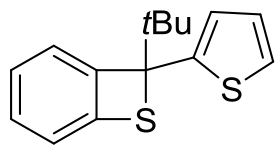

Yield: $39.1 \mathrm{mg}, 75 \%$.

Appearance: Colorless needles.

$\mathrm{R}_{\mathrm{f}}=0.45\left(100 / 1=\right.$ pentane $\left./ \mathrm{Et}_{2} \mathrm{O}\right)$

${ }^{1} \mathrm{H}-\mathrm{NMR}\left(500 \mathrm{MHz}, \mathrm{CDCl}_{3}\right.$ ): $\delta=7.23$ (ddd, $J=7.8,6.8,2.3 \mathrm{~Hz}, 1 \mathrm{H}$ ), 7.17 (dd, $J=5.1,1.3 \mathrm{~Hz}, 1 \mathrm{H}$ ), $7.15-$ $7.10(\mathrm{~m}, 2 \mathrm{H}), 7.06(\mathrm{dd}, J=3.7,1.2 \mathrm{~Hz}, 1 \mathrm{H}), 7.04(\mathrm{dt}, J=7.6,0.9 \mathrm{~Hz}, 1 \mathrm{H}), 6.88(\mathrm{dd}, J=5.2,3.7 \mathrm{~Hz}, 1 \mathrm{H})$, $1.18(\mathrm{~s}, 9 \mathrm{H})$.

${ }^{13} \mathrm{C}-\mathrm{NMR}\left(125 \mathrm{MHz}, \mathrm{CDCl}_{3}\right): \delta=145.0,144.3,139.7,129.1,128.1,125.5,124.3,123.9,122.4,122.0$, $76.9,38.2,26.9$.

IR (ATR): $\tilde{v}\left[\mathrm{~cm}^{-1}\right]=2967,2927,2898,1571,1438,1359,1222,1046,830,776,734,692$.

$\mathrm{mp}=72-74^{\circ} \mathrm{C}$

HRMS (EI, pos., 70eV) m/z:

$\mathrm{M}^{+\bullet}$ Calcd. for $\mathrm{C}_{15} \mathrm{H}_{16} \mathrm{~S}_{2}$

260.0693;

Found

260.0687 . 


\subsubsection{8-(tert-Butyl)-4-methyl-8-phenyl-7-thiabicyclo[4.2.0]octa-1,3,5-triene (10a)}

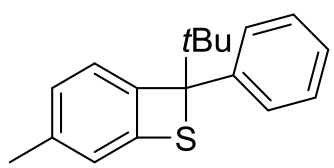

Yield: $33.2 \mathrm{mg}, 62 \%$.

Appearance: Colorless liquid.

$R_{f}=0.50\left(200 / 1=\right.$ pentane $\left./ E t_{2} O\right)$

${ }^{1} \mathrm{H}-\mathrm{NMR}\left(500 \mathrm{MHz}, \mathrm{CDCl}_{3}\right): \delta=7.51-7.49(\mathrm{~m}, 2 \mathrm{H}), 7.28-7.25(\mathrm{~m}, 2 \mathrm{H}), 7.22-7.19(\mathrm{~m}, 1 \mathrm{H}), 7.13(\mathrm{~d}, J=$ $7.26 \mathrm{~Hz}, 1 \mathrm{H}), 6.94-6.92(\mathrm{~m}, 1 \mathrm{H}), 6.85-6.84(\mathrm{~m}, 1 \mathrm{H}), 2.30(\mathrm{~s}, 3 \mathrm{H}), 1.09(\mathrm{~s}, 9 \mathrm{H})$.

${ }^{13} \mathrm{C}-\mathrm{NMR}\left(125 \mathrm{MHz}, \mathrm{CDCl}_{3}\right): \delta=142.0,140.4,139.6,138.9,129.5,126.5,126.5,124.5,122.9,122.2$, $80.9,38.0,26.8,22.0$.

IR (ATR): $\tilde{v}\left[\mathrm{~cm}^{-1}\right]=2968,1597,1575,1445,1393,1361,1307,1169$

HRMS (El, pos., 70eV) m/z:

$\mathrm{M}^{+\bullet}$ Calcd. for $\mathrm{C}_{18} \mathrm{H}_{20} \mathrm{~S}$

268.1286;

Found

268.1280. 
<smiles>Cc1ccc2c(c1)SC2(c1cccs1)C(C)(C)C</smiles>

Yield: $42.8 \mathrm{mg}, 78 \%$.

Appearance: Colorless solid.

$\mathrm{R}_{\mathrm{f}}=0.50\left(200 / 1=\right.$ pentane $\left./ \mathrm{Et}_{2} \mathrm{O}\right)$

${ }^{1} \mathrm{H}-\mathrm{NMR}\left(500 \mathrm{MHz}, \mathrm{CDCl}_{3}\right): \delta=7.15(\mathrm{dd}, J=5.1 \mathrm{~Hz}, 1.26 \mathrm{~Hz}, 1 \mathrm{H}), 7.04(\mathrm{dd}, J=3.60 \mathrm{~Hz}, 1.2 \mathrm{~Hz}, 1 \mathrm{H}), 7.20$ (d, J = 7.6 Hz, $1 \mathrm{H})$, 6-94-6.92 (m, $1 \mathrm{H}), 6.89-6.87(\mathrm{~m}, 2 \mathrm{H}), 2.32(\mathrm{~s}, 3 \mathrm{H}), 1.17(\mathrm{~s}, 9 \mathrm{H})$.

${ }^{13} \mathrm{C}-\mathrm{NMR}\left(125 \mathrm{MHz}, \mathrm{CDCl}_{3}\right): \delta=144.7,141.9,139.2,128.0,125.5,124.7,124.2,122.6,122.1,76.6,38.2$, $26.9,22.1$. (One quaternary ring-carbon was not observable above the signal-to-noise ratio).

IR (ATR): $\tilde{v}\left[\mathrm{~cm}^{-1}\right]=2970,1577,1468,1392,1225,1143,1037$.

$\mathrm{mp}=65-67^{\circ} \mathrm{C}$

HRMS (El, pos., 70eV) m/z:

$\mathrm{M}^{+\bullet}$ Calcd. for $\mathrm{C}_{16} \mathrm{H}_{18} \mathrm{~S}_{2}$ 274.0850;

Found 274.0848 . 


\subsubsection{8-(tert-Butyl)-8-phenyl-4-(trifluoromethyl)-7-thiabicyclo[4.2.0]octa-1,3,5-triene}

(11a)<smiles>CC1(c2ccccc2)Sc2cc(C(F)(F)F)ccc21</smiles>

Yield: $39.3 \mathrm{mg}, 61 \%$.

Appearance: Colorless liquid.

$\mathrm{R}_{\mathrm{f}}=0.50\left(200 / 1=\right.$ pentane $\left./ \mathrm{Et}_{2} \mathrm{O}\right)$

${ }^{1} \mathrm{H}-\mathrm{NMR}\left(500 \mathrm{MHz}, \mathrm{CDCl}_{3}\right): \delta=7.15(\mathrm{dd}, J=5.13 \mathrm{~Hz}, 1.26 \mathrm{~Hz}, 1 \mathrm{H}), 7.04(\mathrm{dd}, J=3.60 \mathrm{~Hz}, 1.24 \mathrm{~Hz}, 1 \mathrm{H}$ ), $7.20(\mathrm{~d}, \mathrm{~J}=7.63 \mathrm{~Hz}, 1 \mathrm{H}), 6-94-6.92(\mathrm{~m}, 1 \mathrm{H}), 6.89-6.87(\mathrm{~m}, 2 \mathrm{H}), 2.32(\mathrm{~s}, 3 \mathrm{H}), 1.17(\mathrm{~s}, 9 \mathrm{H})$.

${ }^{13} \mathrm{C}-\mathrm{NMR}\left(125 \mathrm{MHz}, \mathrm{CDCl}_{3}\right): \delta=148.7(\mathrm{~m}, 1 \mathrm{C}), 141.3,139.0,131.2$ (q, $\left.J=33 \mathrm{~Hz}, 1 \mathrm{C}\right), 129.4,127.0$, $126.8,124.0$ (q, $J=273 \mathrm{~Hz}, 1 \mathrm{C}$ ), 123.0, 121.0 (q, $J=4 \mathrm{~Hz}, 1 \mathrm{C}$ ), 118.4 (q, $J=4 \mathrm{~Hz}, 1 \mathrm{C}), 81.8,38.1$, 26.7.

${ }^{19} \mathrm{~F}-\mathrm{NMR}\left(376 \mathrm{MHz}, \mathrm{CDCl}_{3}\right): \delta=-62.9$.

IR (ATR): $\tilde{v}\left[\mathrm{~cm}^{-1}\right]=2968,1403,1313,1250,1166,1041$.

HRMS (El, pos., 70eV) m/z: $\quad \mathrm{M}^{+\bullet}$ Calcd. for $\mathrm{C}_{18} \mathrm{H}_{17} \mathrm{~F}_{3} \mathrm{~S}$

322.1003;

Found

322.1021. 

(11b)<smiles></smiles>

Yield: $50.6 \mathrm{mg}, 77 \%$.

Appearance: Colorless liquid.

$\mathrm{R}_{\mathrm{f}}=0.50\left(200 / 1=\right.$ pentane $\left./ \mathrm{Et}_{2} \mathrm{O}\right)$

${ }^{1} \mathrm{H}-\mathrm{NMR}\left(500 \mathrm{MHz}, \mathrm{CDCl}_{3}\right): \delta=7.41-7.39(\mathrm{~m}, 1 \mathrm{H}), 7.30-7.29(\mathrm{~m}, 1 \mathrm{H}), 7.25-7.23(\mathrm{~m}, 1 \mathrm{H}), 7.20(\mathrm{dd}$, $J=5.10 \mathrm{~Hz}, 1.23 \mathrm{~Hz}, 1 \mathrm{H}), 7.05(\mathrm{dd}, J=3.64 \mathrm{~Hz}, 1.23 \mathrm{~Hz}, 1 \mathrm{H}), 6.90(\mathrm{dd}, J=5.15 \mathrm{~Hz}, 3.64 \mathrm{~Hz}, 1 \mathrm{H}$ ), $1.19(\mathrm{~s}, 9 \mathrm{H})$.

${ }^{13} \mathrm{C}-\mathrm{NMR}\left(125 \mathrm{MHz}, \mathrm{CDCl}_{3}\right): \delta=148.5(\mathrm{~m}, 1 \mathrm{C}), 143.0,140.9,131.5$ (q, $\left.J=32 \mathrm{~Hz}, 1 \mathrm{C}\right), 128.3,125.7$, 124.7, 124.1 (q, $J=274 \mathrm{~Hz}, 1 \mathrm{C}$ ), 122.3, 121.2 (q, $J=4 \mathrm{~Hz}, 1 \mathrm{C}$ ), 118.9 (q, $J=4 \mathrm{~Hz}, 1 \mathrm{C}$ ), 77.7, 38.3, 26.8. ${ }^{19} \mathrm{~F}-\mathrm{NMR}\left(376 \mathrm{MHz}, \mathrm{CDCl}_{3}\right): \delta=-62.9$.

IR (ATR): $\tilde{v}\left[\mathrm{~cm}^{-1}\right]=2969,1469,1402,1309,1247,1165,1121,1071,1042$.

HRMS (El, pos., 70eV) m/z: $\quad M^{+\bullet}$ Calcd. for $\mathrm{C}_{16} \mathrm{H}_{15} \mathrm{~F}_{3} \mathrm{~S}_{2}$ 328.0567;

Found 328.0575. 


\subsection{NMR-Spectra of Thiols and Precursors}

2.8.1 S-(2-Formylphenyl) O-(4-Methoxyphenyl) Carbonothioate (1b)

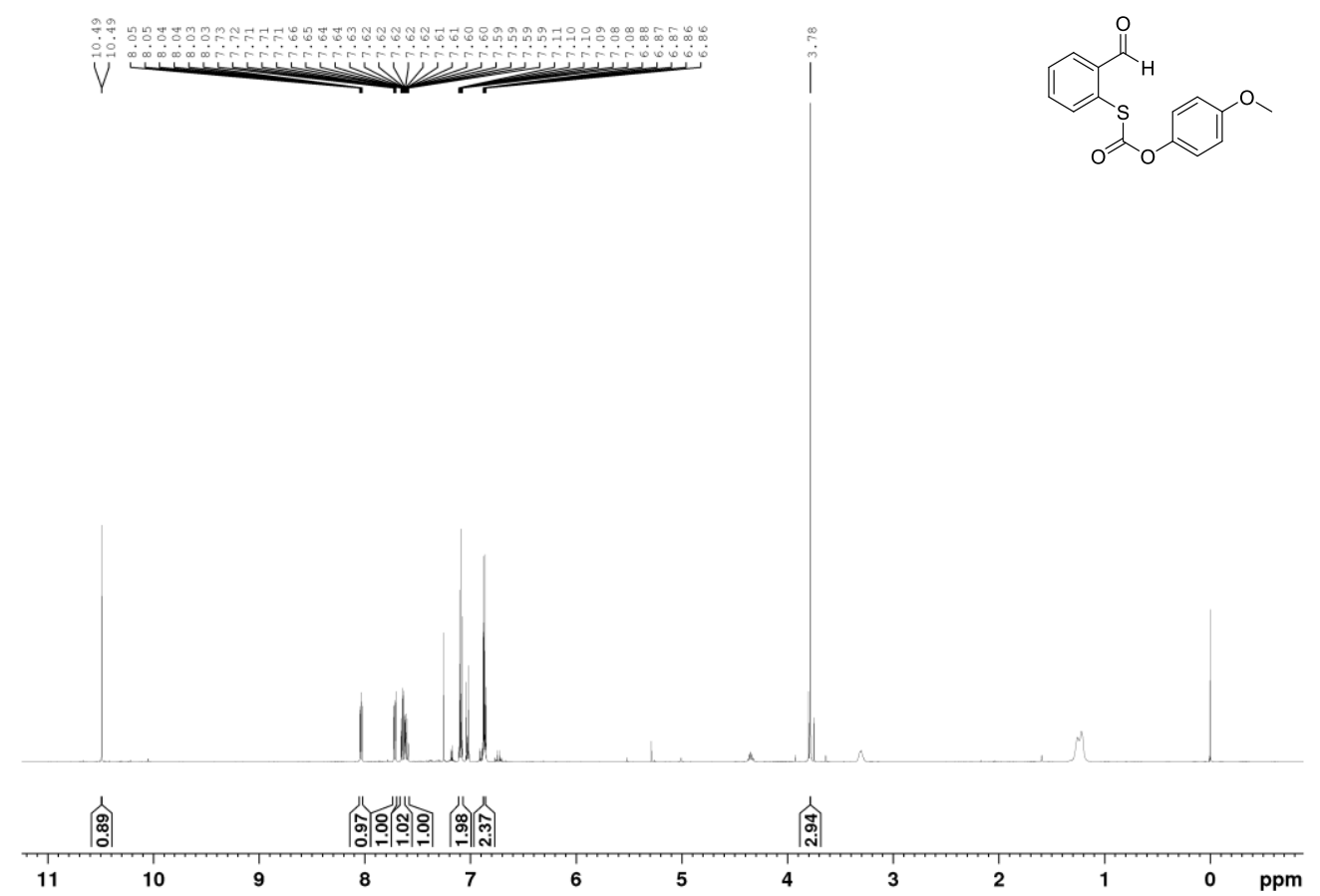

${ }^{1} \mathrm{H}-\mathrm{NMR}\left(500 \mathrm{MHz}, \mathrm{CDCl}_{3}\right)$

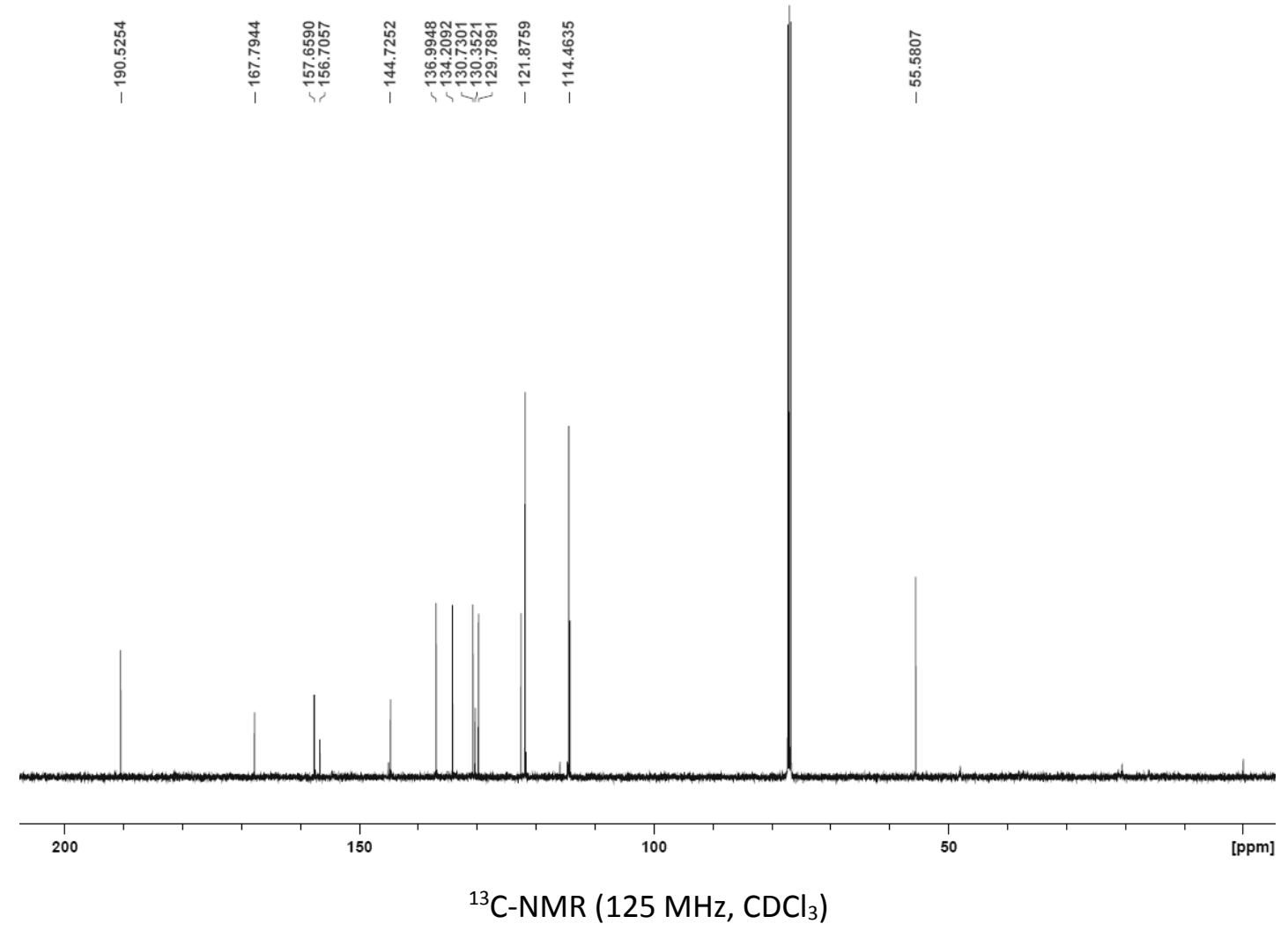


2.8.2 O-Benzyl S-(2-Formylphenyl) Carbonothioate (1c)

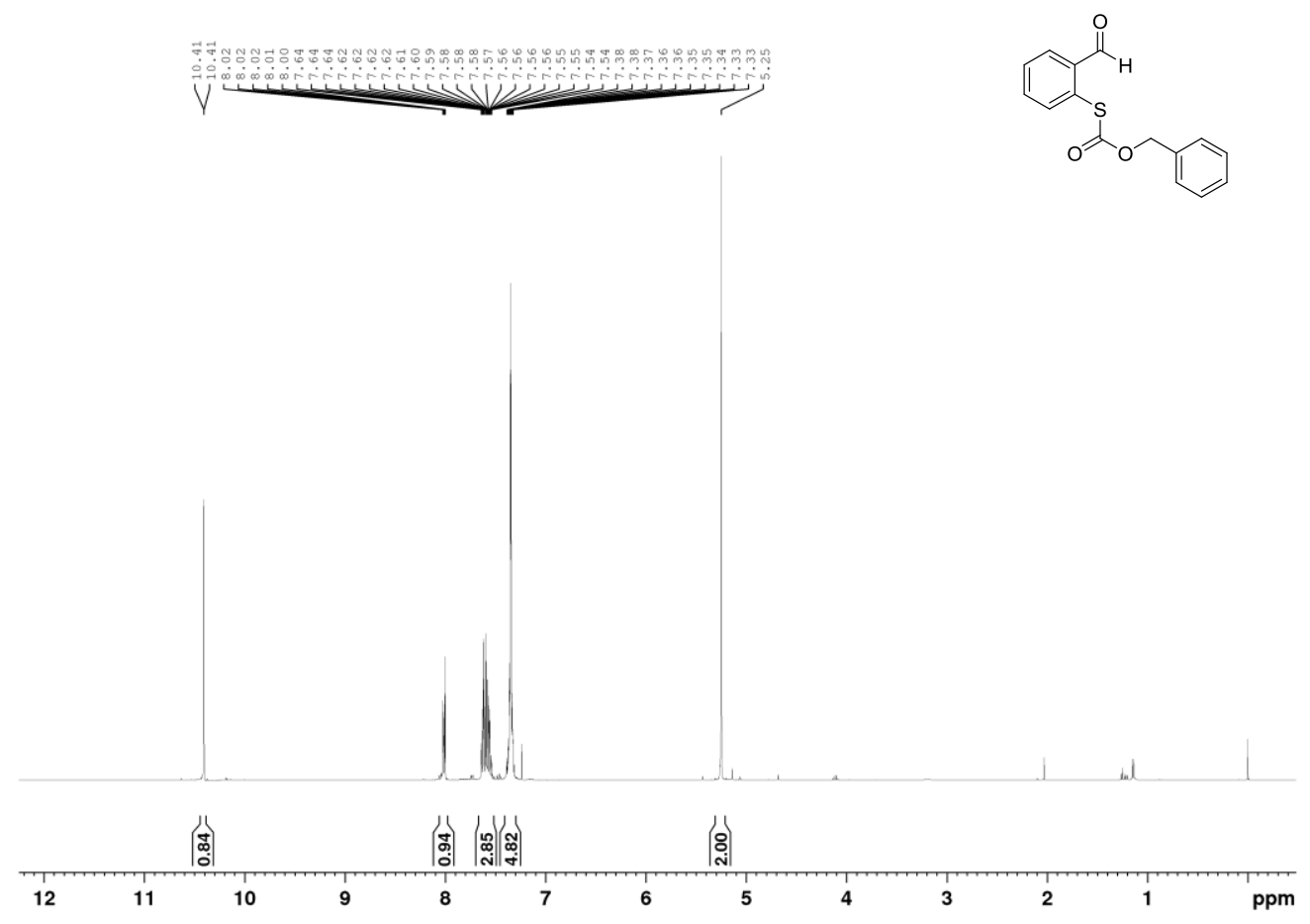

${ }^{1} \mathrm{H}-\mathrm{NMR}\left(500 \mathrm{MHz}, \mathrm{CDCl}_{3}\right)$

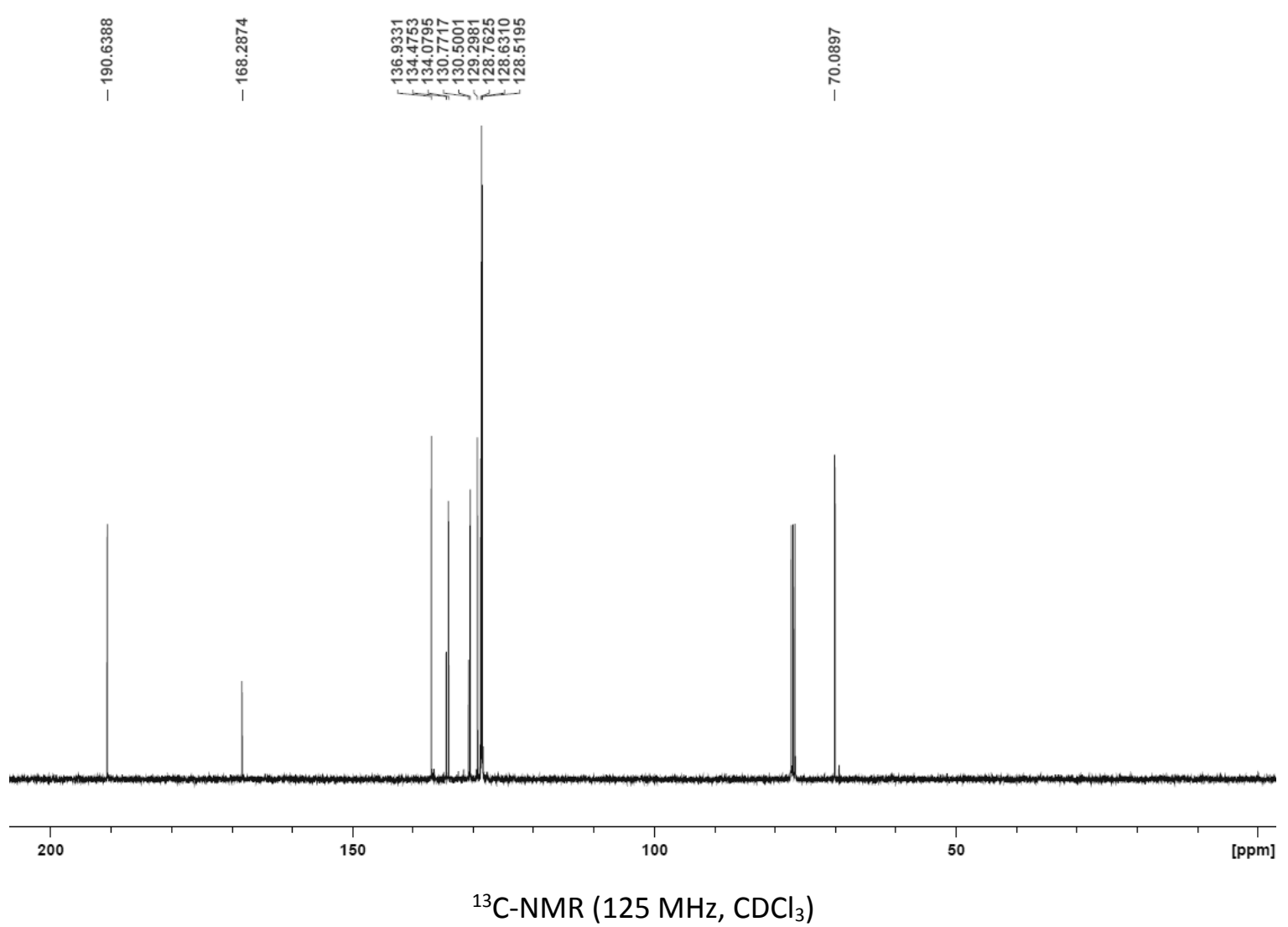


2.8.3 O-Allyl S-(2-Formylphenyl) Carbonothioate (1d)

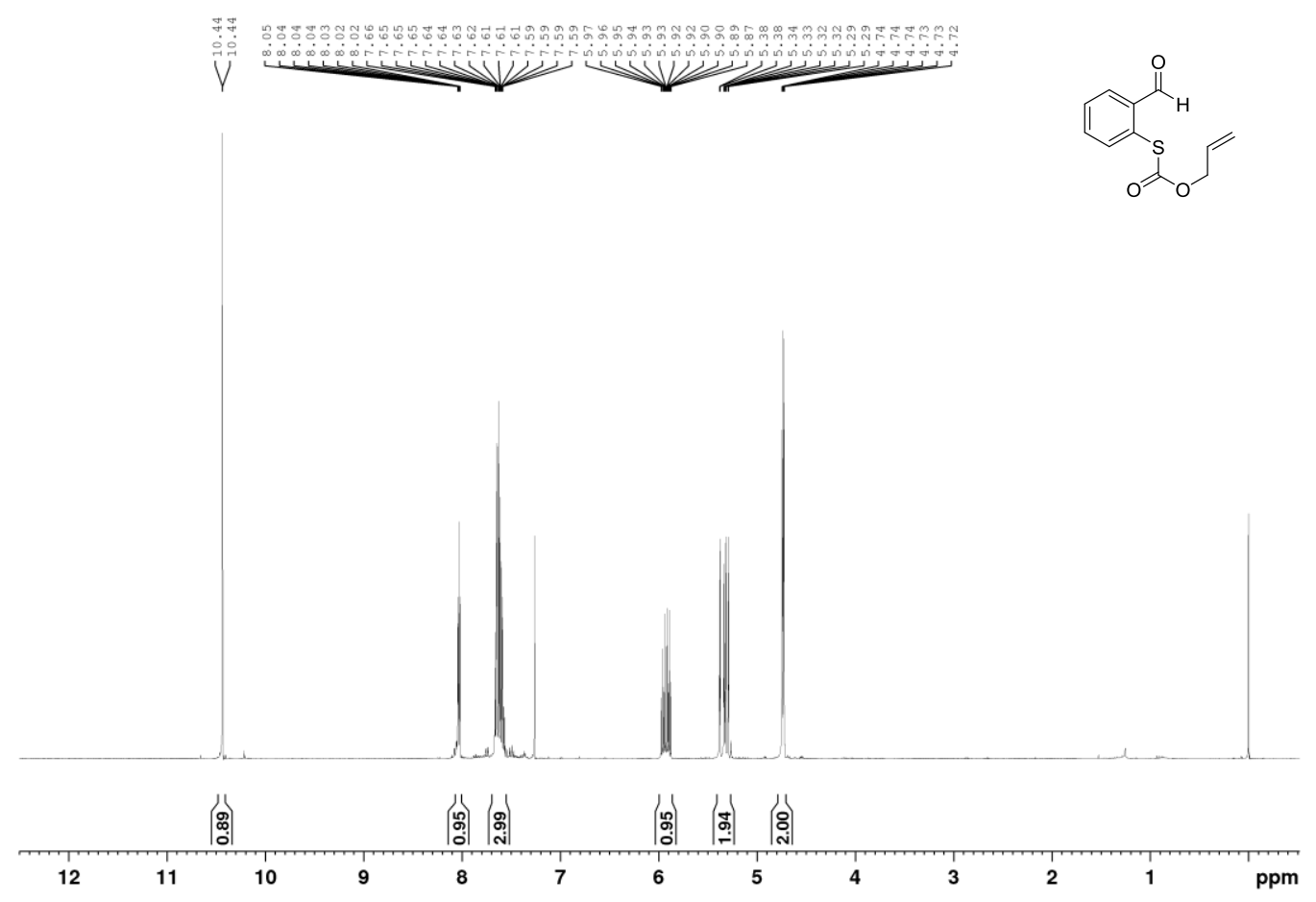

${ }^{1} \mathrm{H}-\mathrm{NMR}\left(500 \mathrm{MHz}, \mathrm{CDCl}_{3}\right)$

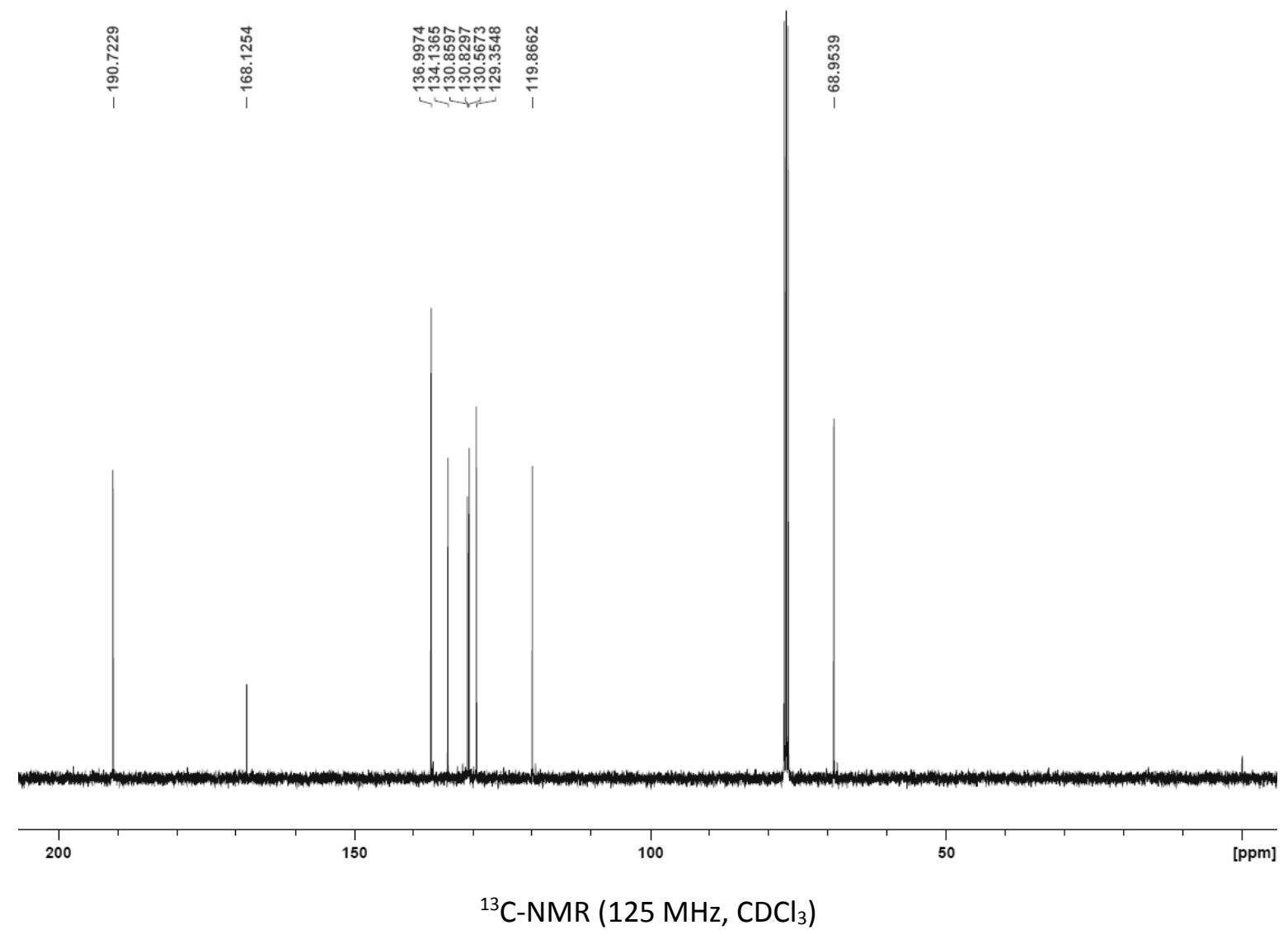


2.8.4 S-(2-Formylphenyl) O-Methyl Carbonothioate (1e)

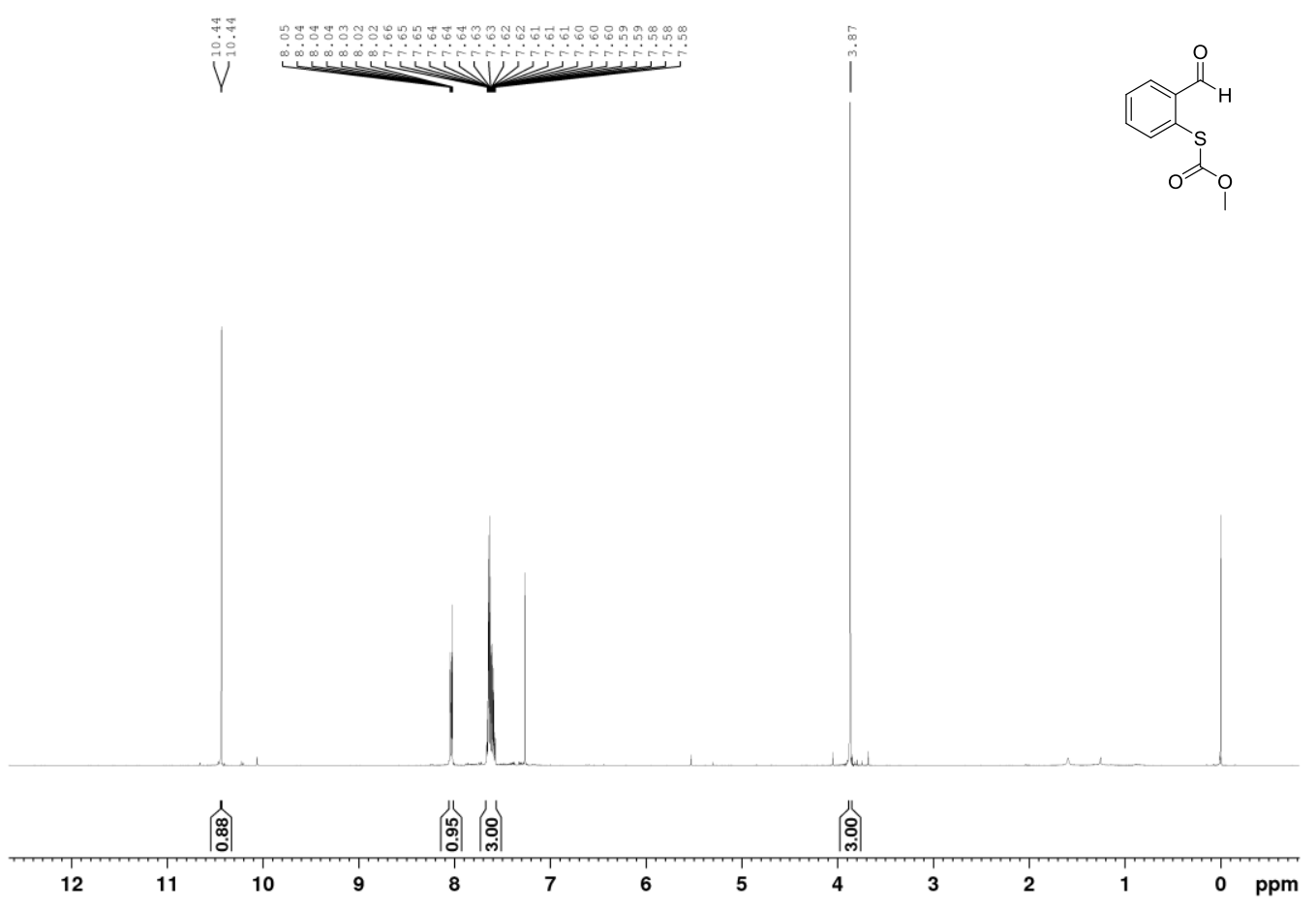

${ }^{1} \mathrm{H}-\mathrm{NMR}\left(500 \mathrm{MHz}, \mathrm{CDCl}_{3}\right)$

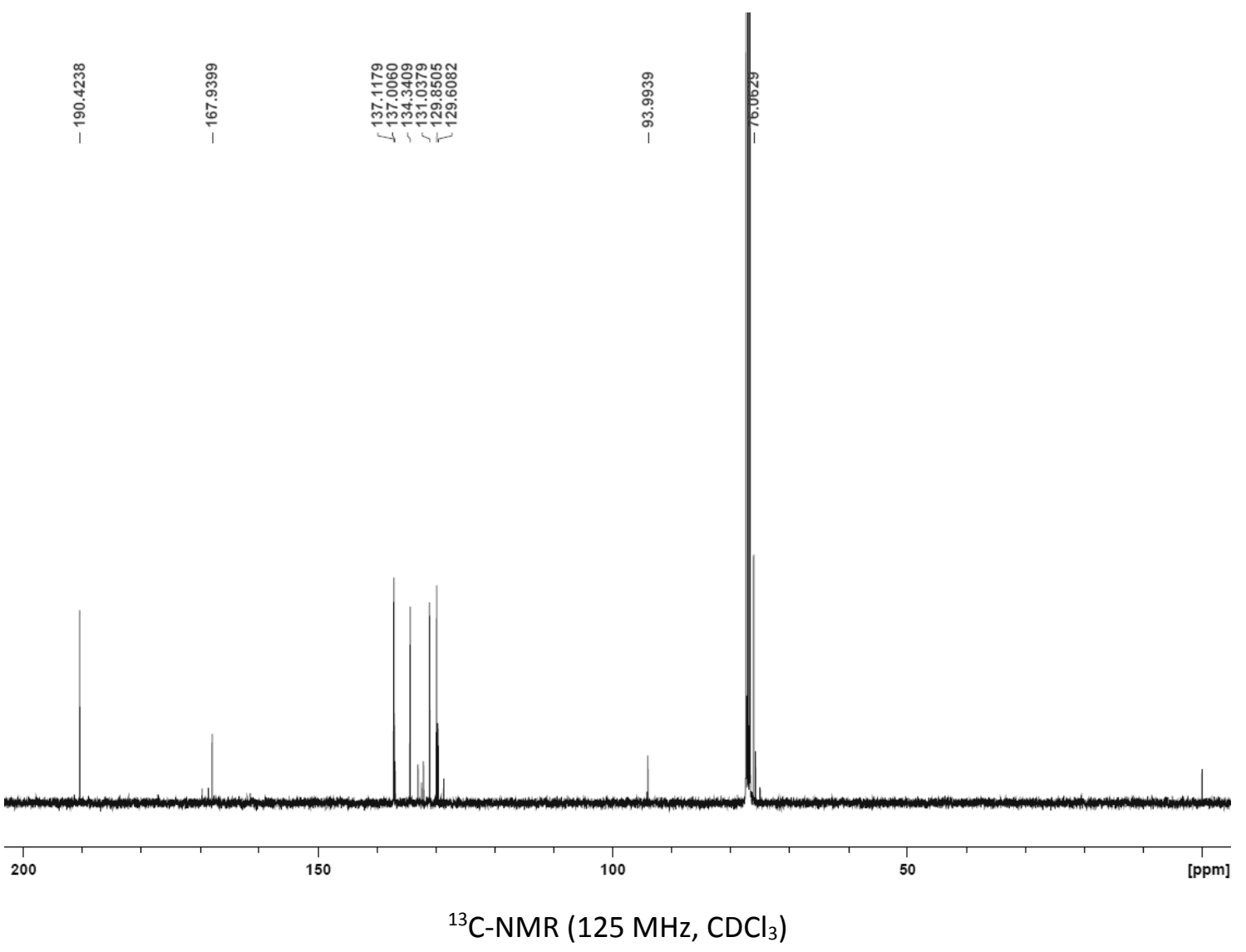


2.8.5 S-(2-Formylphenyl) O-(2,2,2-Trichloroethyl) Carbonothioate (1f)

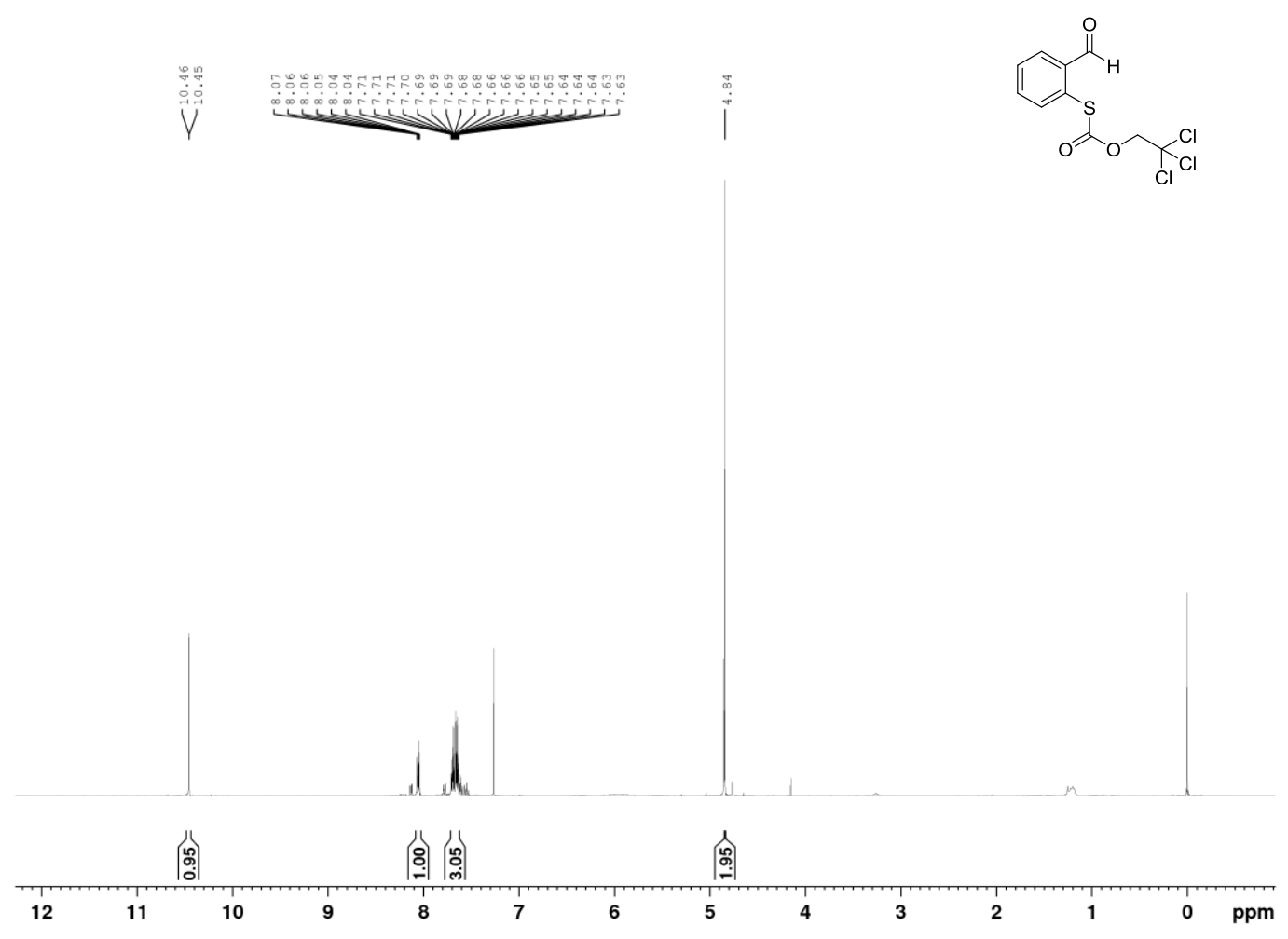

${ }^{1} \mathrm{H}-\mathrm{NMR}\left(400 \mathrm{MHz}, \mathrm{CDCl}_{3}\right)$

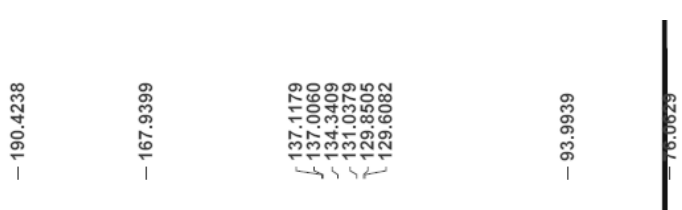

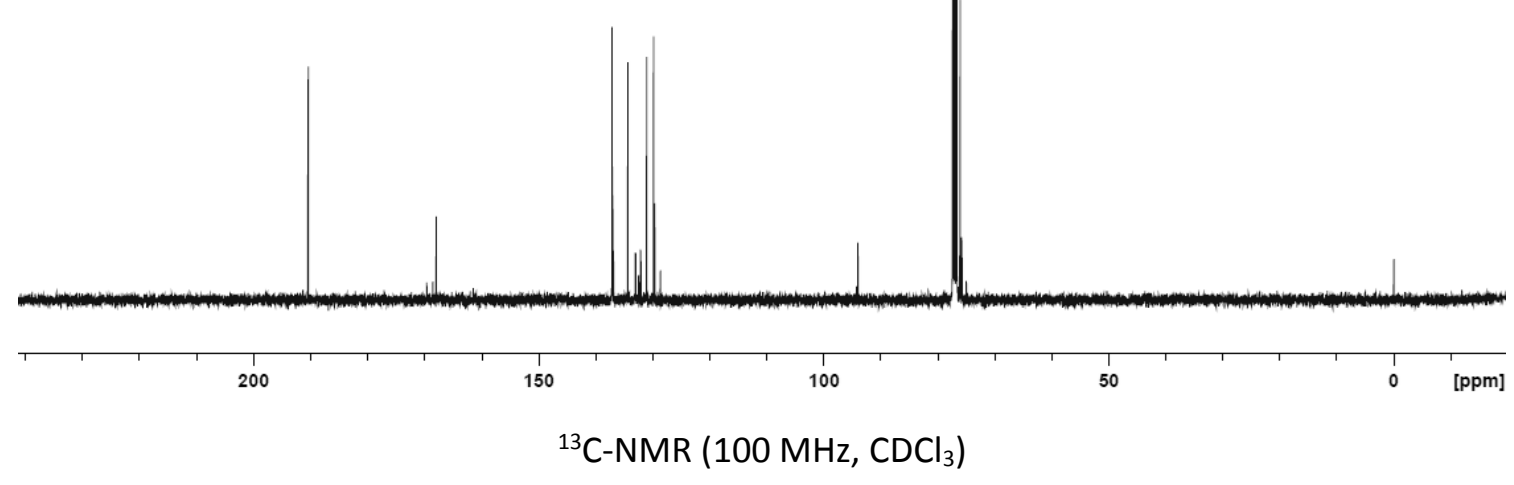




\subsubsection{S-(2-Formylphenyl) O-Phenyl Carbonothioate (1g)}

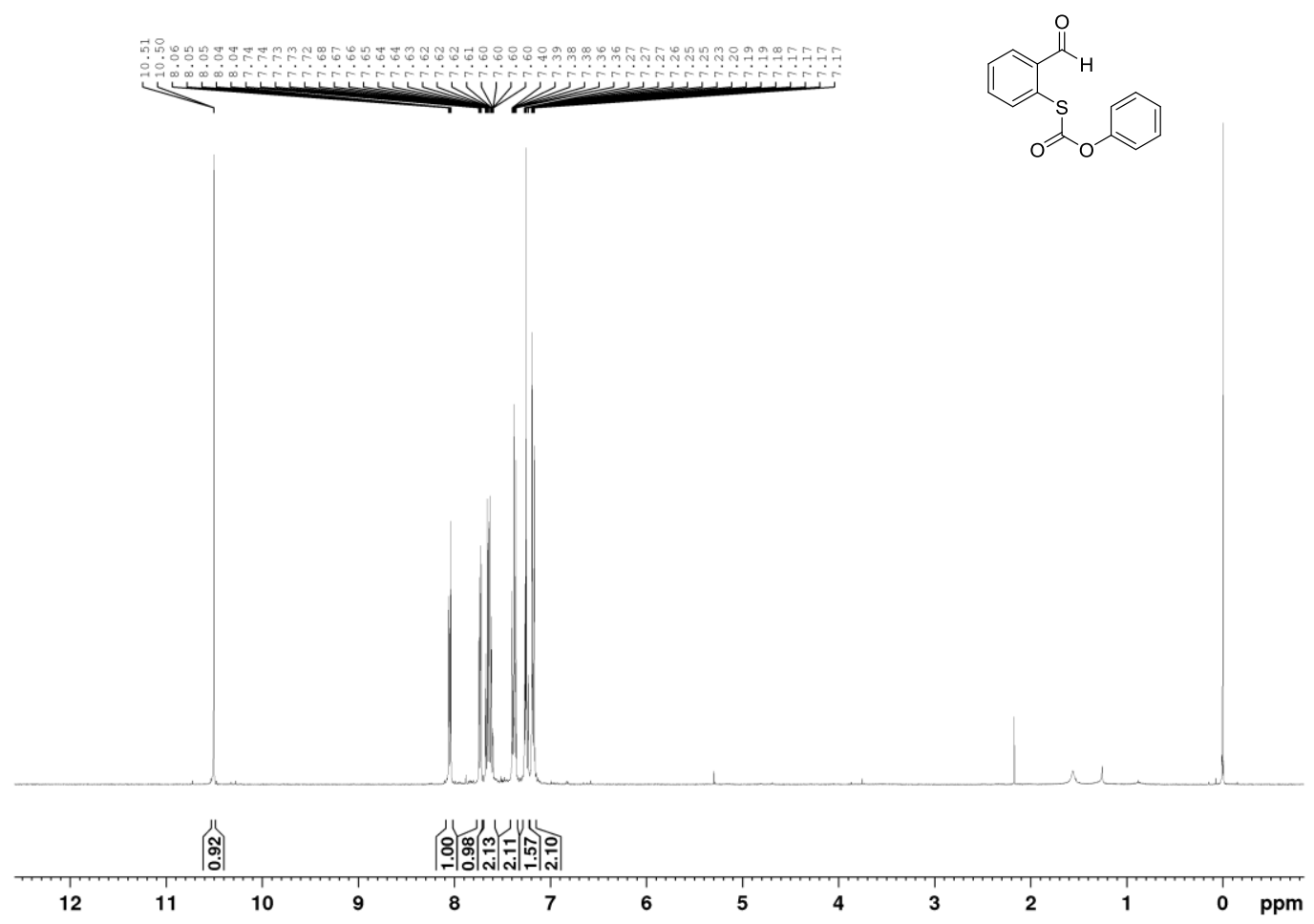

${ }^{1} \mathrm{H}-\mathrm{NMR}\left(400 \mathrm{MHz}, \mathrm{CDCl}_{3}\right)$
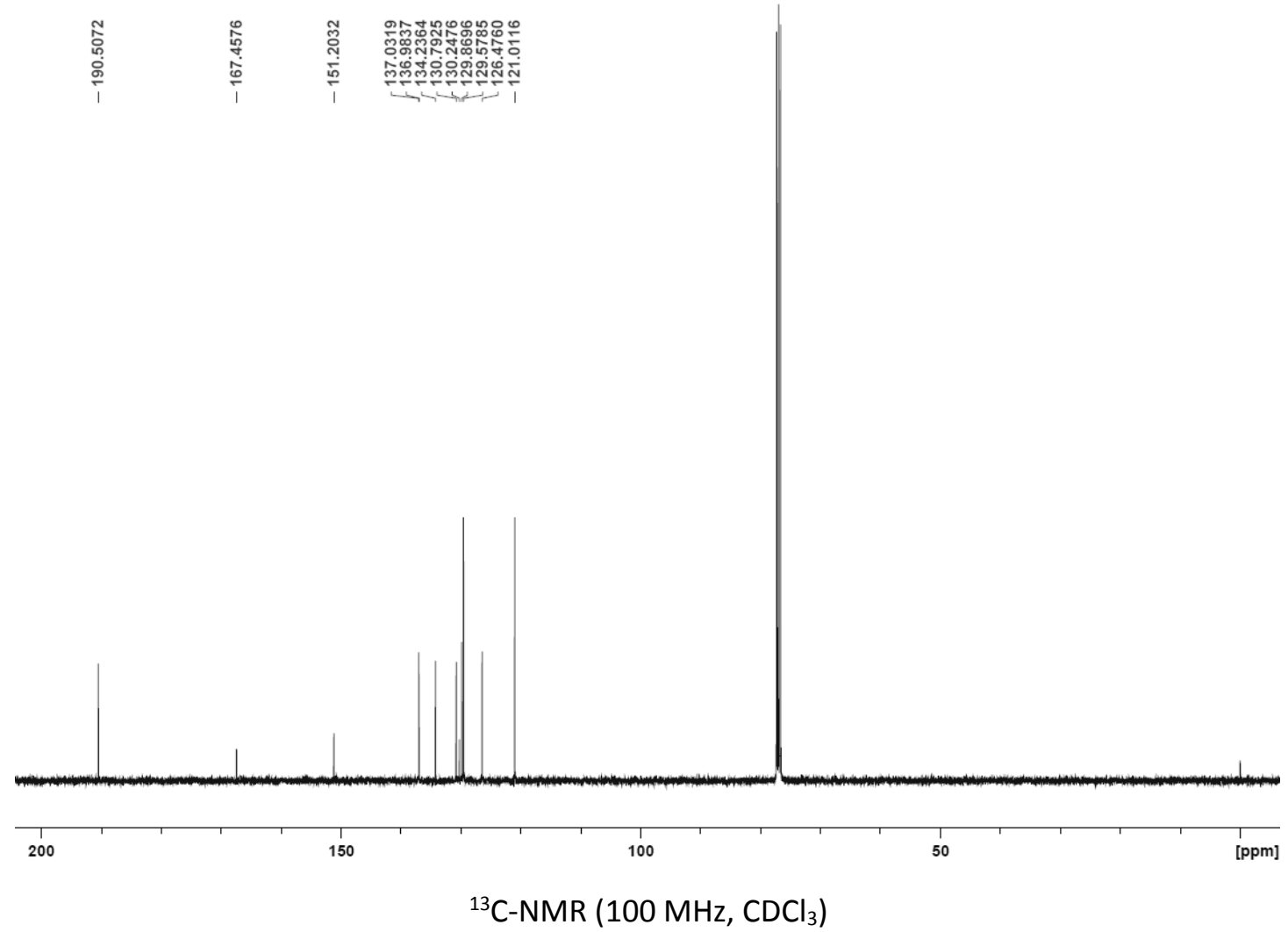
2.8.7 1-(2-Mercapto-4-methylphenyl)-2,2-dimethylpropan-1-one (28)

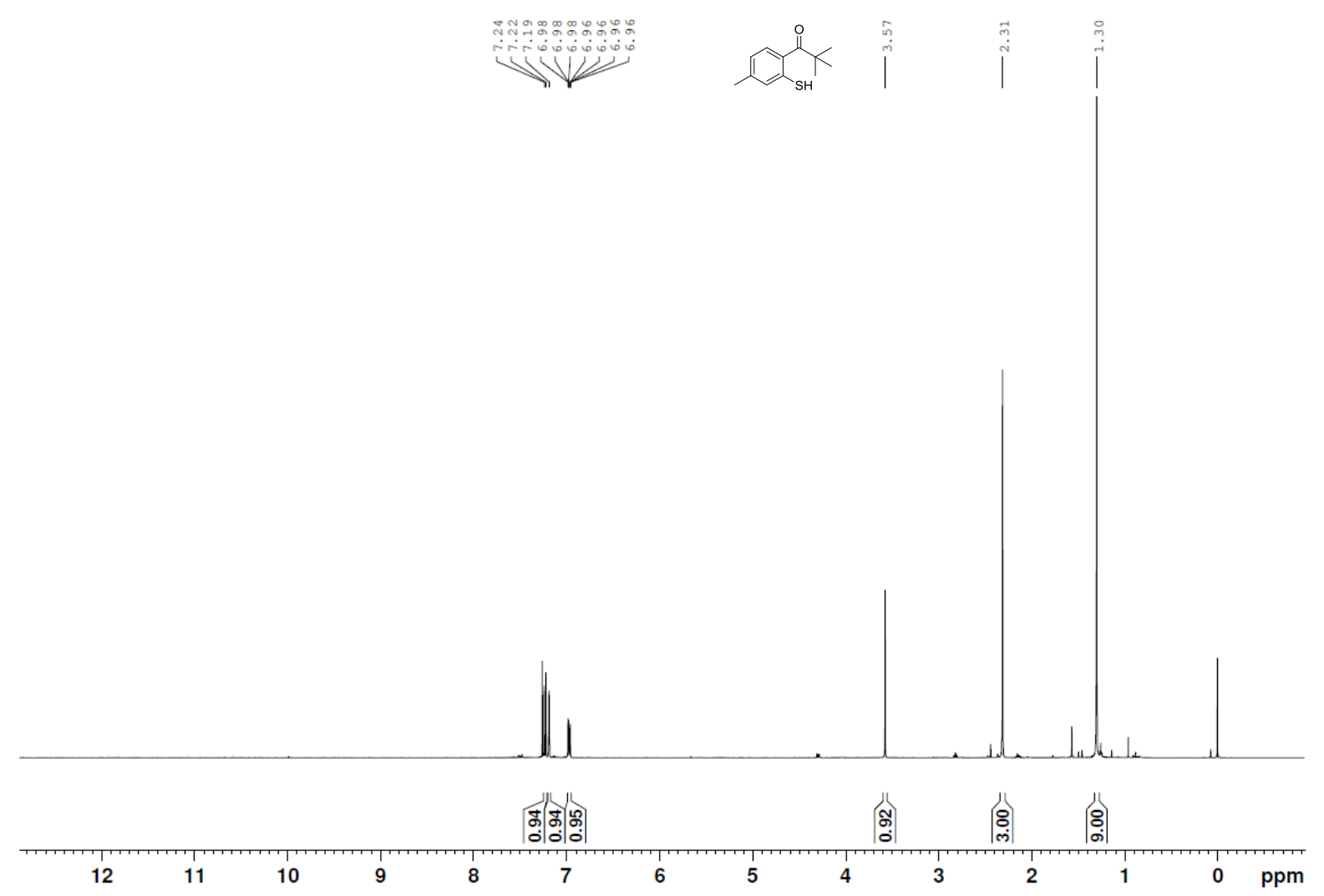

${ }^{1} \mathrm{H}-\mathrm{NMR}\left(400 \mathrm{MHz}, \mathrm{CDCl}_{3}\right)$

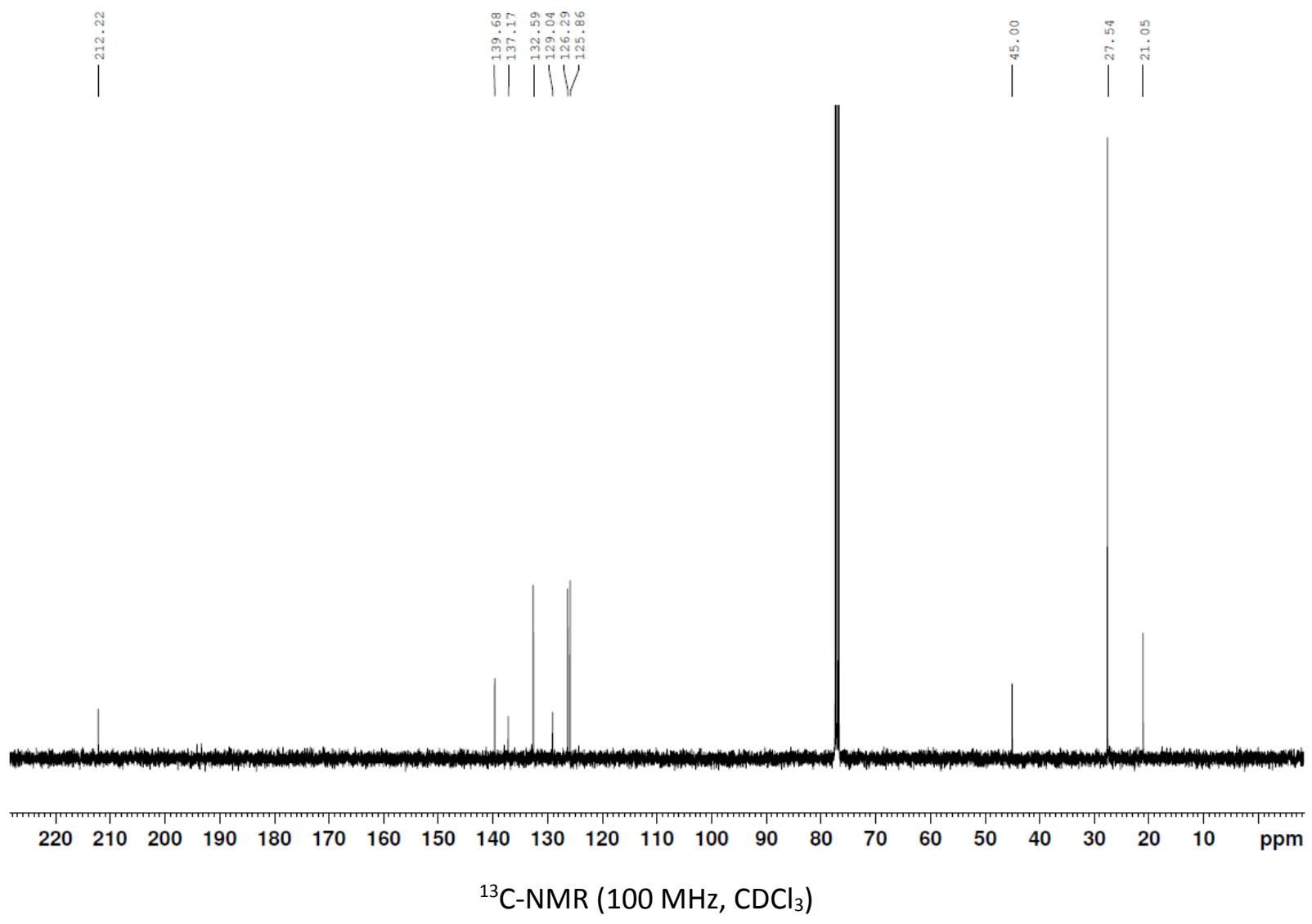


2.8.8 1-(2-mercapto-4-(trifluoromethyl)phenyl)-2,2-dimethylpropan-1-one (29)

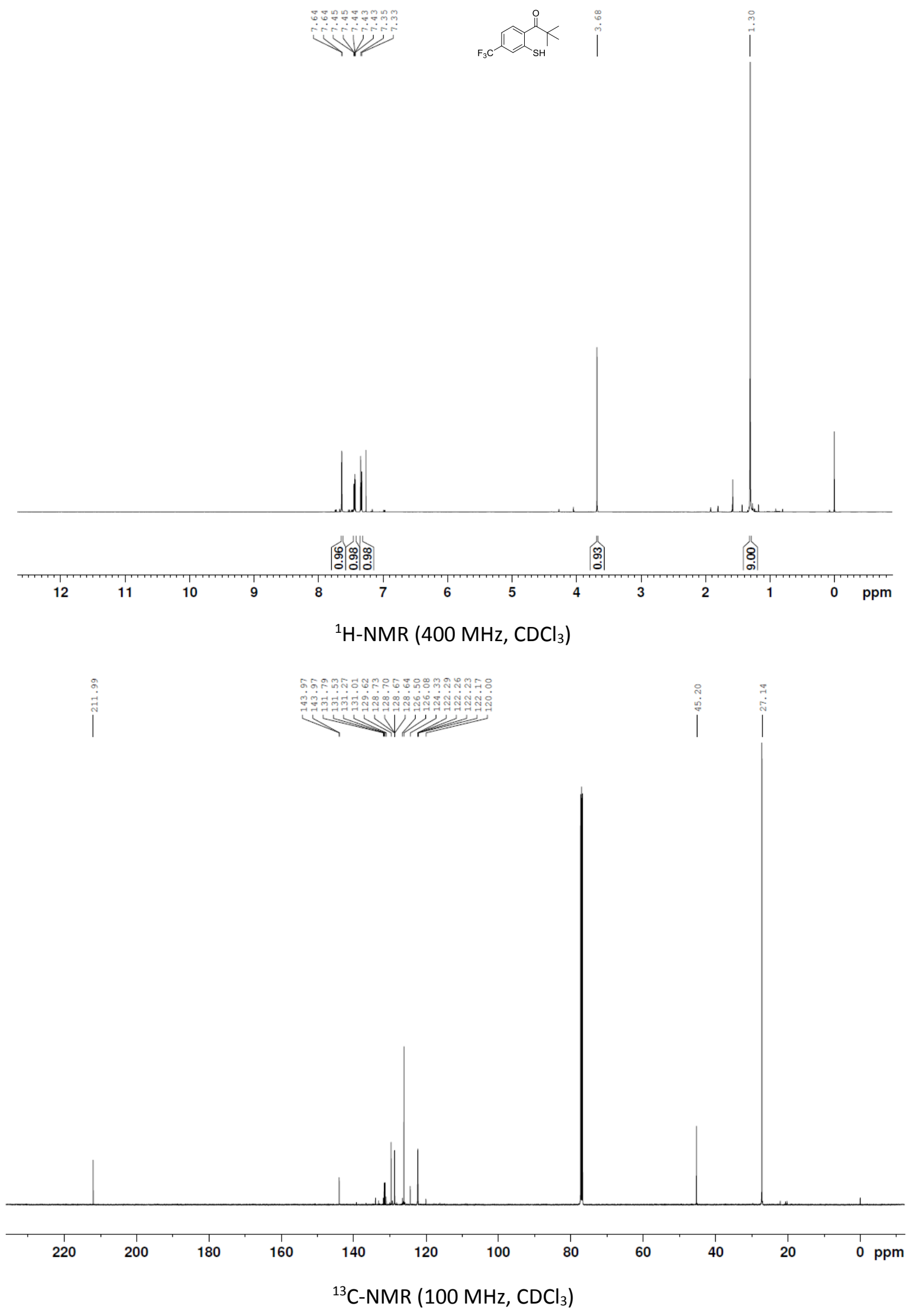




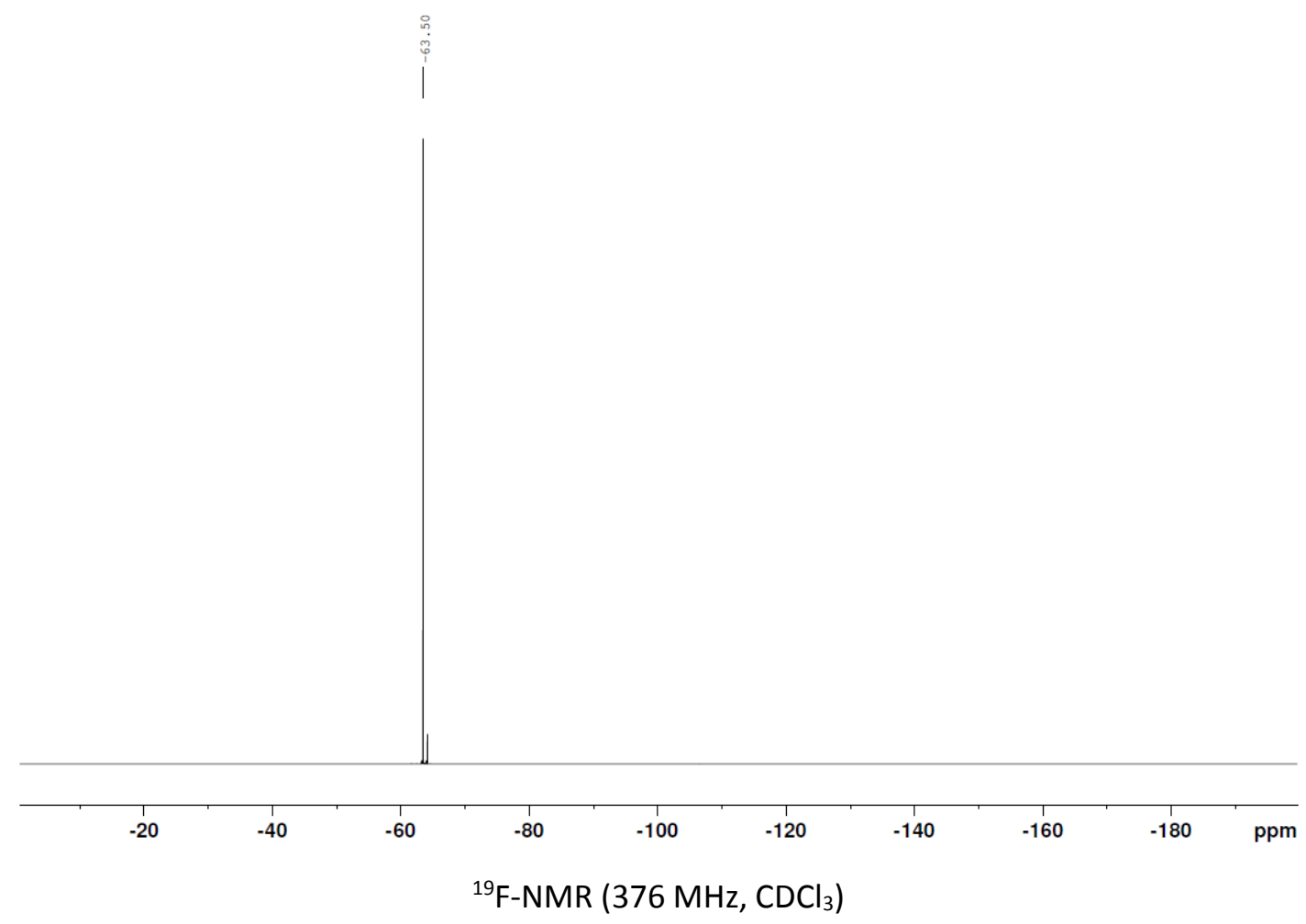




\subsection{NMR-Spectra of Carbamothioates}

2.9.1 S-(2-Benzoylphenyl) Dimethylcarbamothioate (4)
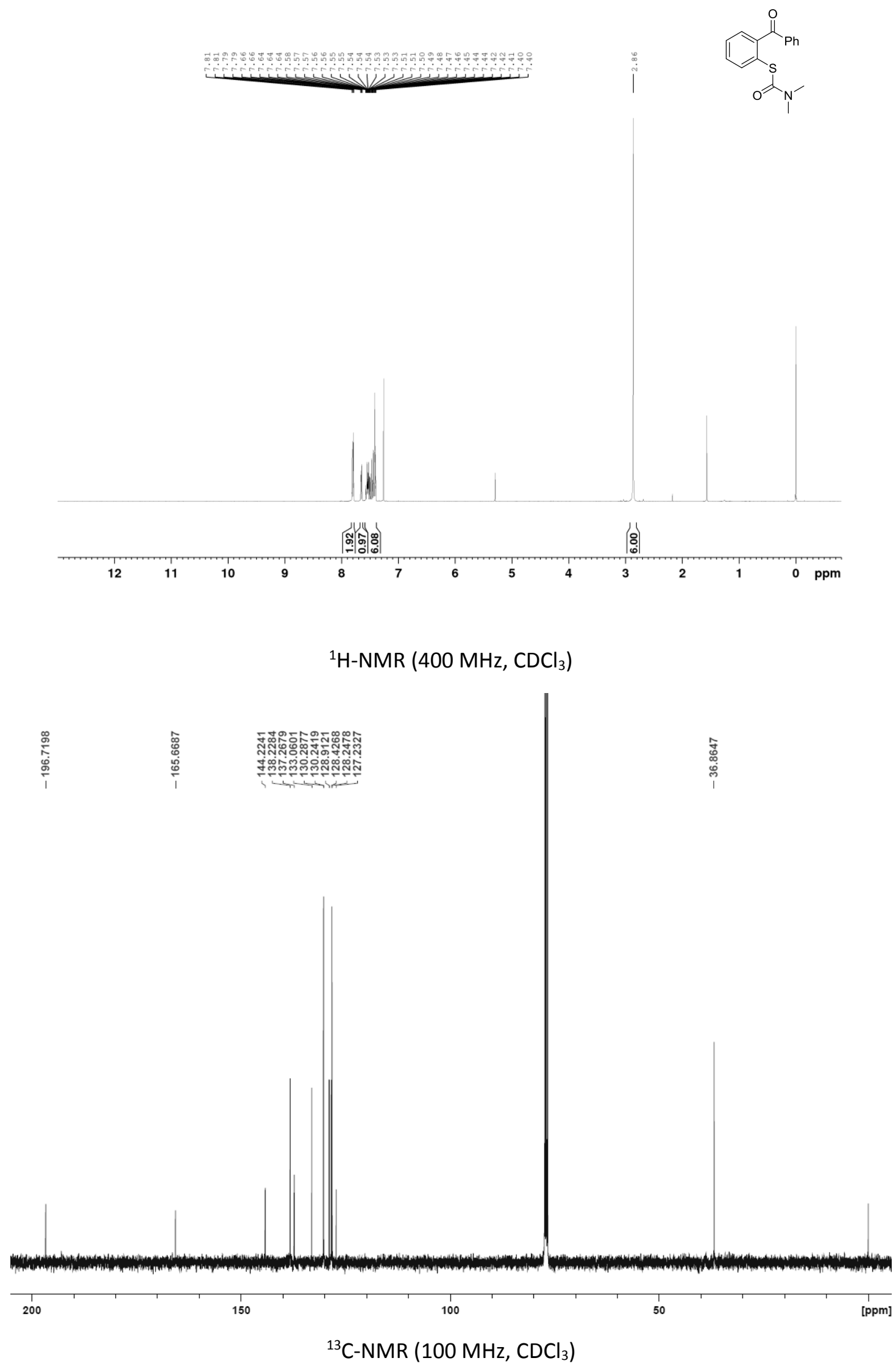
2.9.2 S-(2-Pivaloylphenyl) Dimethylcarbamothioate (5)

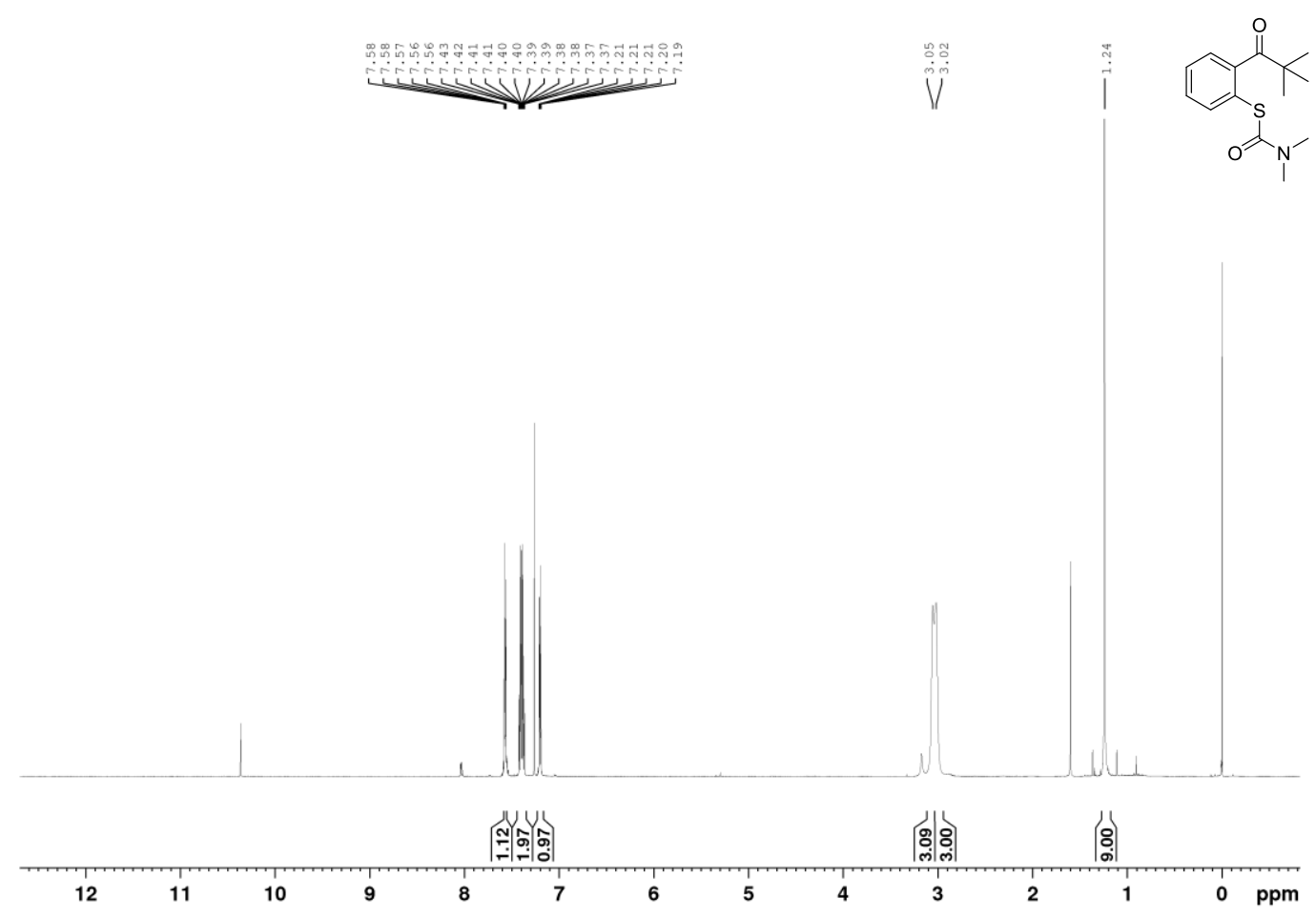

${ }^{1} \mathrm{H}-\mathrm{NMR}\left(500 \mathrm{MHz}, \mathrm{CDCl}_{3}\right)$

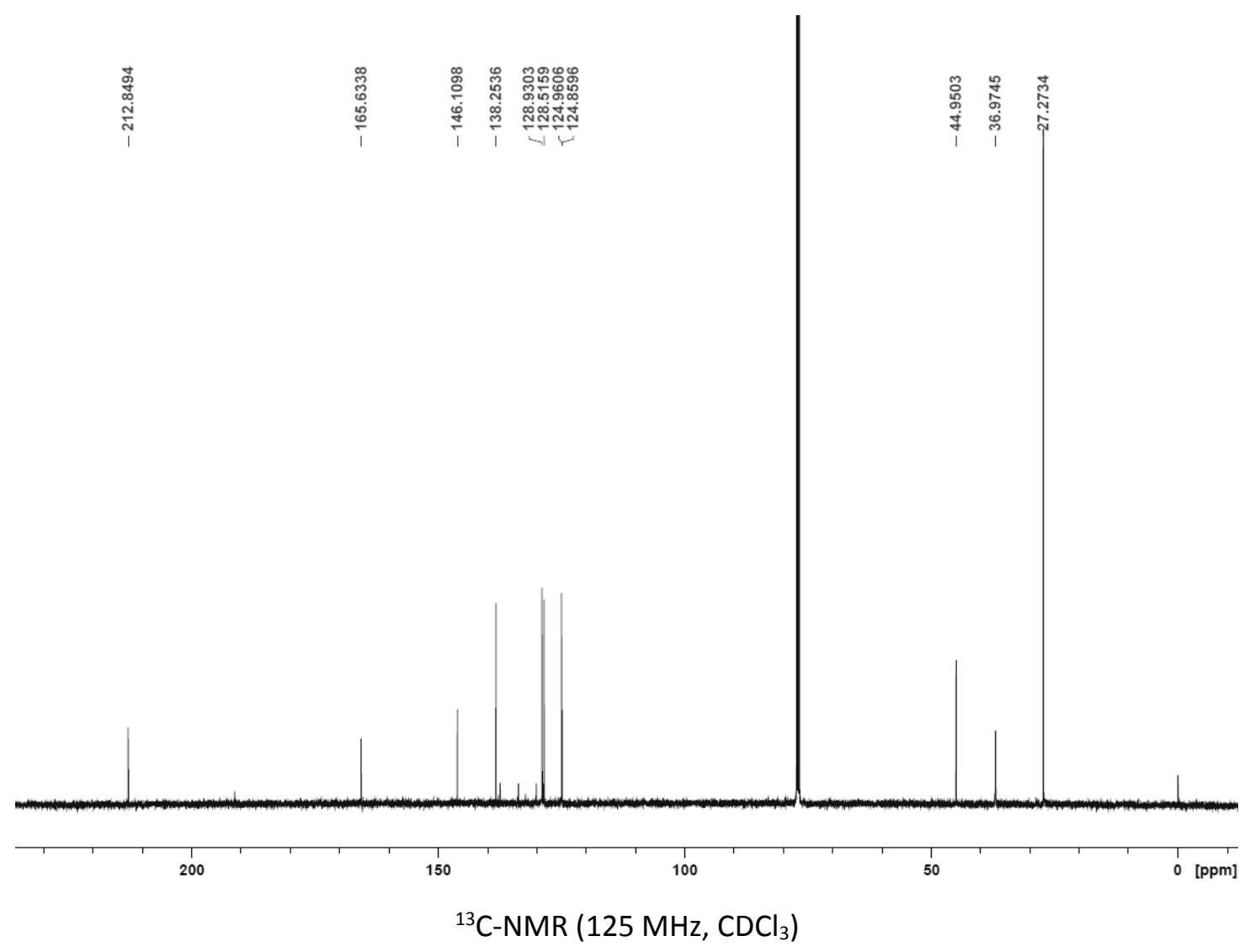


2.9.3 S-(2-Benzoyl-4-methylphenyl) Dimethylcarbamothioate (6)

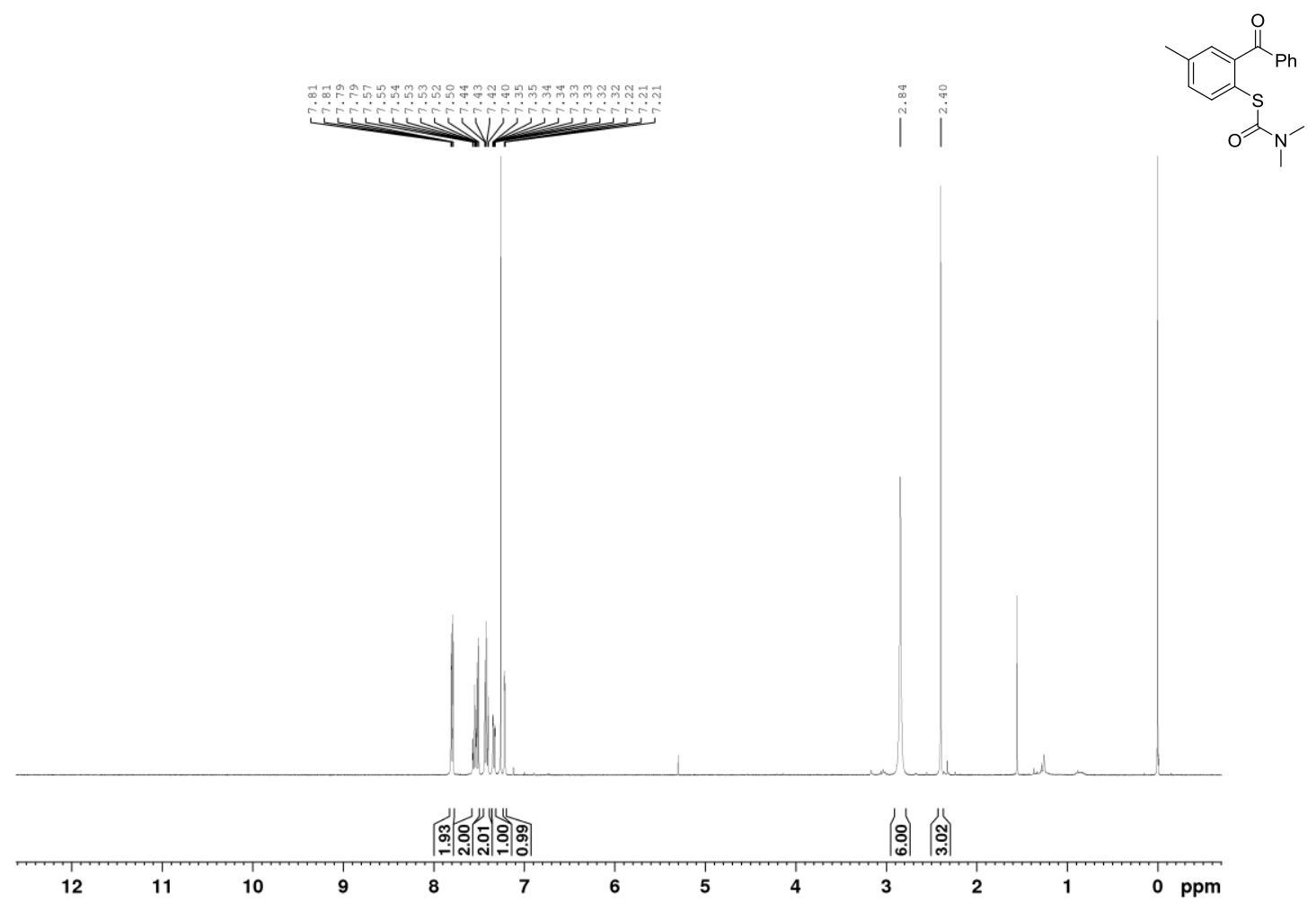

${ }^{1} \mathrm{H}-\mathrm{NMR}\left(400 \mathrm{MHz}, \mathrm{CDCl}_{3}\right)$

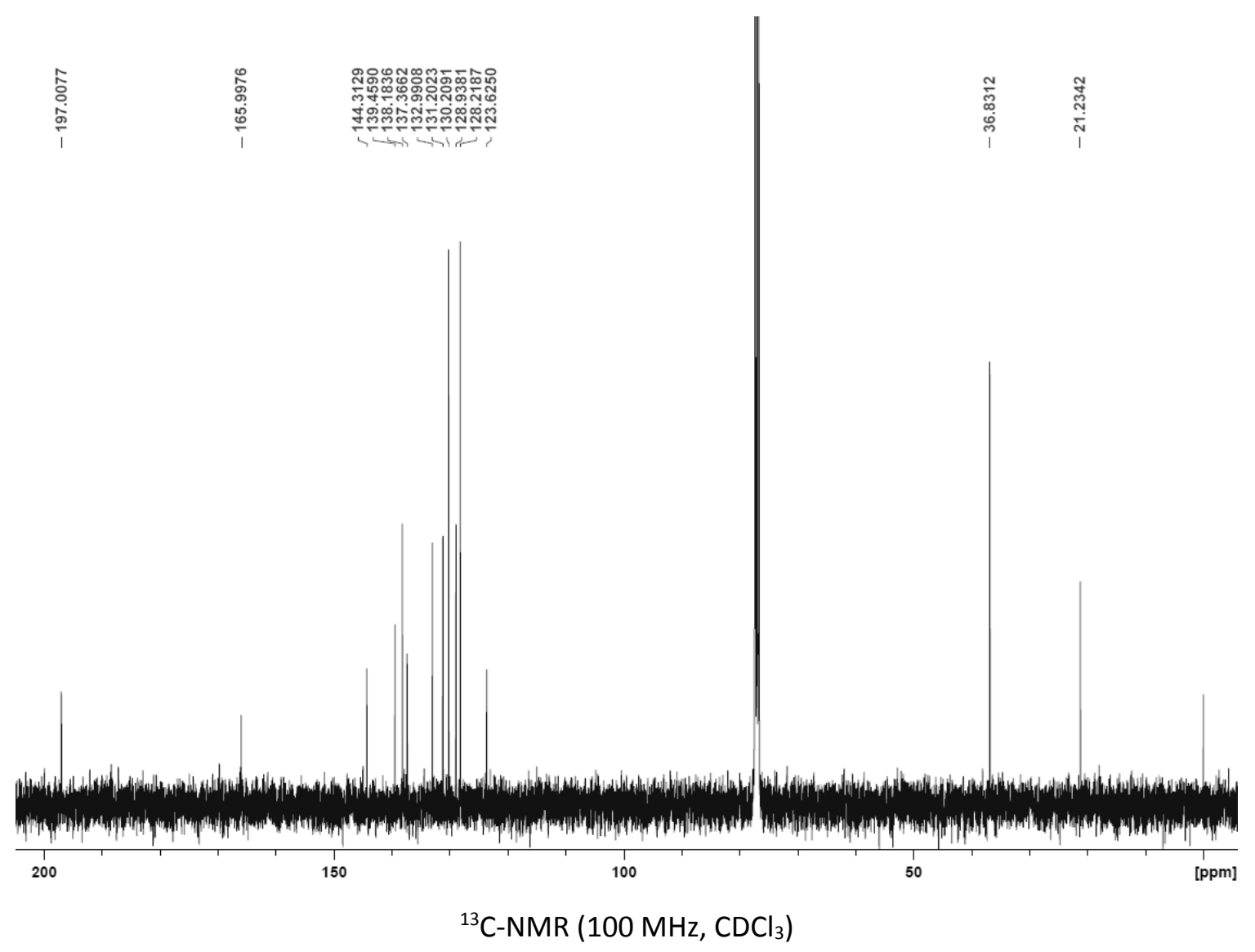




\subsubsection{S-(5-Methyl-2-pivaloylphenyl) Dimethylcarbamothioate (7)}

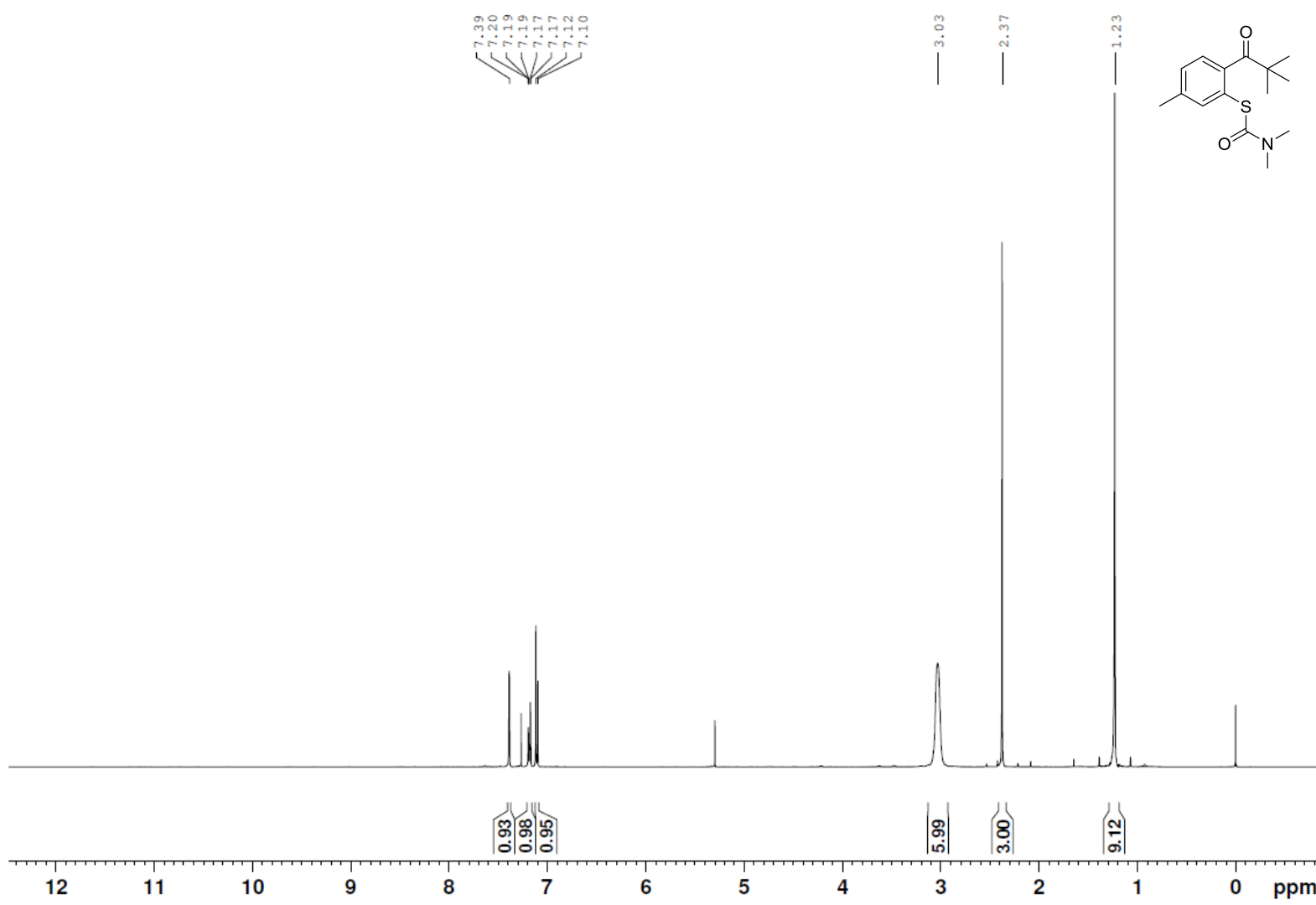

${ }^{1} \mathrm{H}-\mathrm{NMR}\left(400 \mathrm{MHz}, \mathrm{CDCl}_{3}\right)$

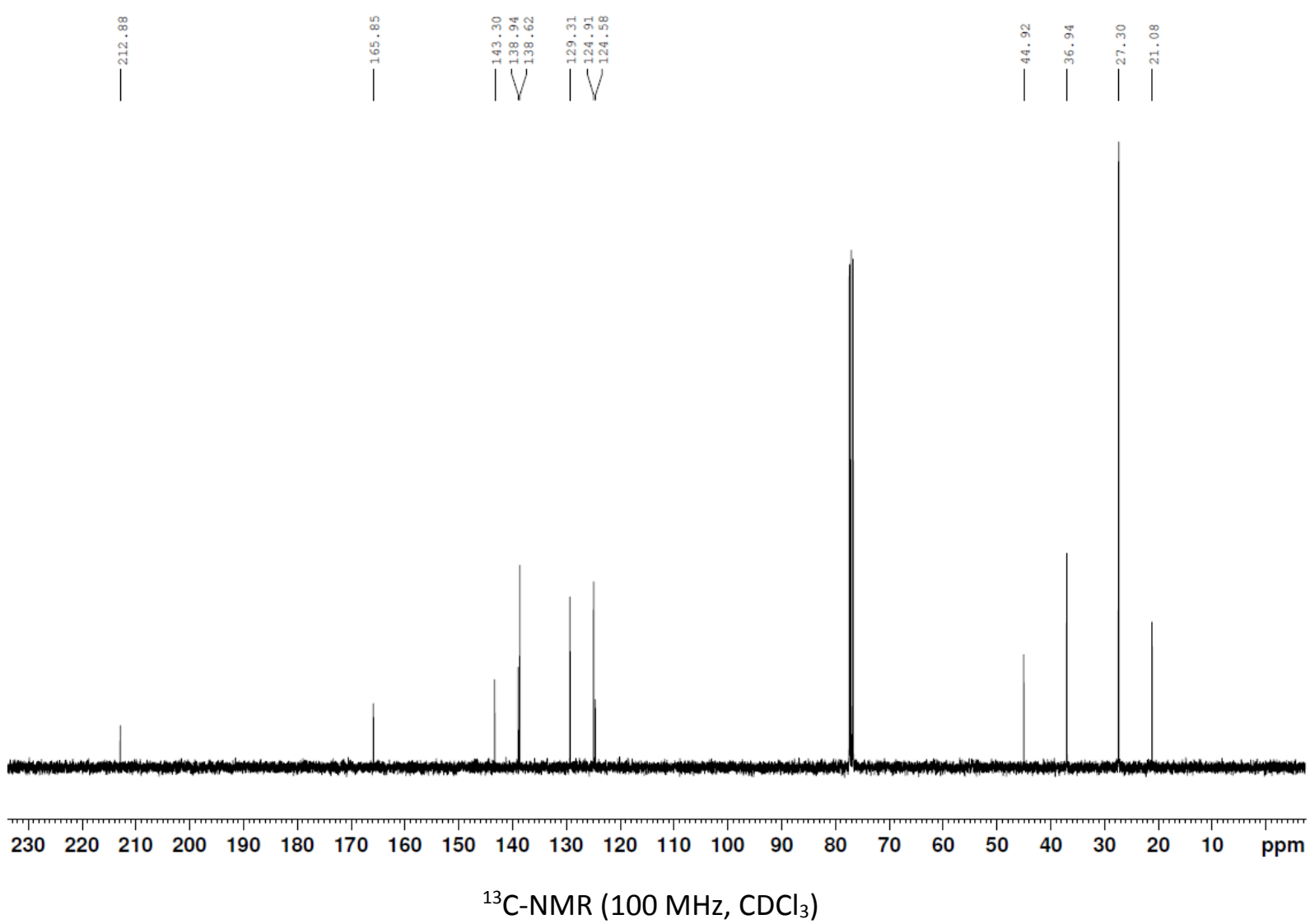


2.9.5 S-(2-Pivaloyl-5-(trifluoromethyl)phenyl) Dimethylcarbamothioate (8)
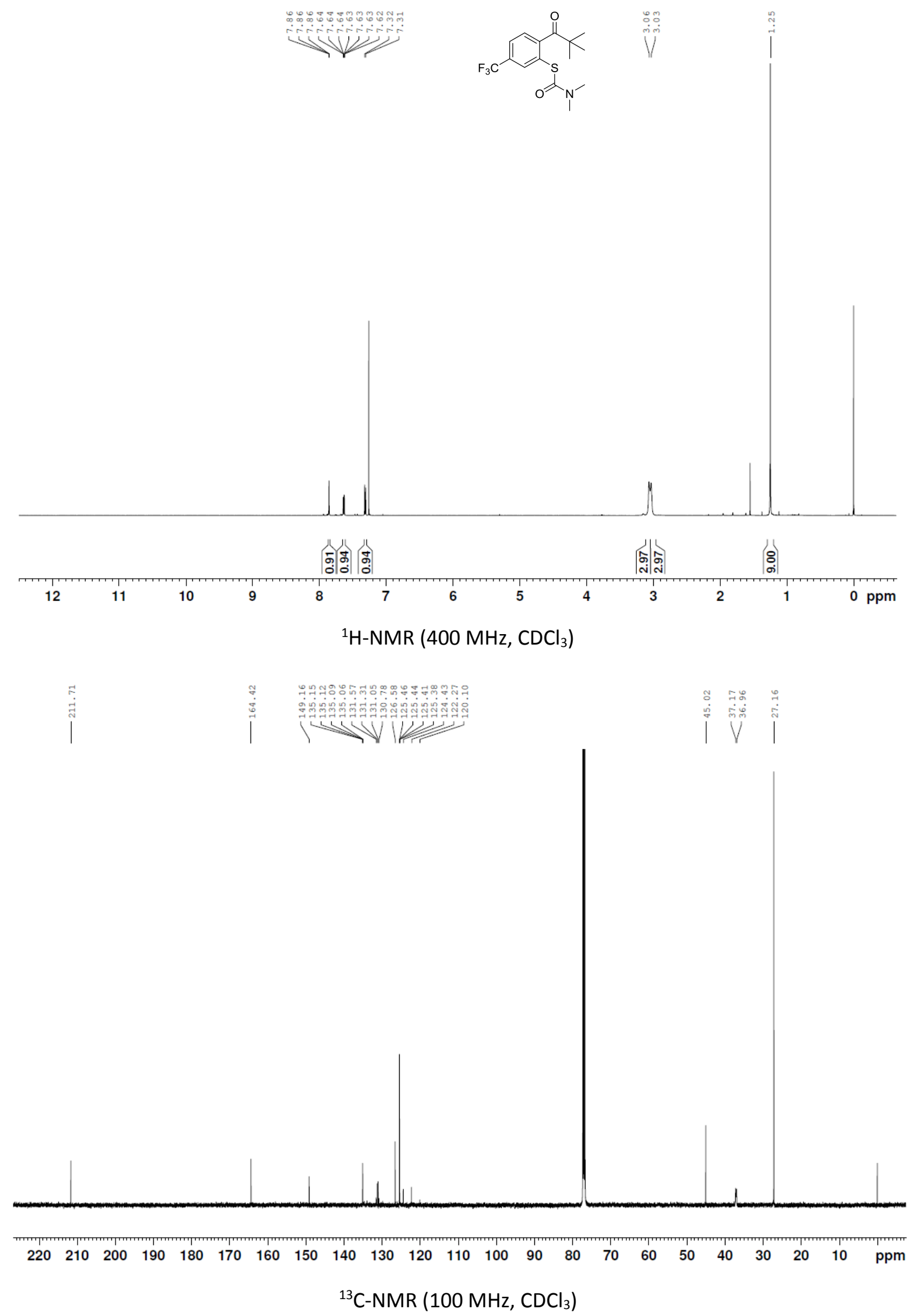


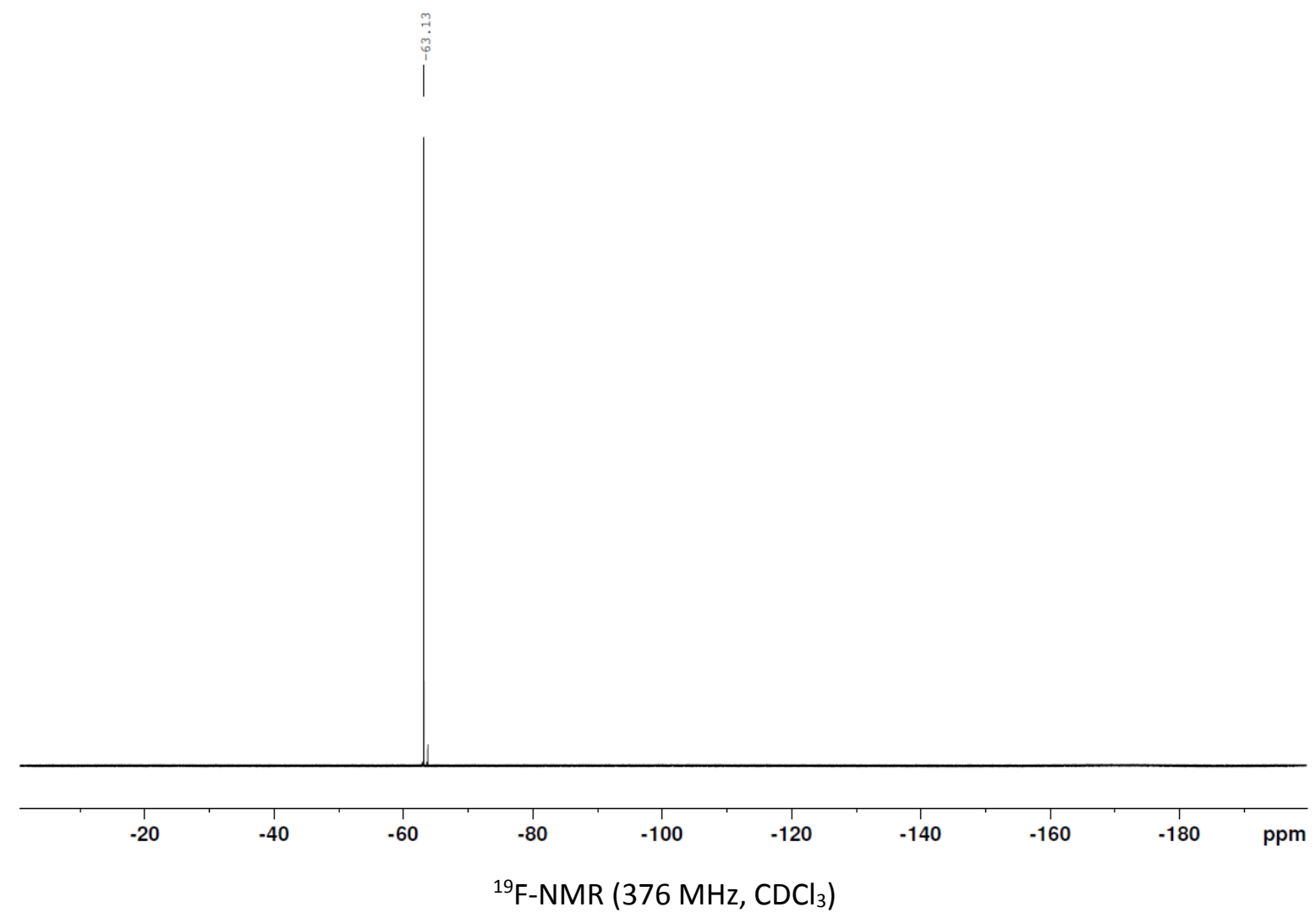




\subsection{NMR-Spectra of Benzothietes}

2.10.1 8-Phenyl-7-thiabicyclo[4.2.0]octa-1,3,5-triene (2)

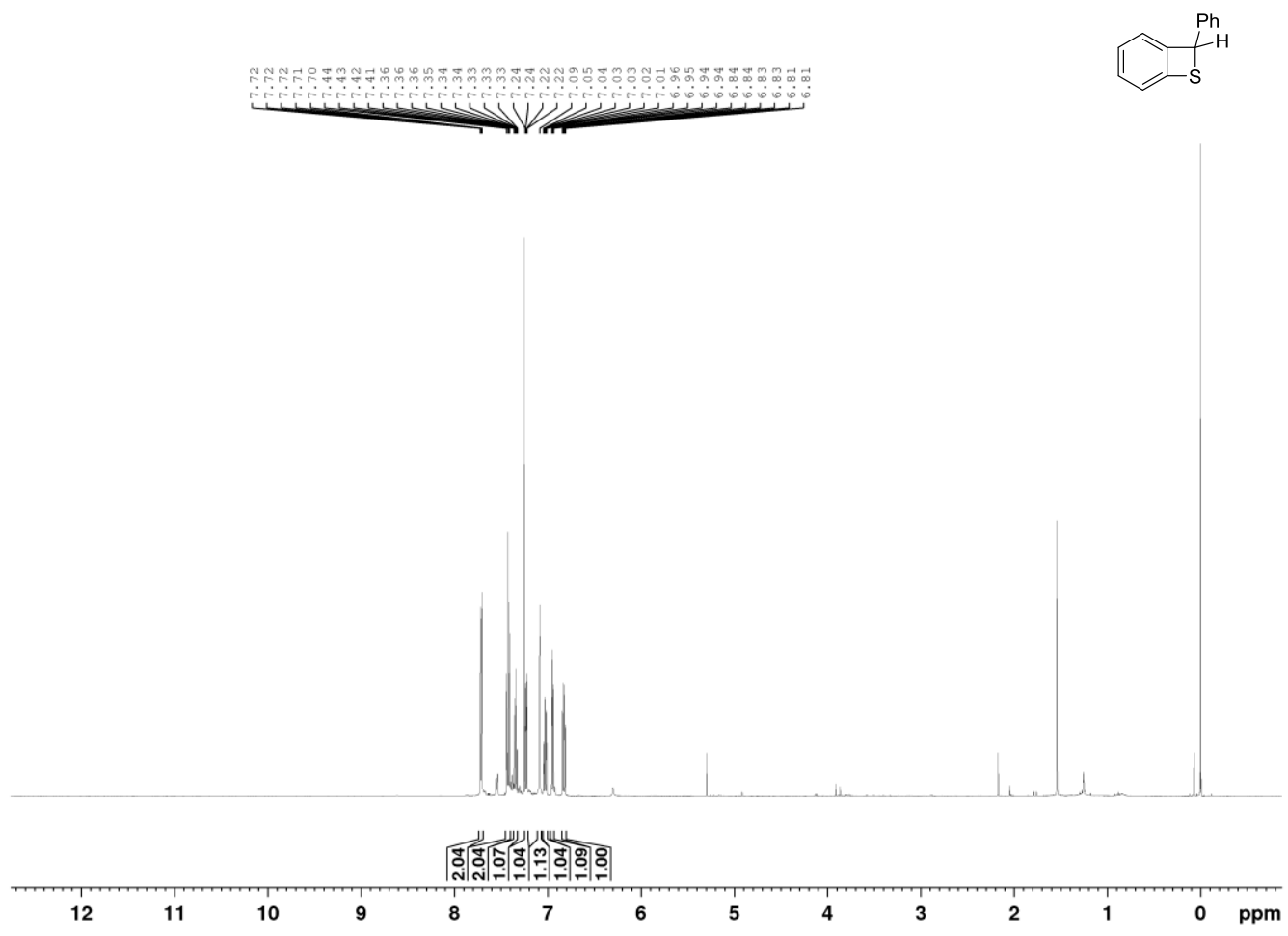

${ }^{1} \mathrm{H}-\mathrm{NMR}\left(500 \mathrm{MHz}, \mathrm{CDCl}_{3}\right)$

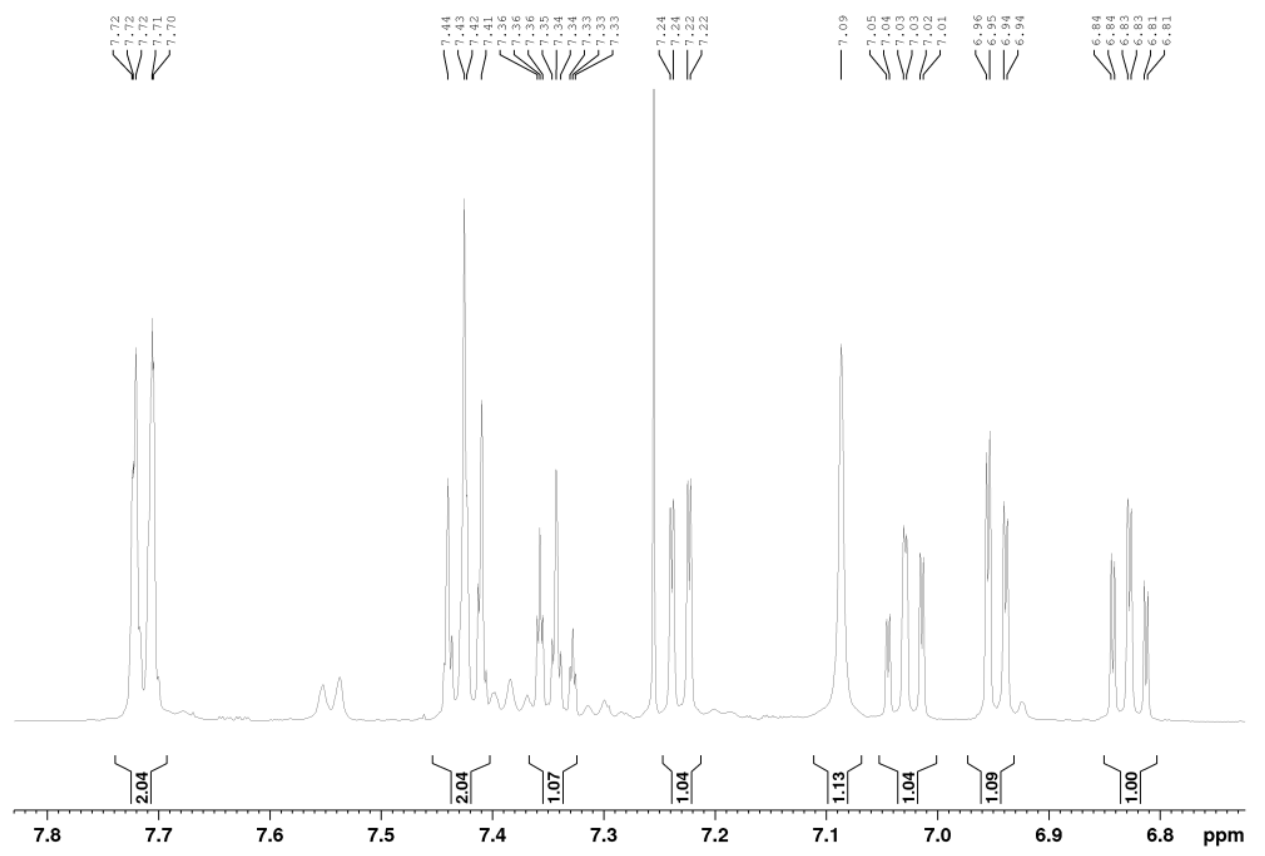

${ }^{1} \mathrm{H}-\mathrm{NMR}$ Detail $\left(500 \mathrm{MHz}, \mathrm{CDCl}_{3}\right)$ 


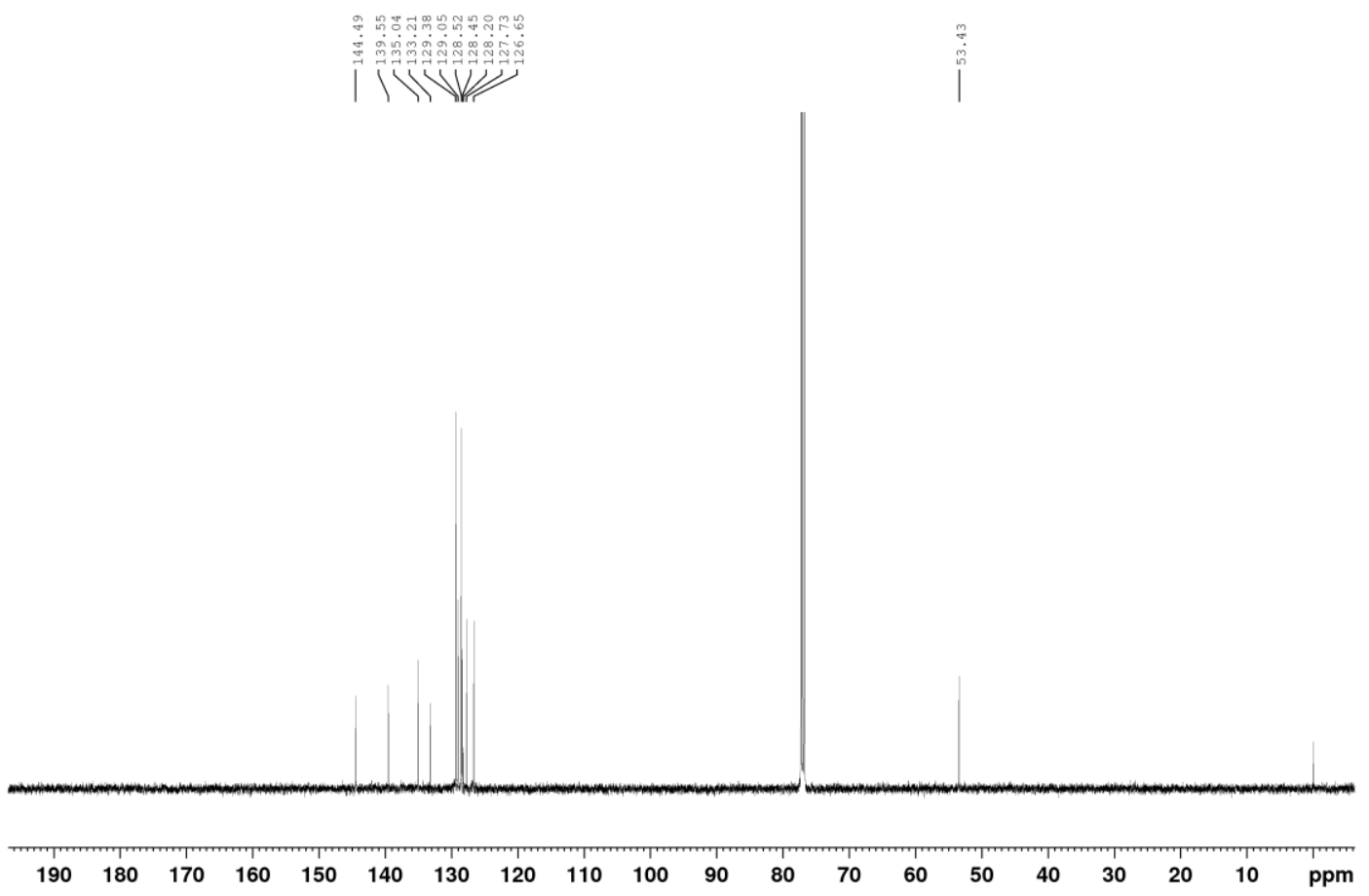

${ }^{13} \mathrm{C}-\mathrm{NMR}\left(125 \mathrm{MHz}, \mathrm{CDCl}_{3}\right)$

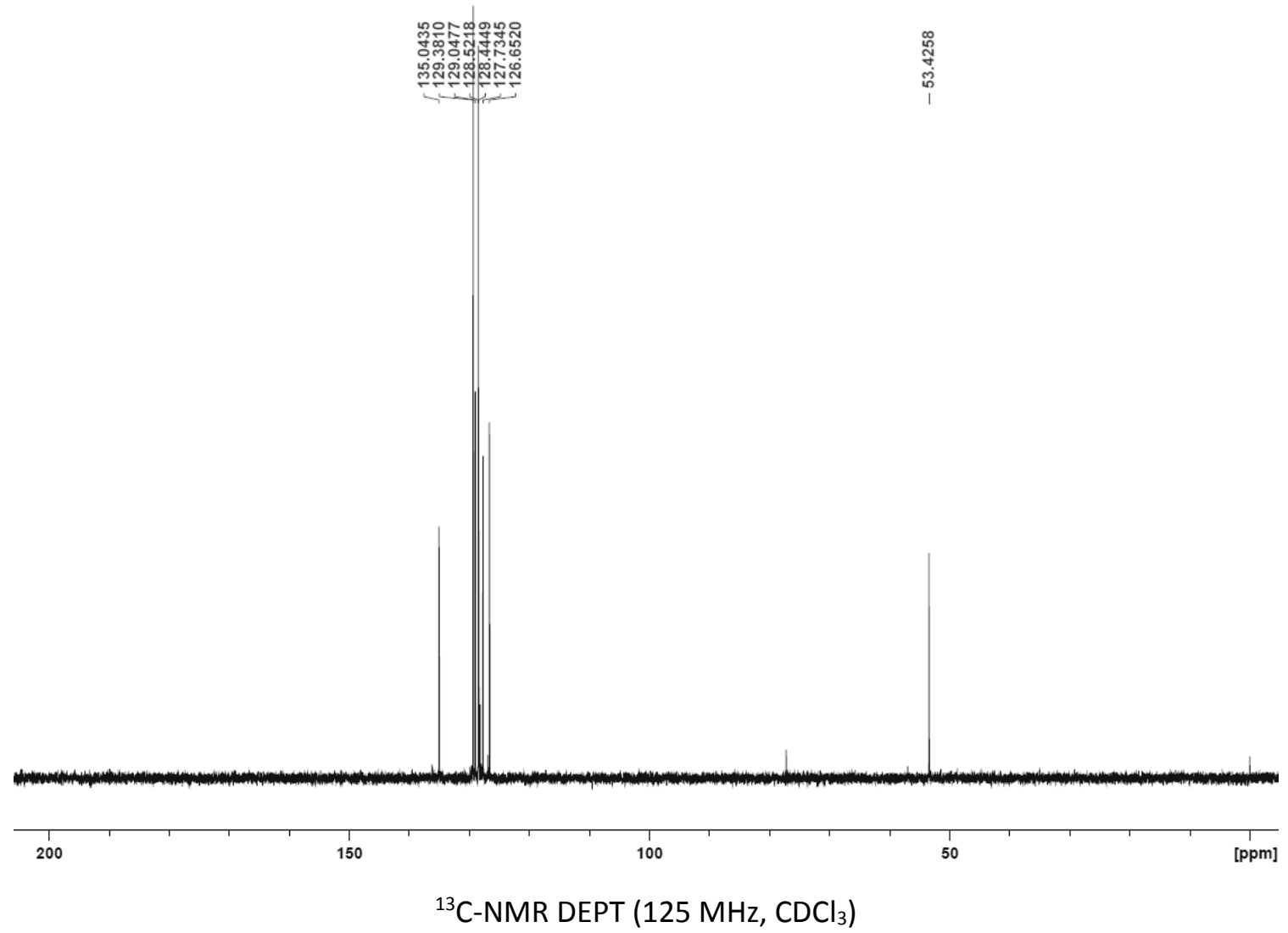



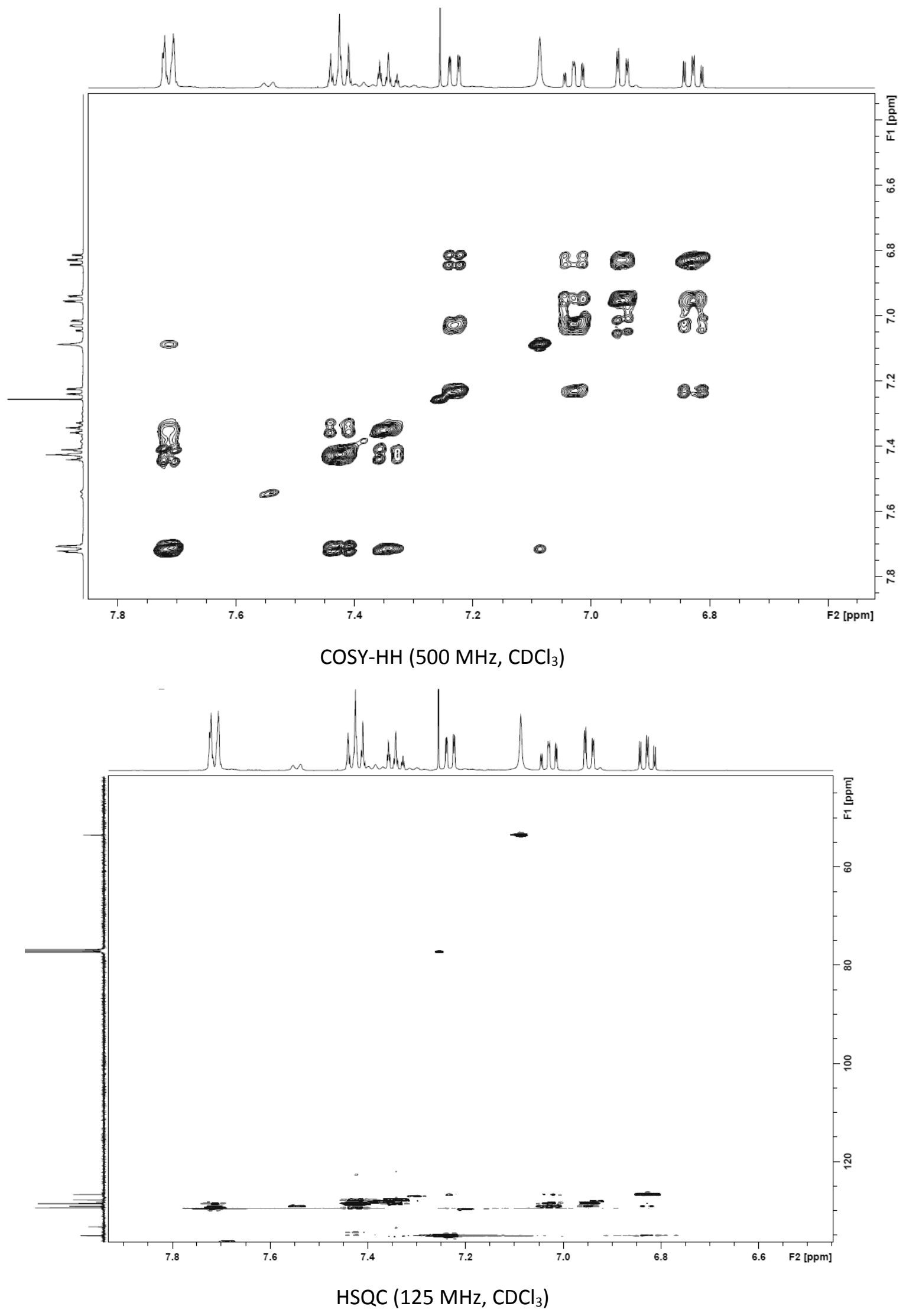


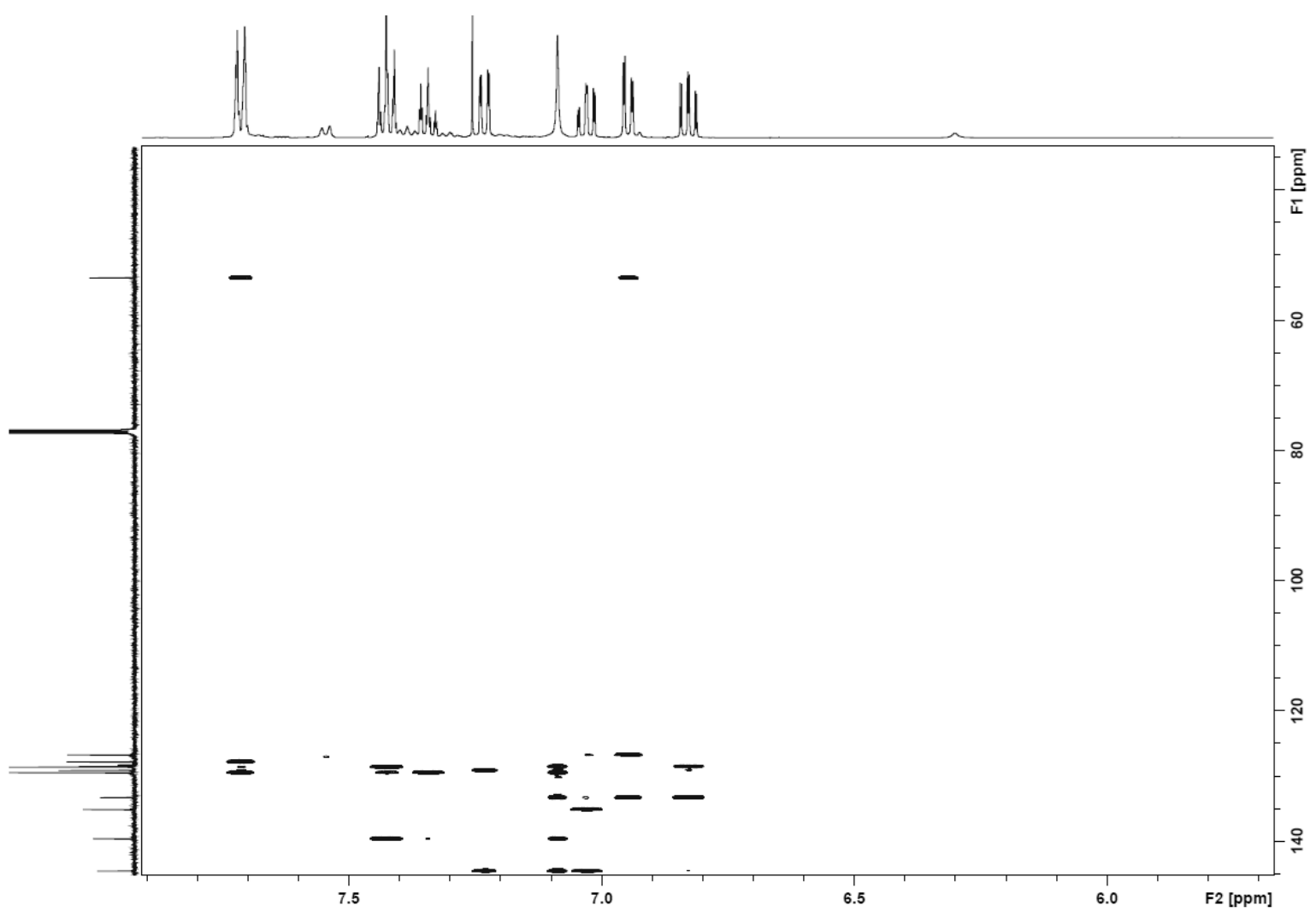

HMBC (500 MHz, $125 \mathrm{MHz}, \mathrm{CDCl}_{3}$ ) 


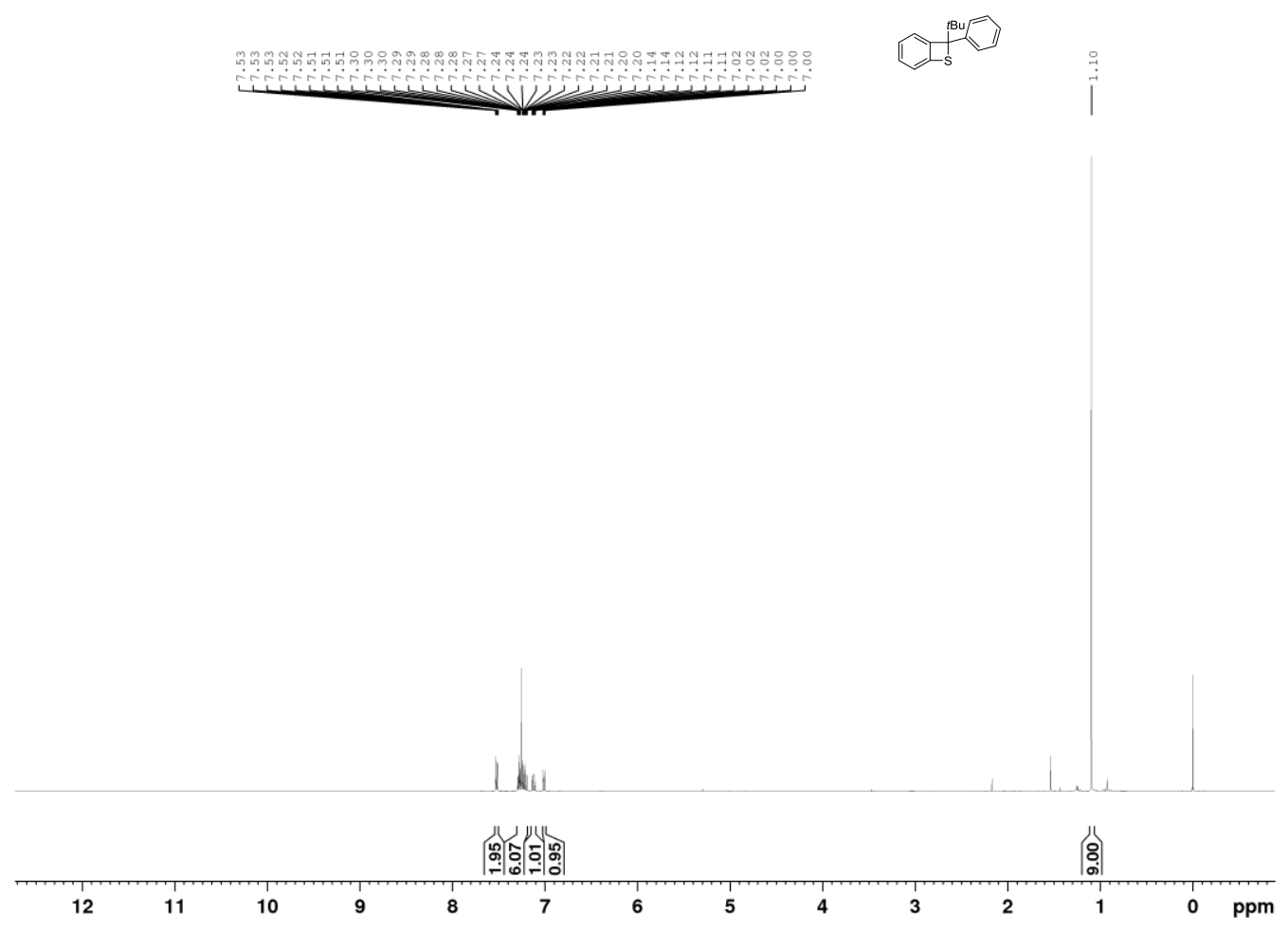

${ }^{1} \mathrm{H}-\mathrm{NMR}\left(500 \mathrm{MHz}, \mathrm{CDCl}_{3}\right)$

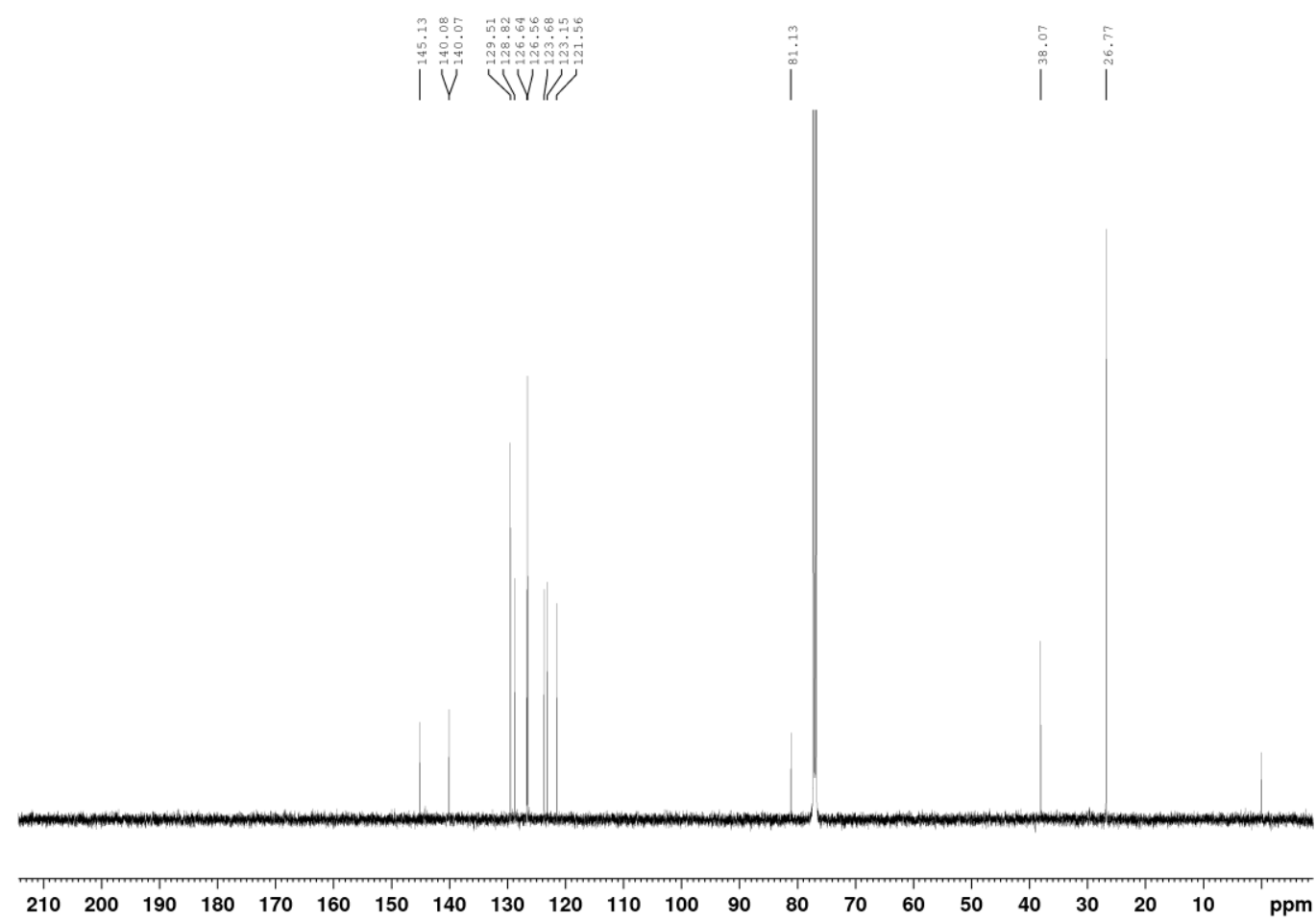

${ }^{13} \mathrm{C}-\mathrm{NMR}\left(125 \mathrm{MHz}, \mathrm{CDCl}_{3}\right)$ 


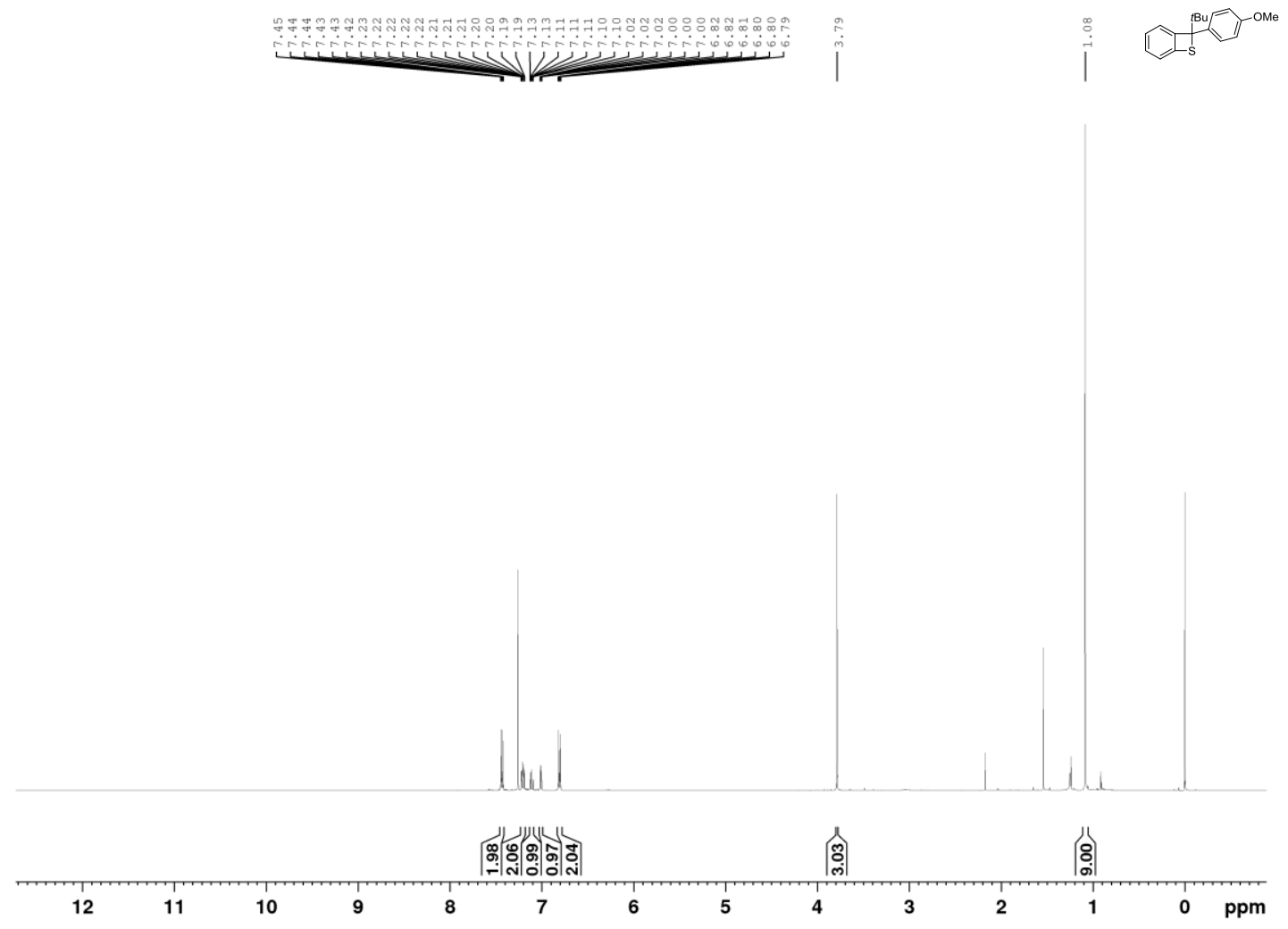

${ }^{1} \mathrm{H}-\mathrm{NMR}\left(500 \mathrm{MHz}, \mathrm{CDCl}_{3}\right)$

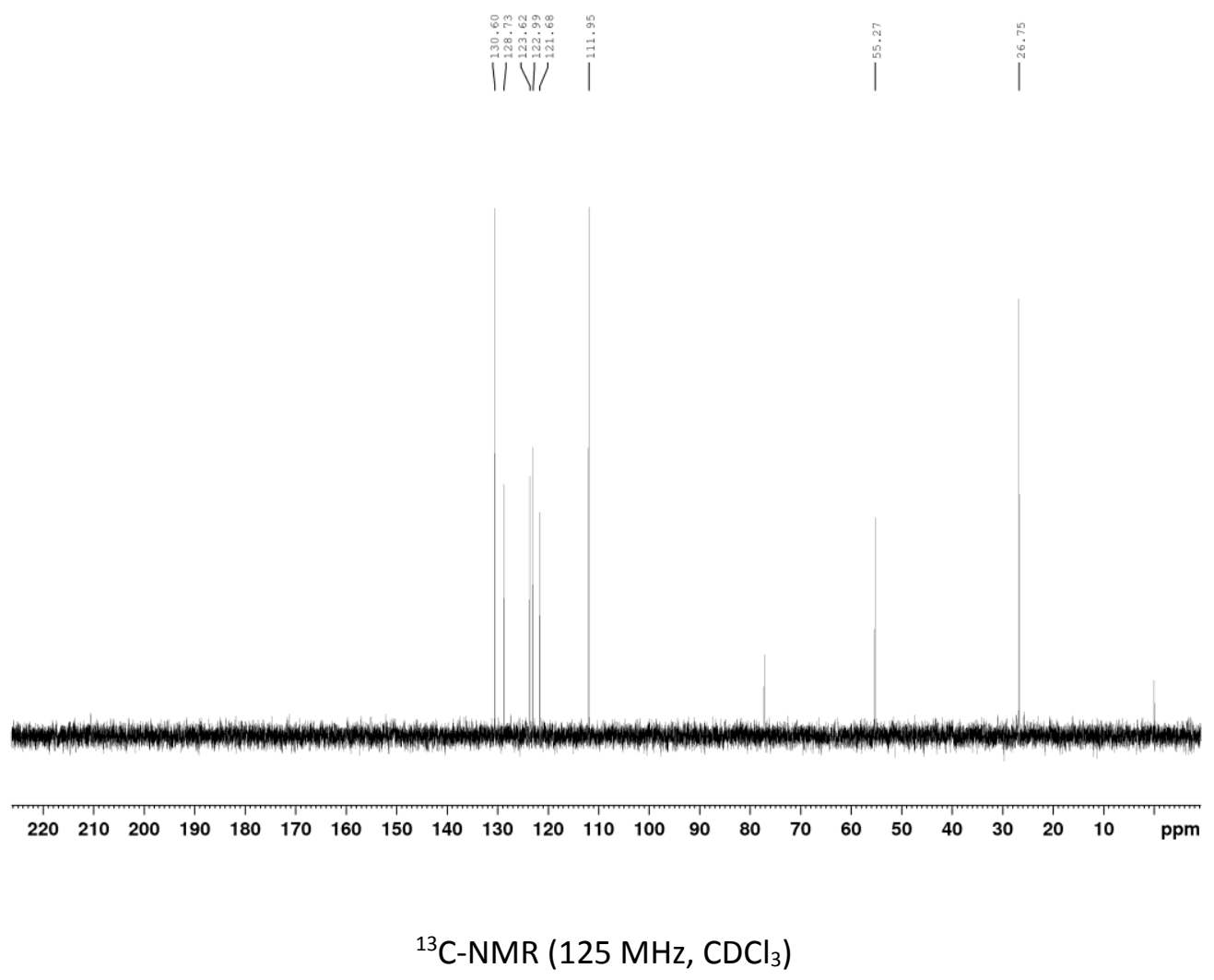



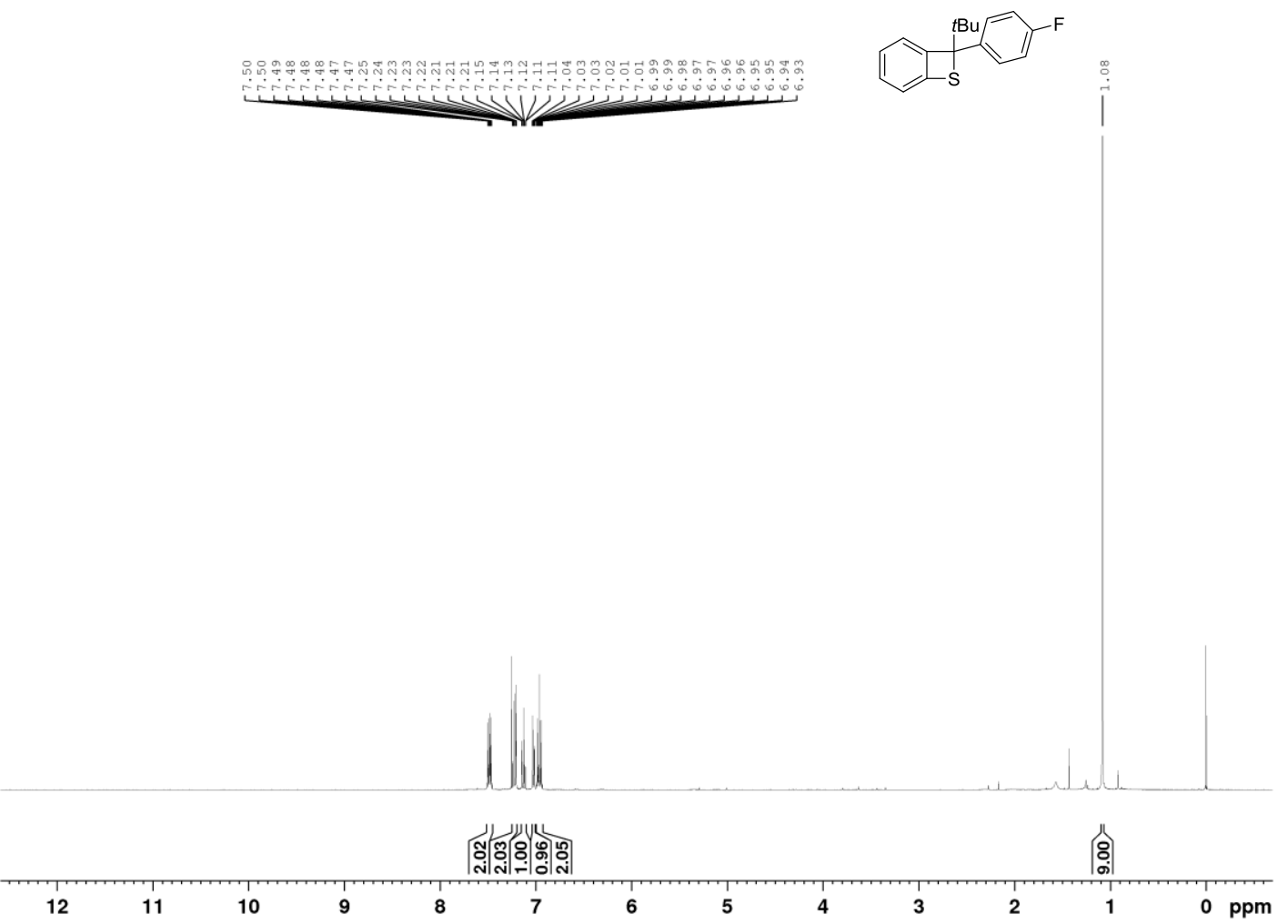

${ }^{1} \mathrm{H}-\mathrm{NMR}\left(400 \mathrm{MHz}, \mathrm{CDCl}_{3}\right)$

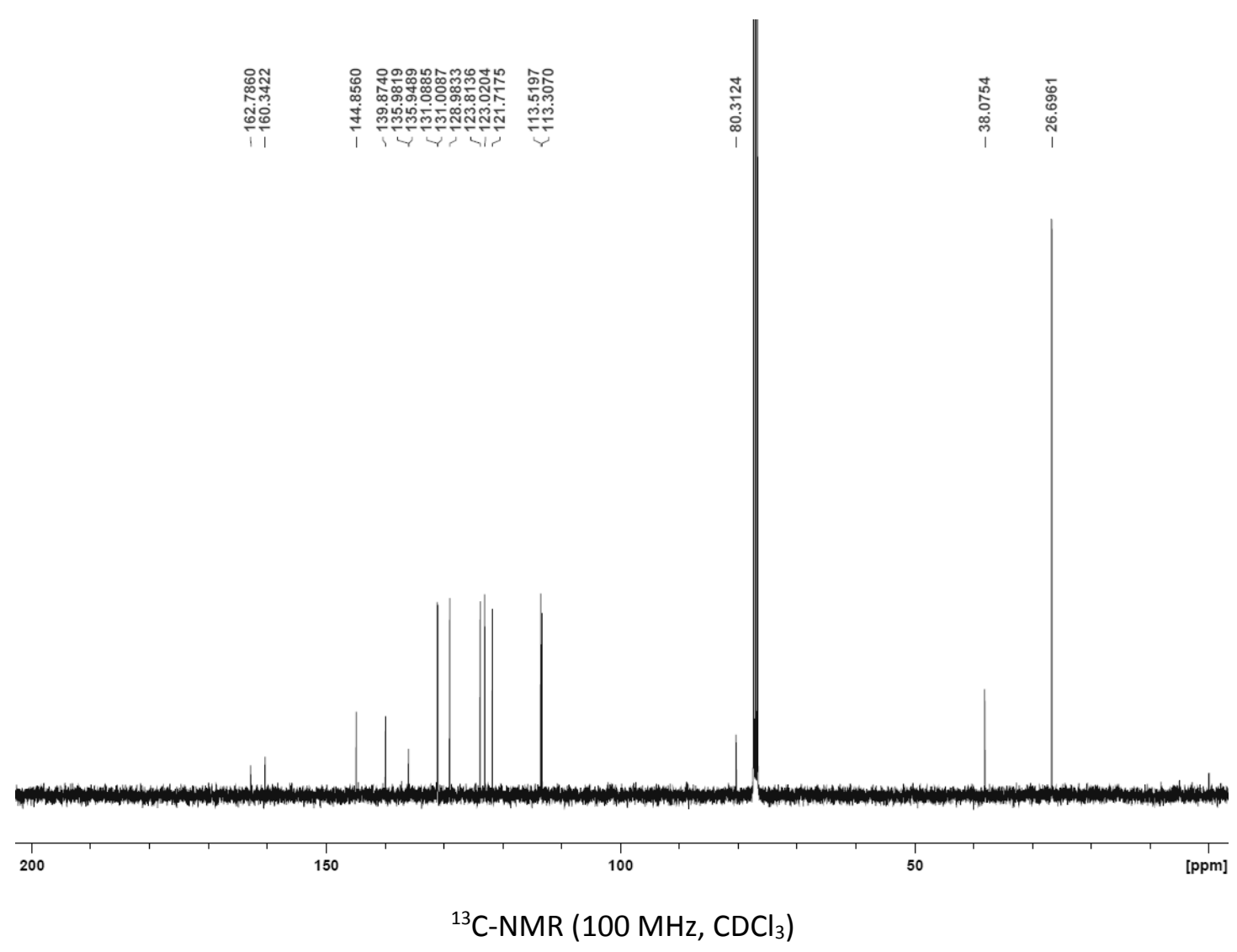




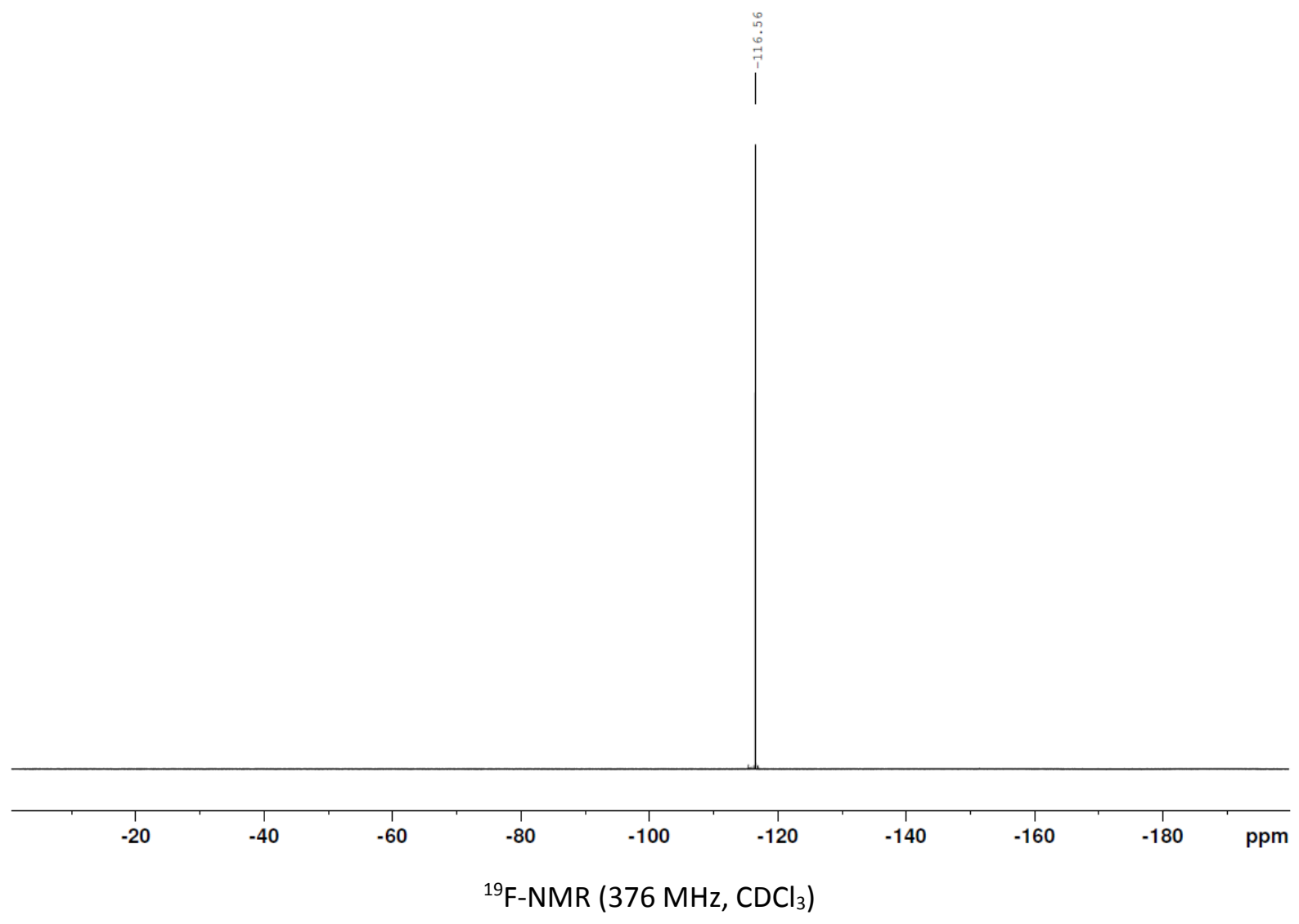




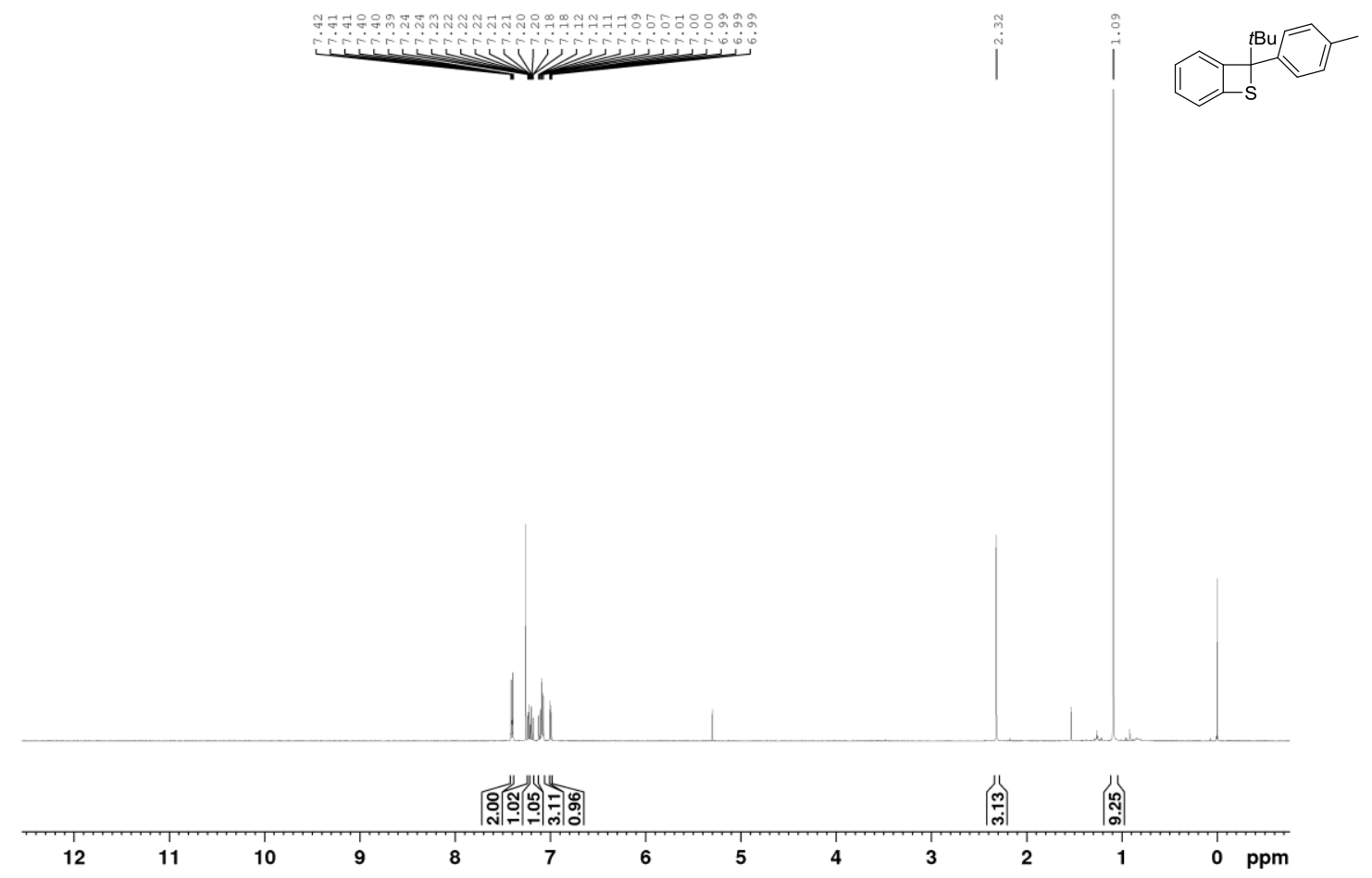

${ }^{1} \mathrm{H}-\mathrm{NMR}\left(400 \mathrm{MHz}, \mathrm{CDCl}_{3}\right.$ )

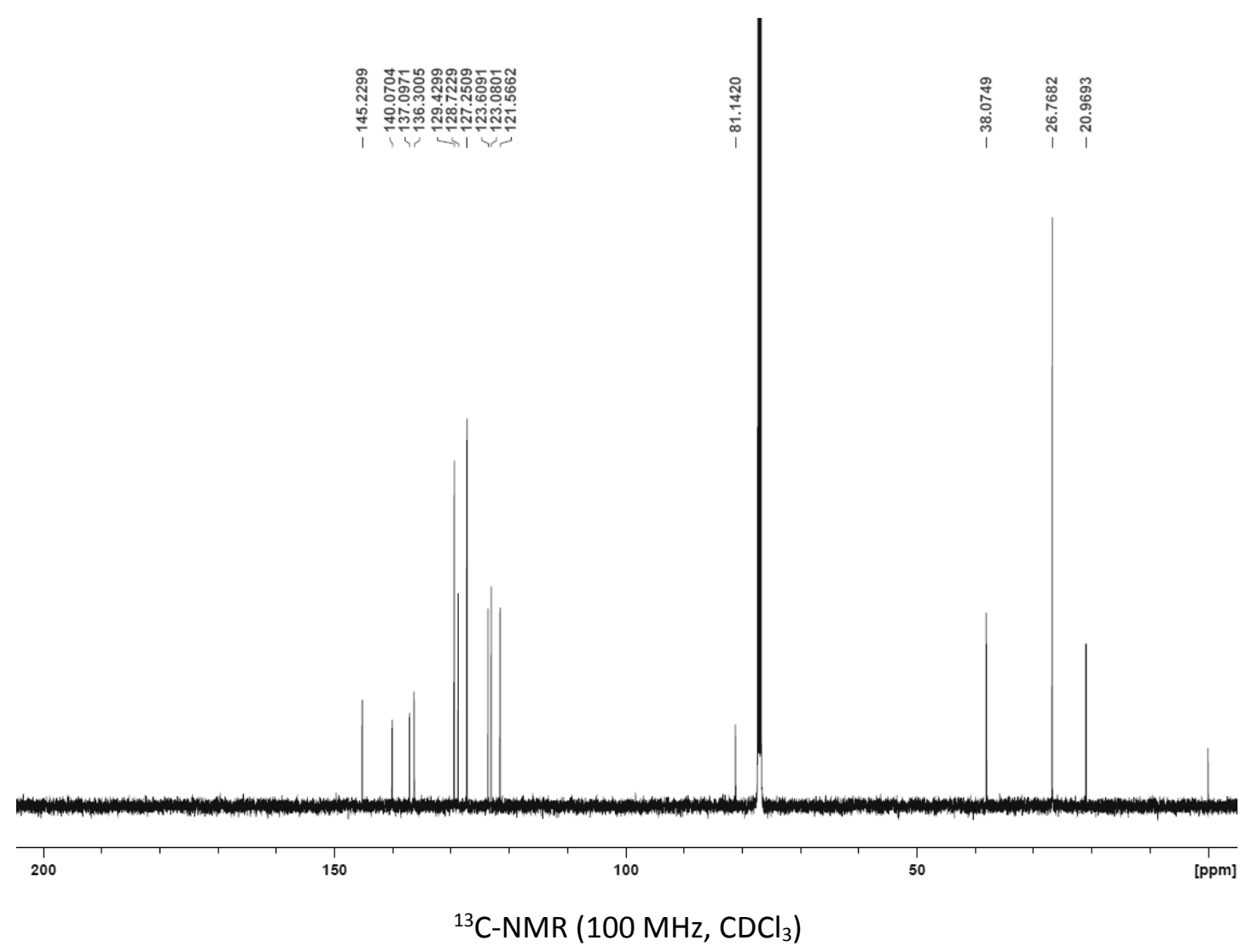


2.10.6 8-Benzyl-8-(tert-butyl)-7-thiabicyclo[4.2.0]octa-1,3,5-triene (9e)

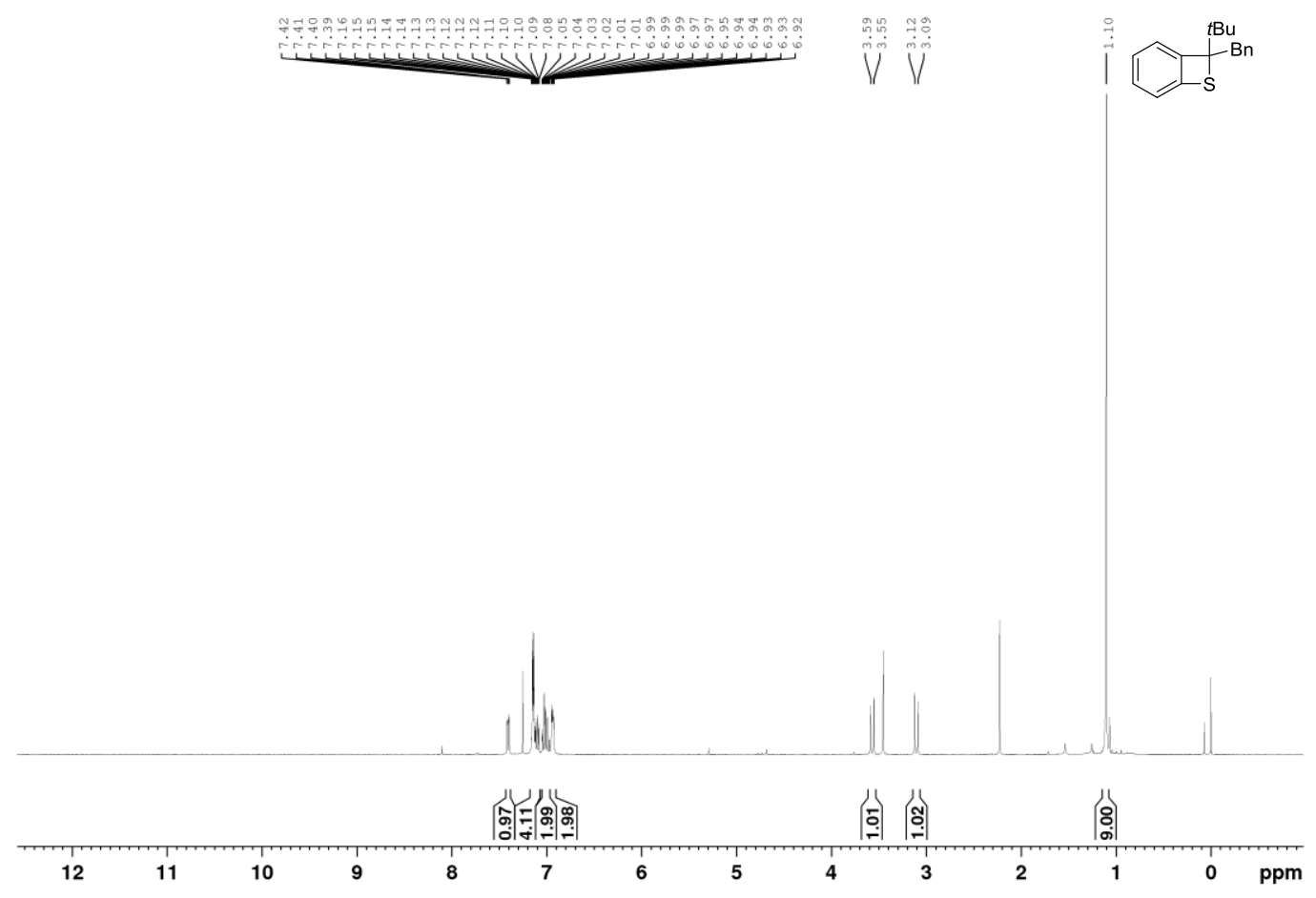

${ }^{1} \mathrm{H}-\mathrm{NMR}\left(400 \mathrm{MHz}, \mathrm{CDCl}_{3}\right)$

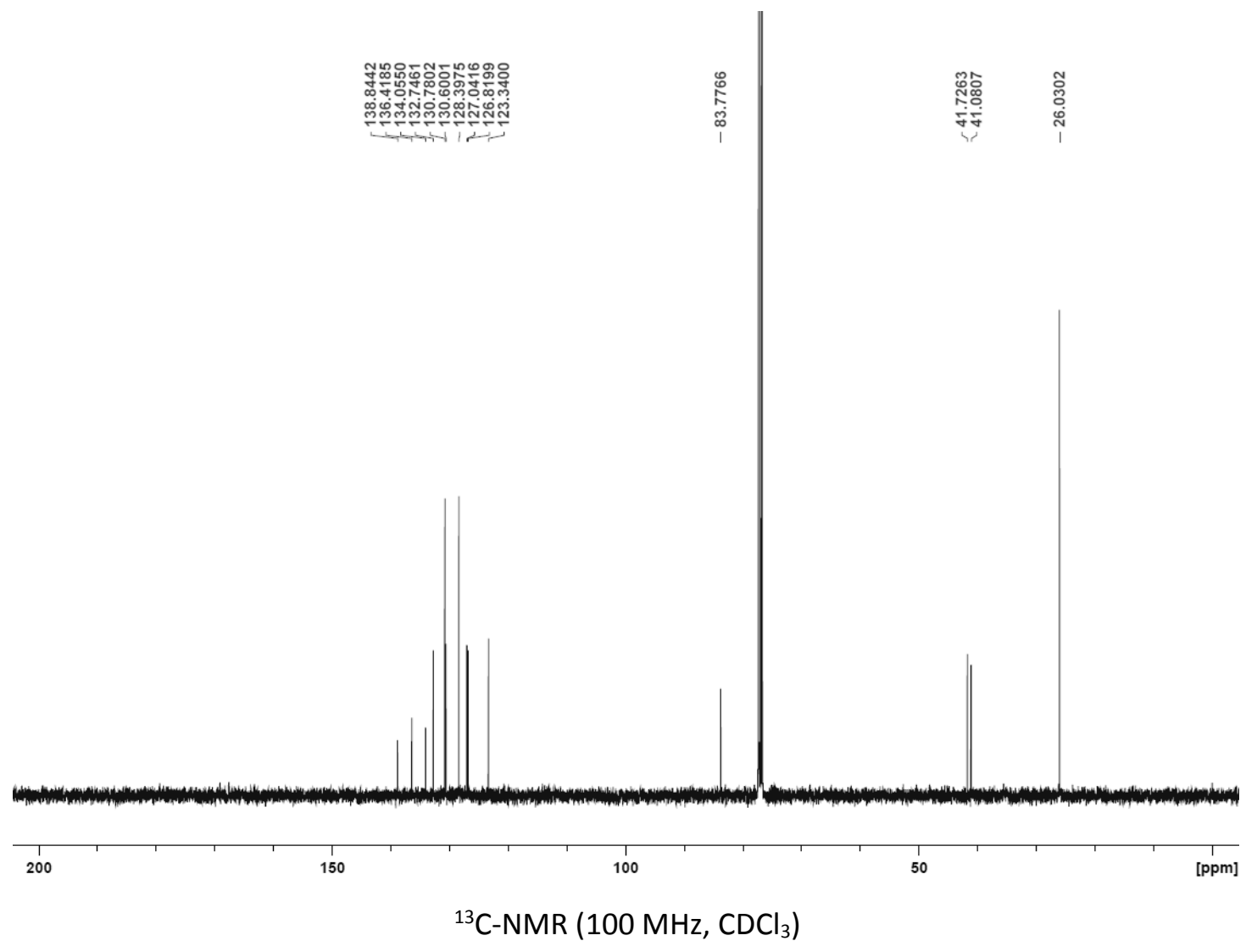


2.10.7 8-(tert-Butyl)-8-(4-chlorophenyl)-7-thiabicyclo[4.2.0]octa-1,3,5-triene (9f)

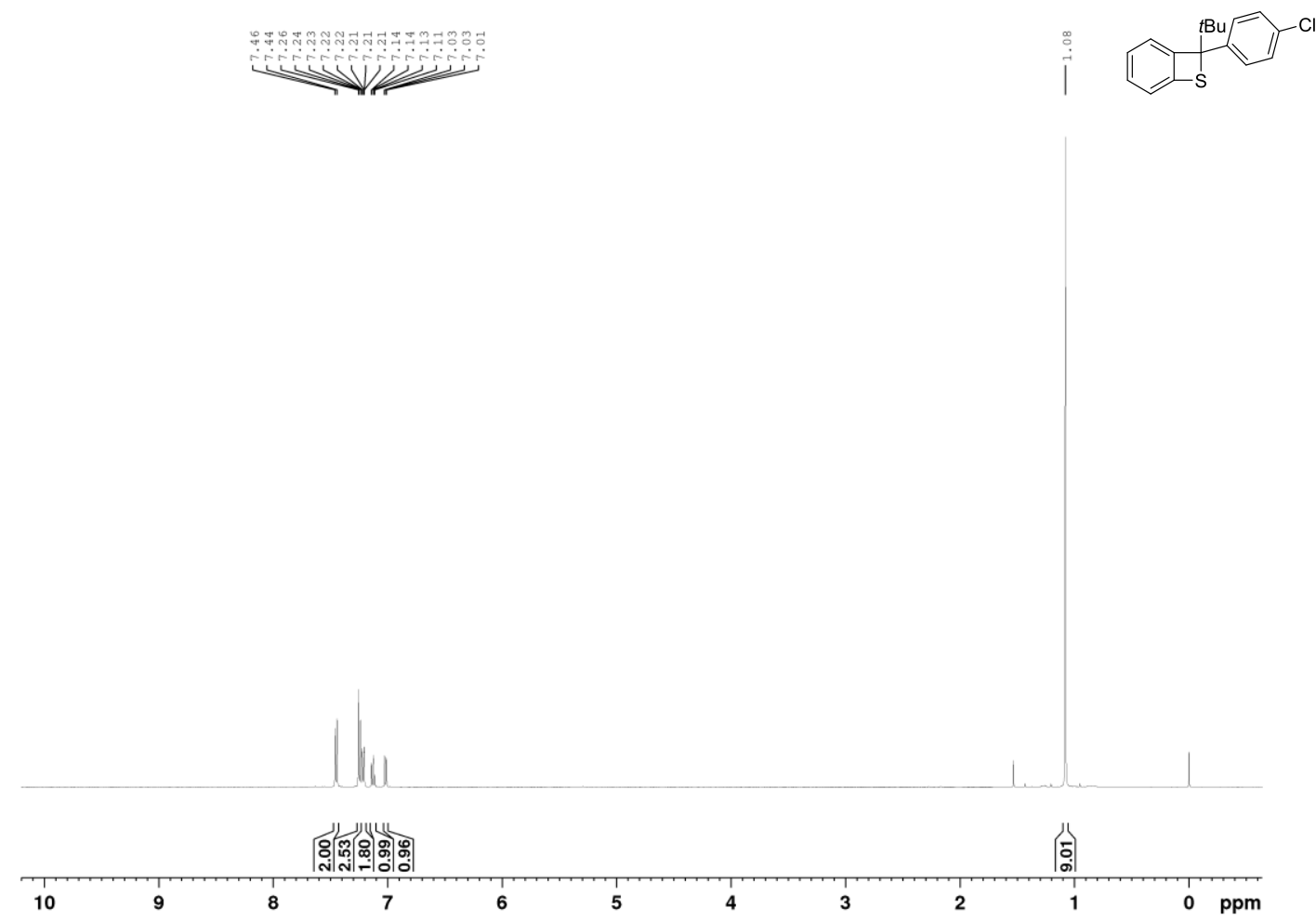

${ }^{1} \mathrm{H}-\mathrm{NMR}\left(500 \mathrm{MHz}, \mathrm{CDCl}_{3}\right)$

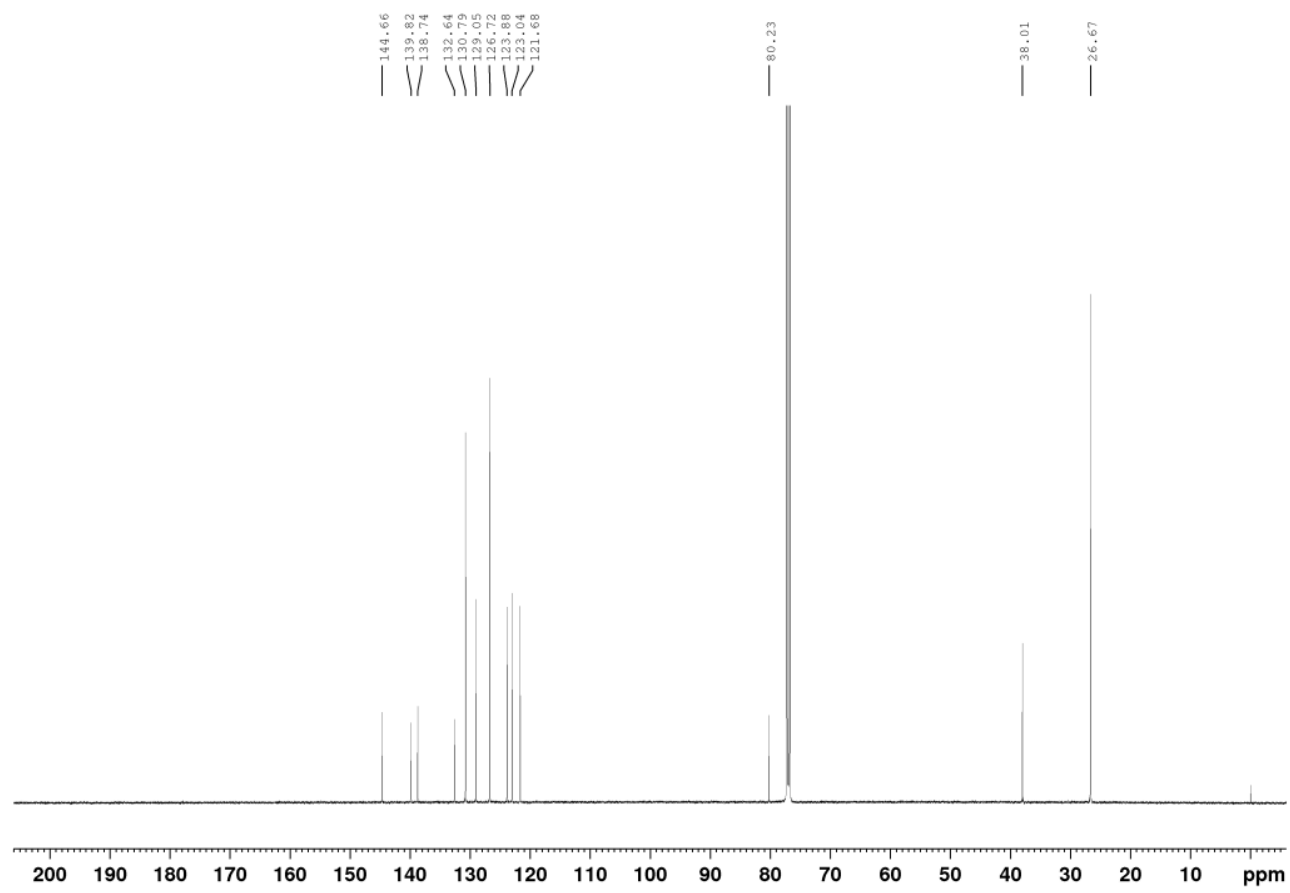

${ }^{13} \mathrm{C}-\mathrm{NMR}\left(125 \mathrm{MHz}, \mathrm{CDCl}_{3}\right)$ 


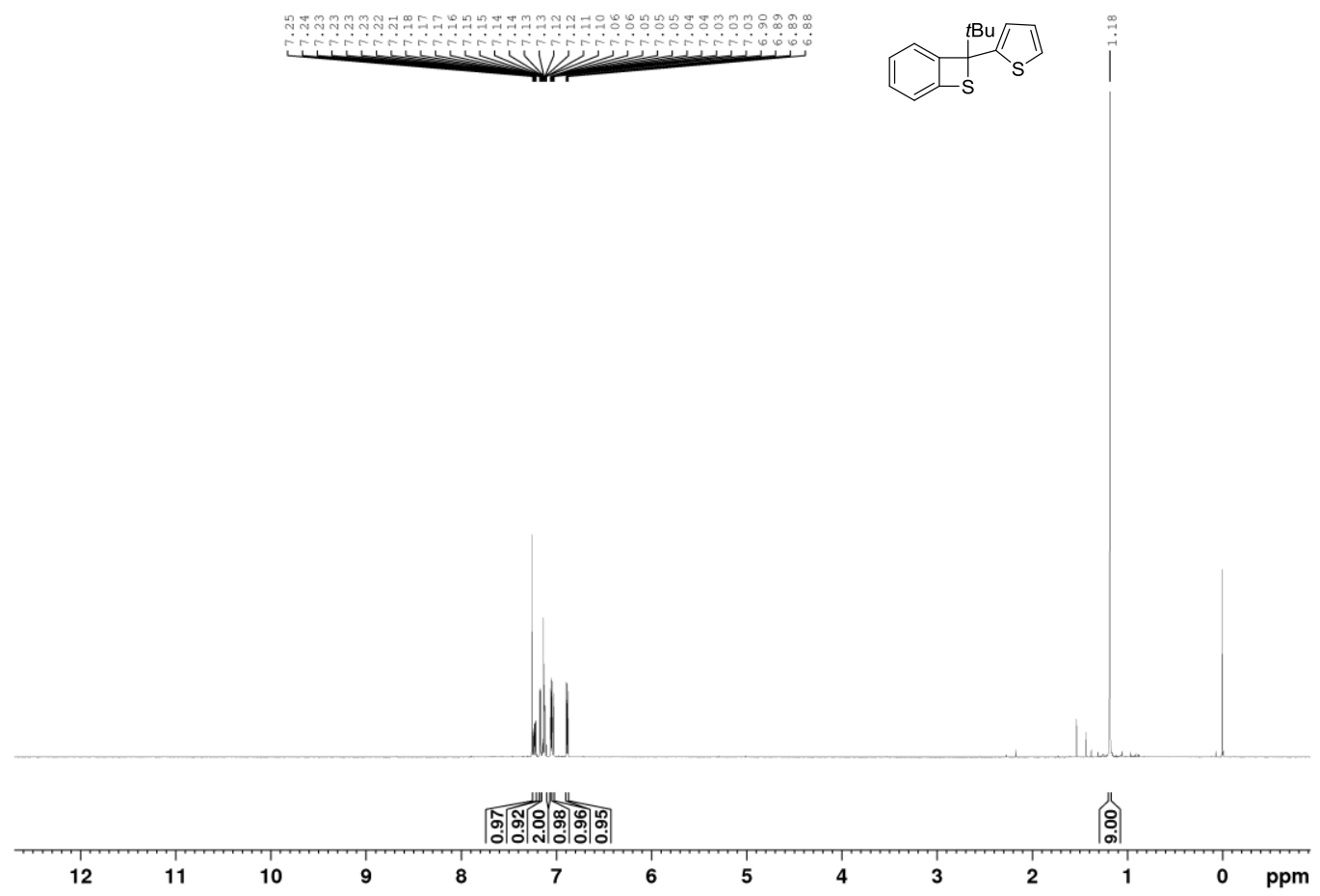

${ }^{1} \mathrm{H}-\mathrm{NMR}\left(500 \mathrm{MHz}, \mathrm{CDCl}_{3}\right)$

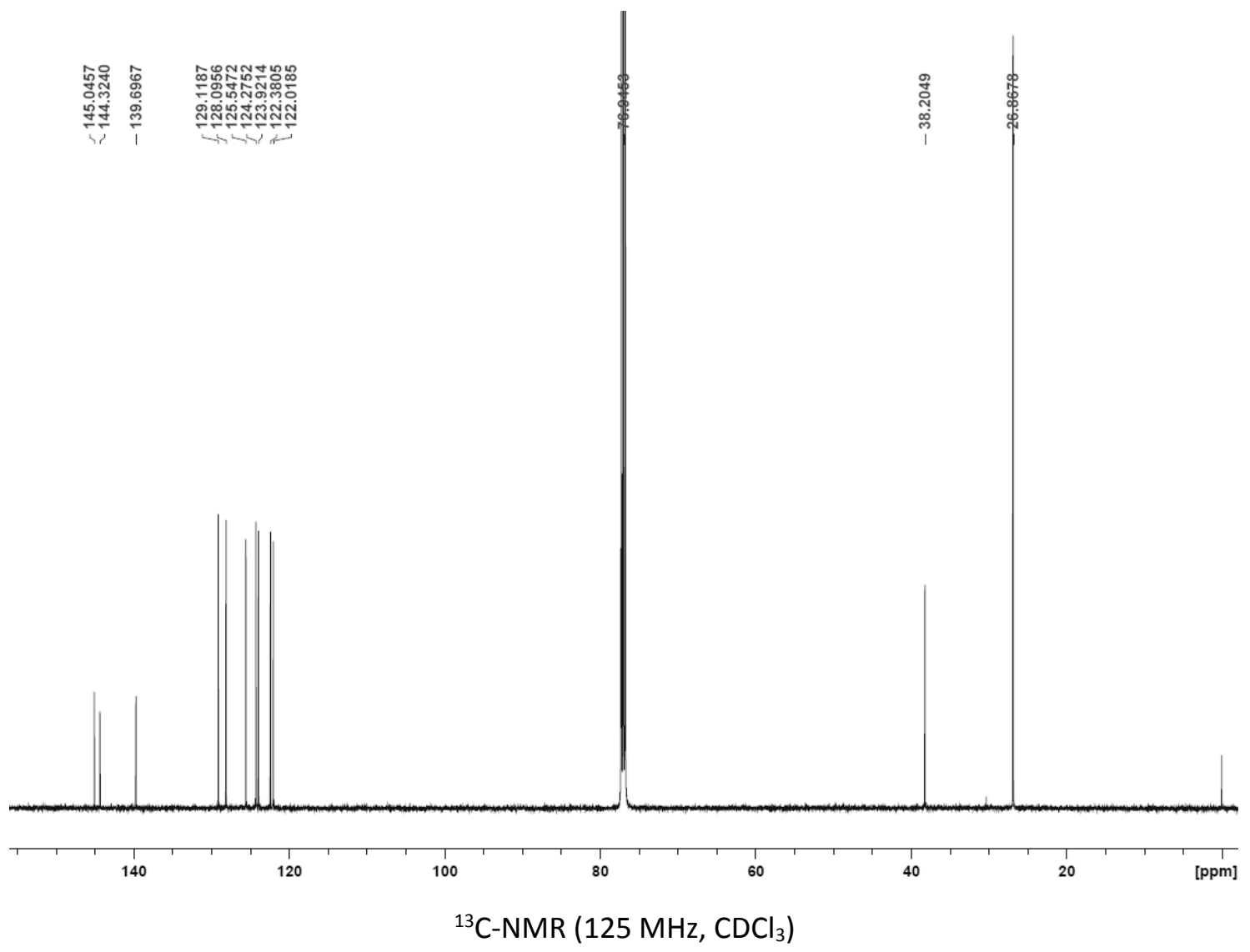




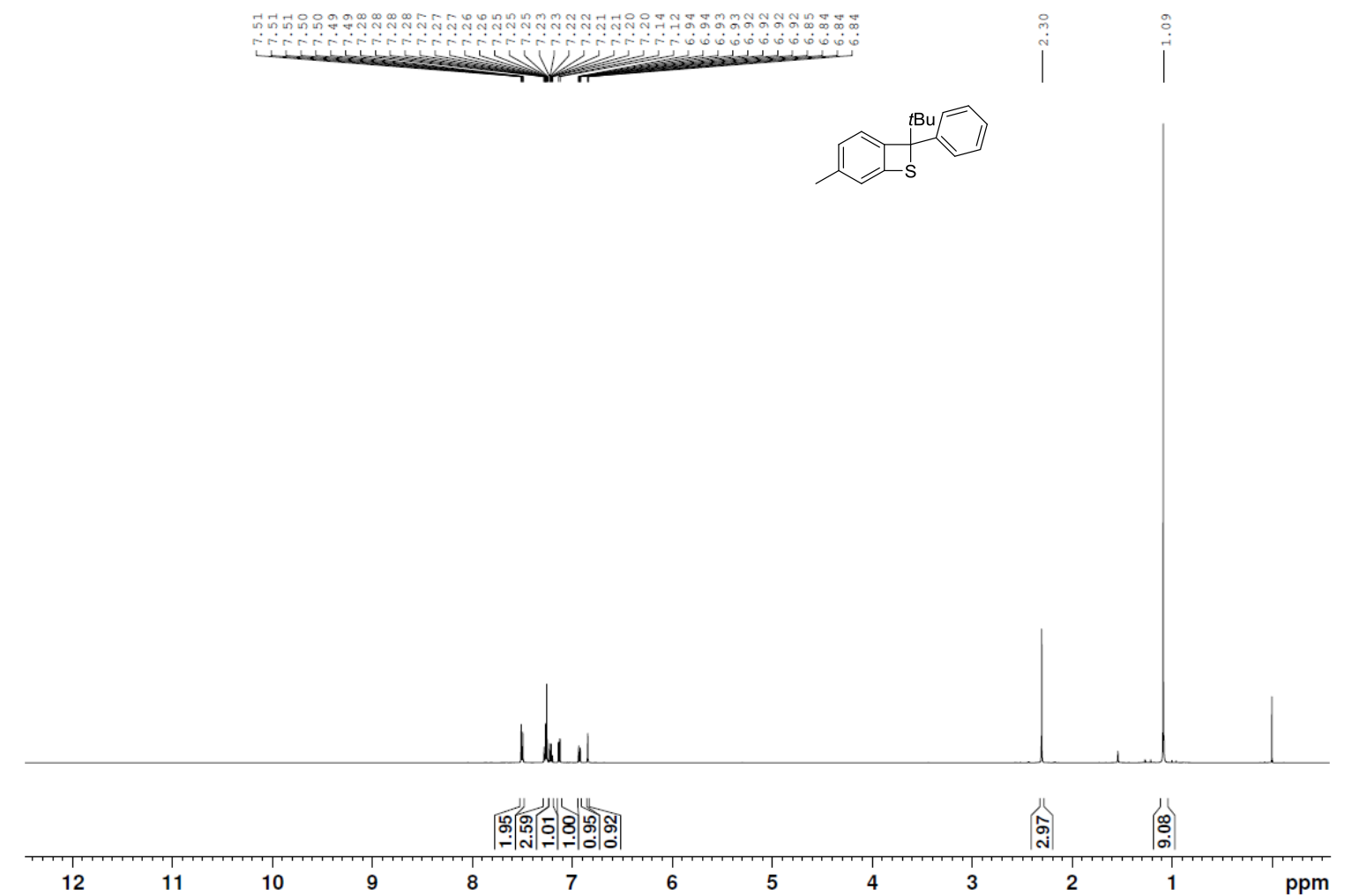

${ }^{1} \mathrm{H}-\mathrm{NMR}\left(500 \mathrm{MHz}, \mathrm{CDCl}_{3}\right)$

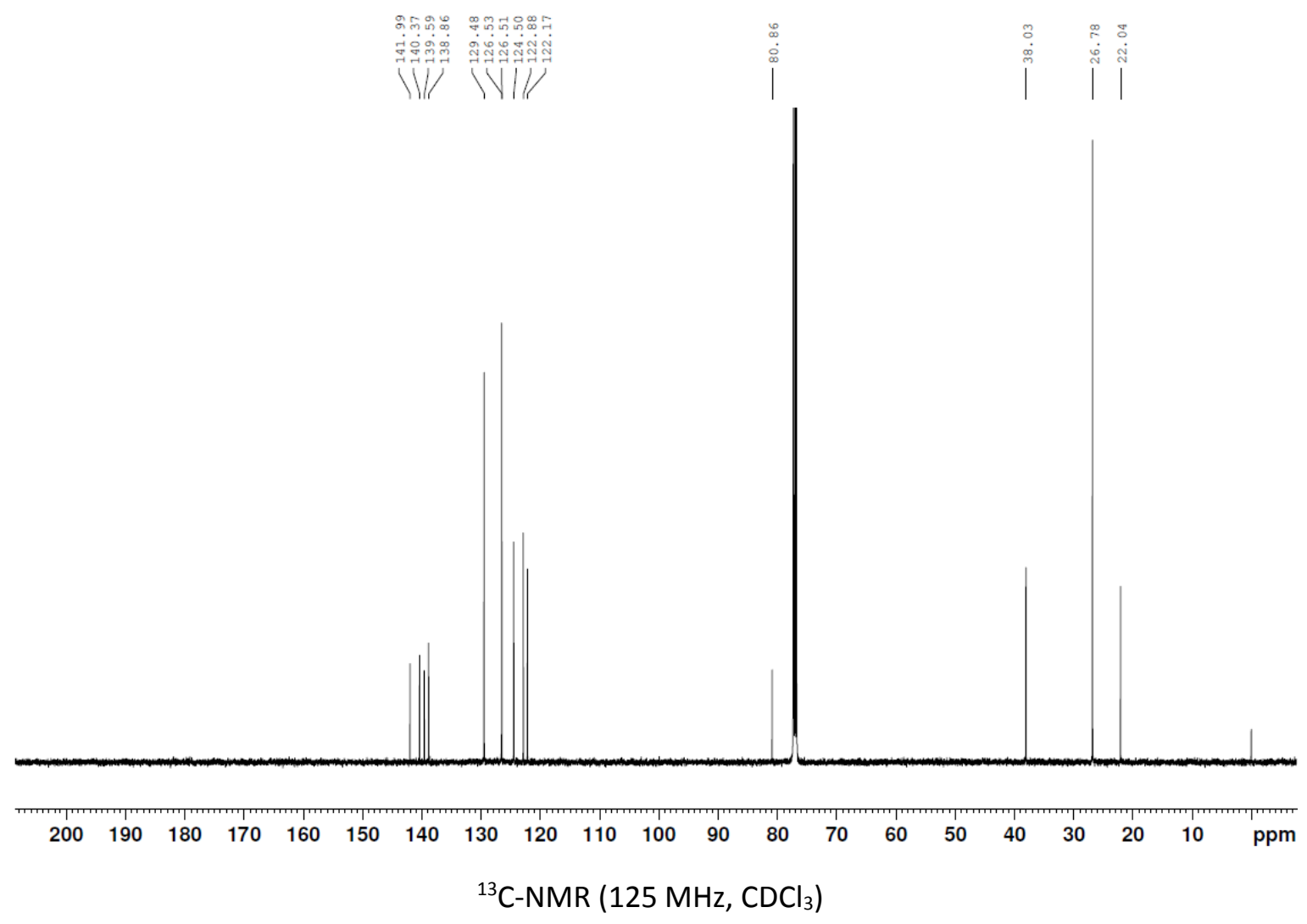


(10b)

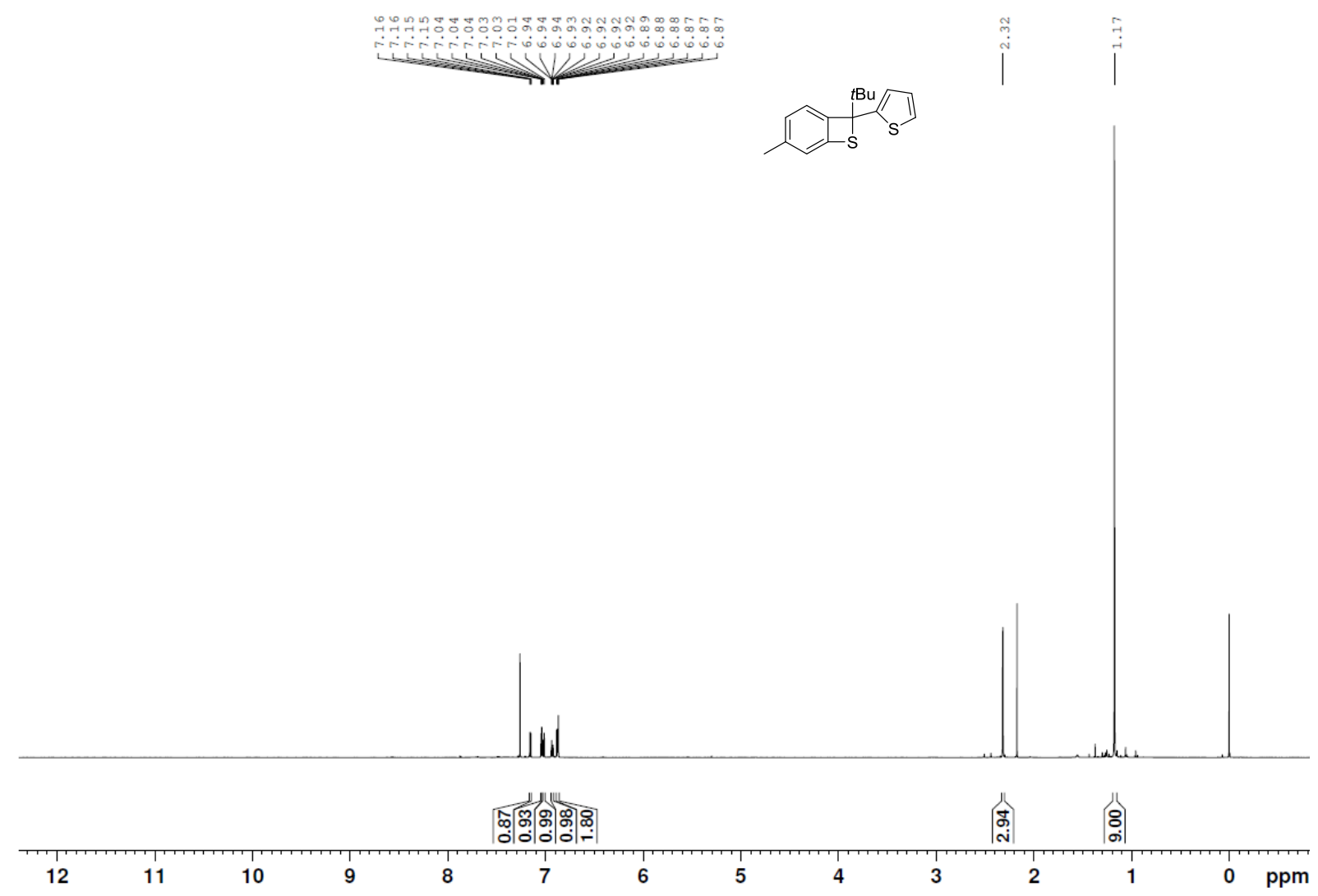

${ }^{1} \mathrm{H}-\mathrm{NMR}\left(500 \mathrm{MHz}, \mathrm{CDCl}_{3}\right)$

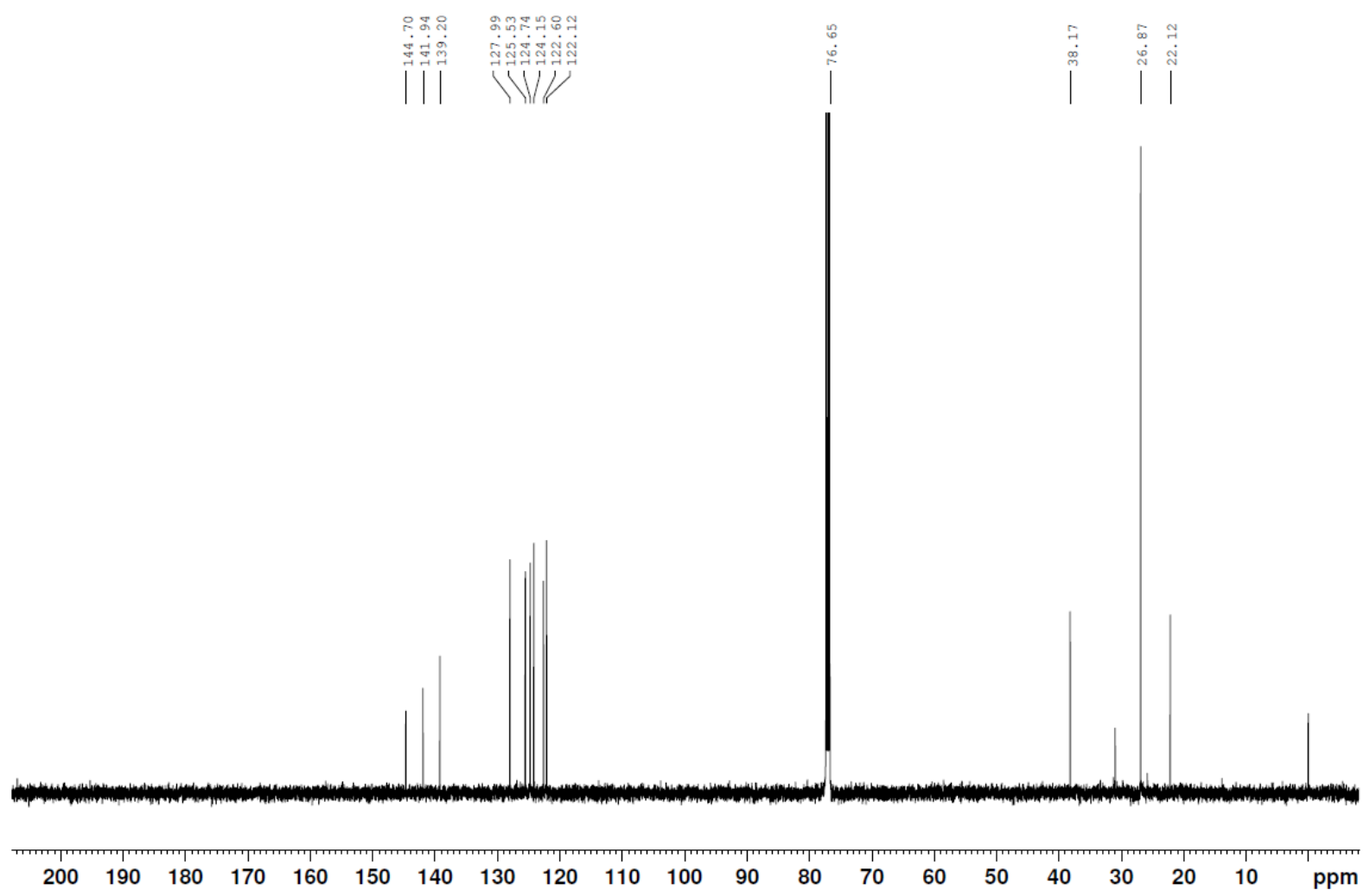


${ }^{13} \mathrm{C}-\mathrm{NMR}\left(125 \mathrm{MHz}, \mathrm{CDCl}_{3}\right)$ 
2.10.11 8-(tert-Butyl)-8-phenyl-4-(trifluoromethyl)-7-thiabicyclo[4.2.0]octa-1,3,5triene (11a)
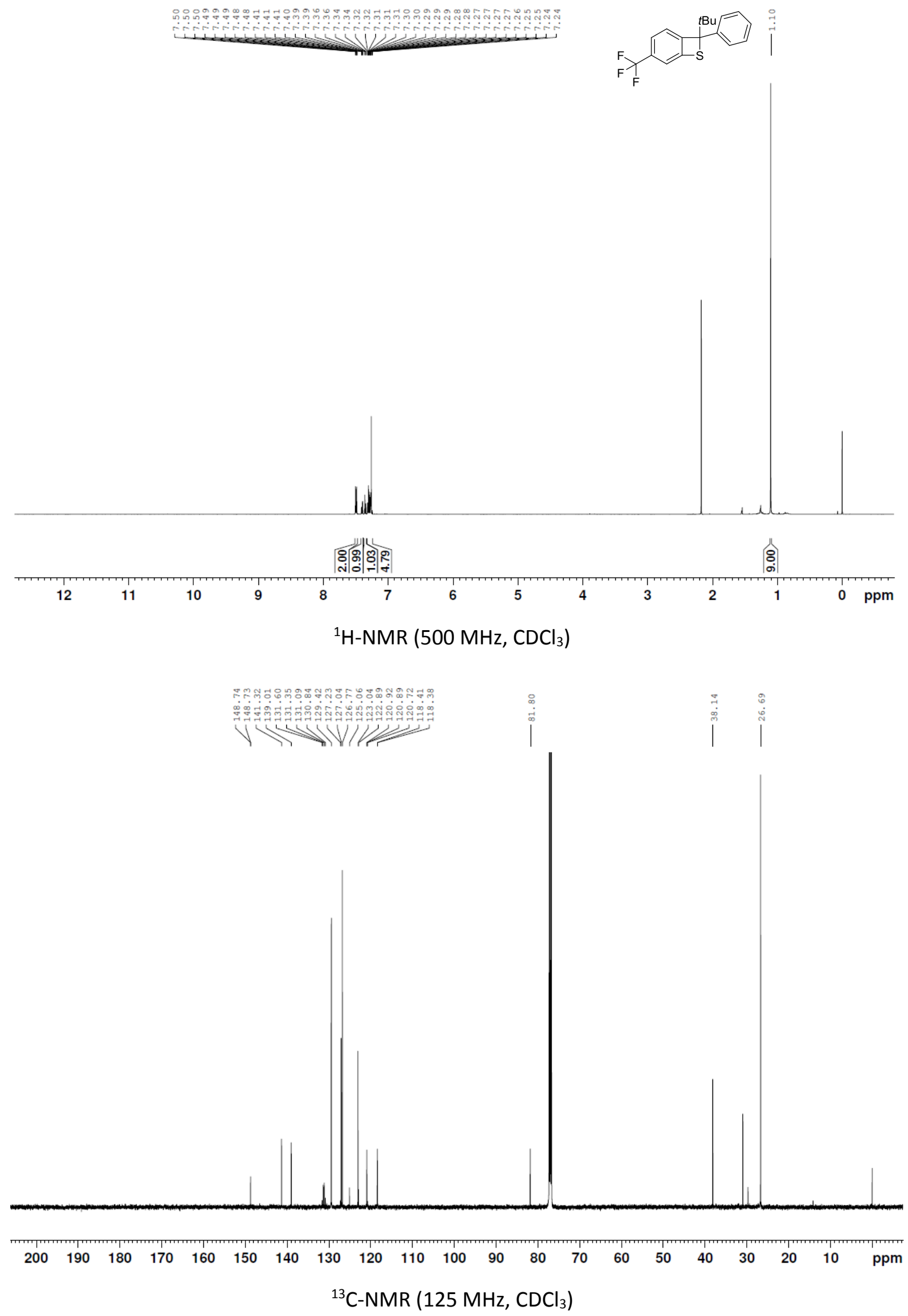


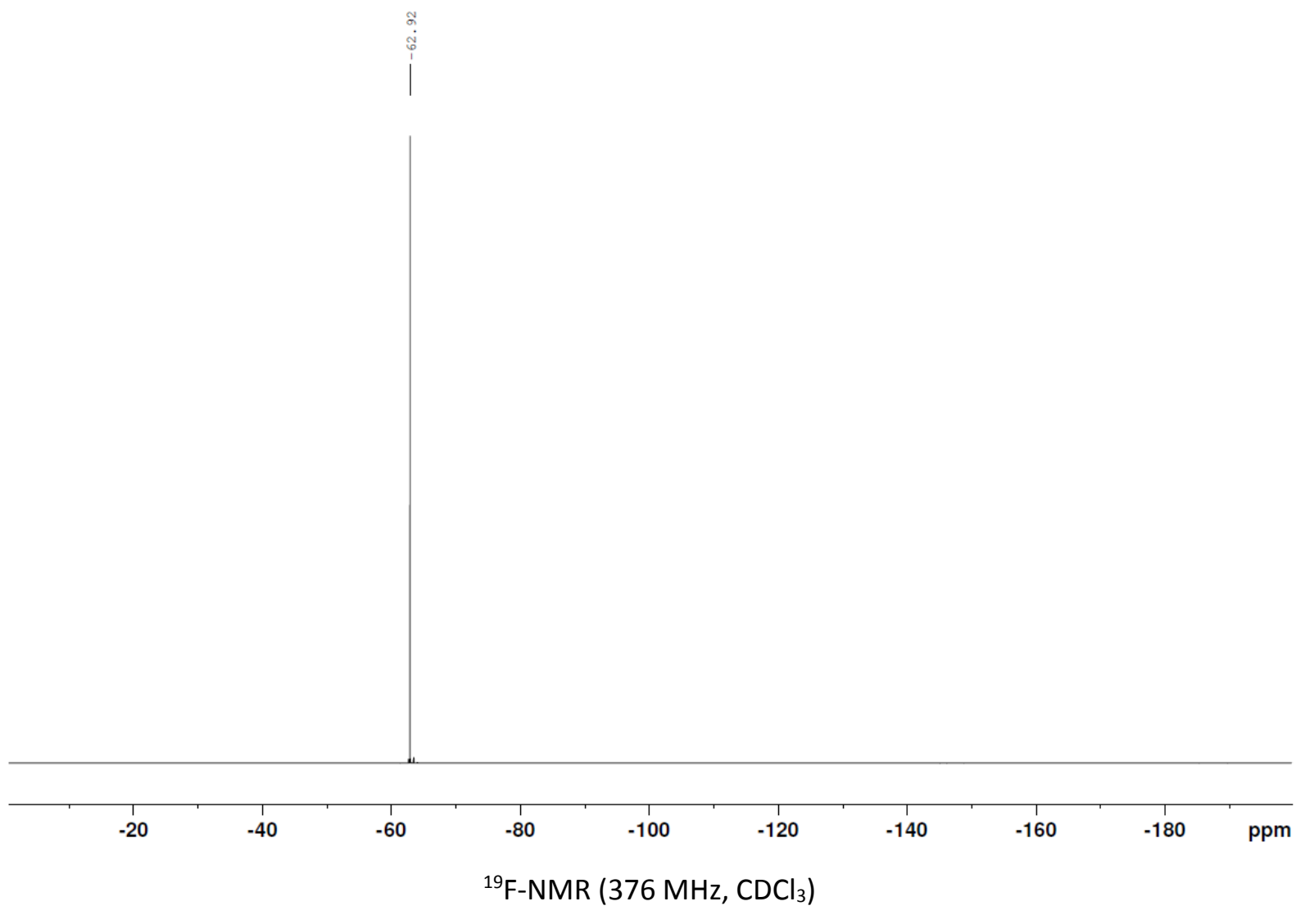


2.10.12 8-(tert-Butyl)-8-(thien-2-yl)-4-(trifluoromethyl)-7-thiabicyclo[4.2.0]octa1,3,5triene (11b)
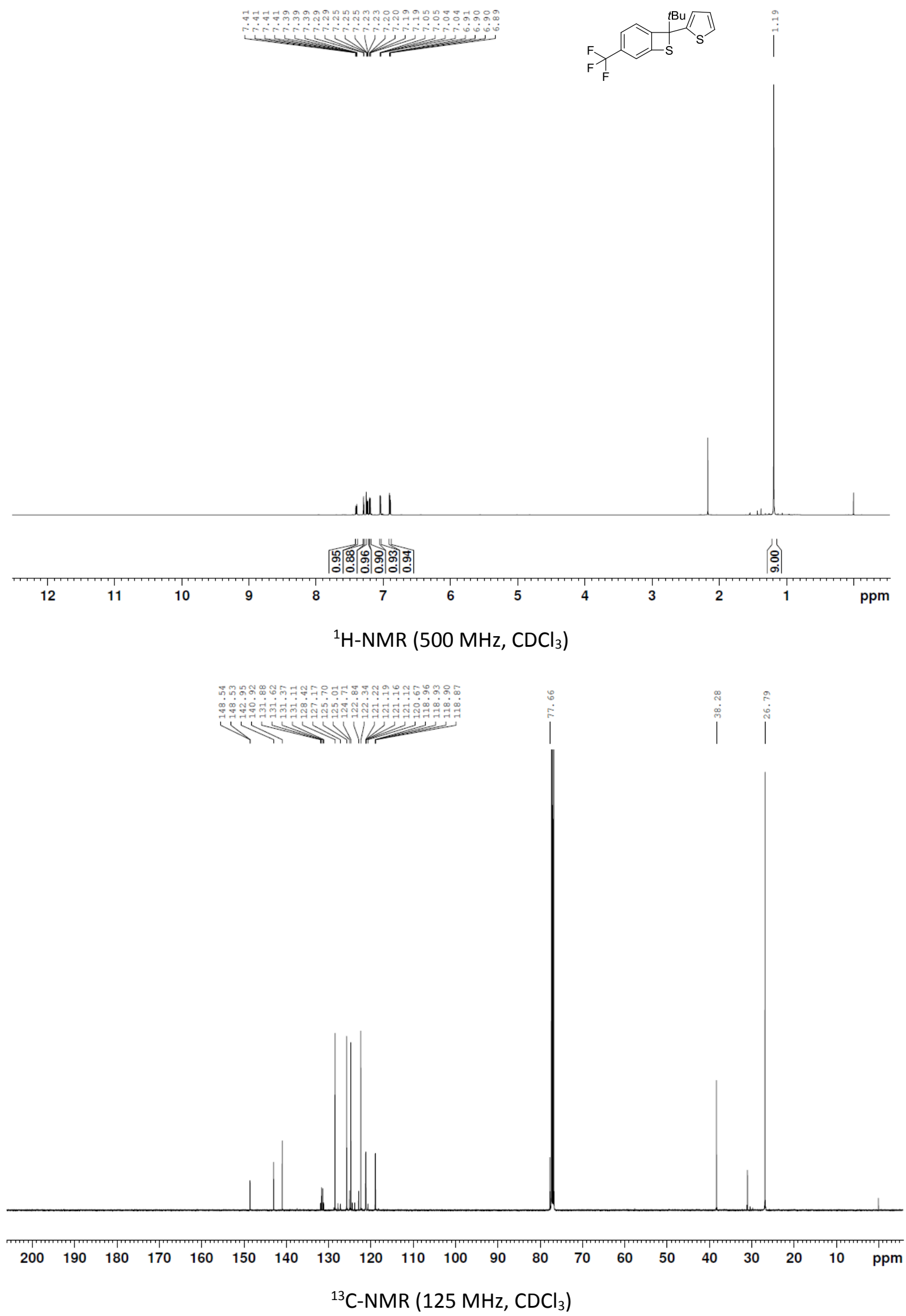


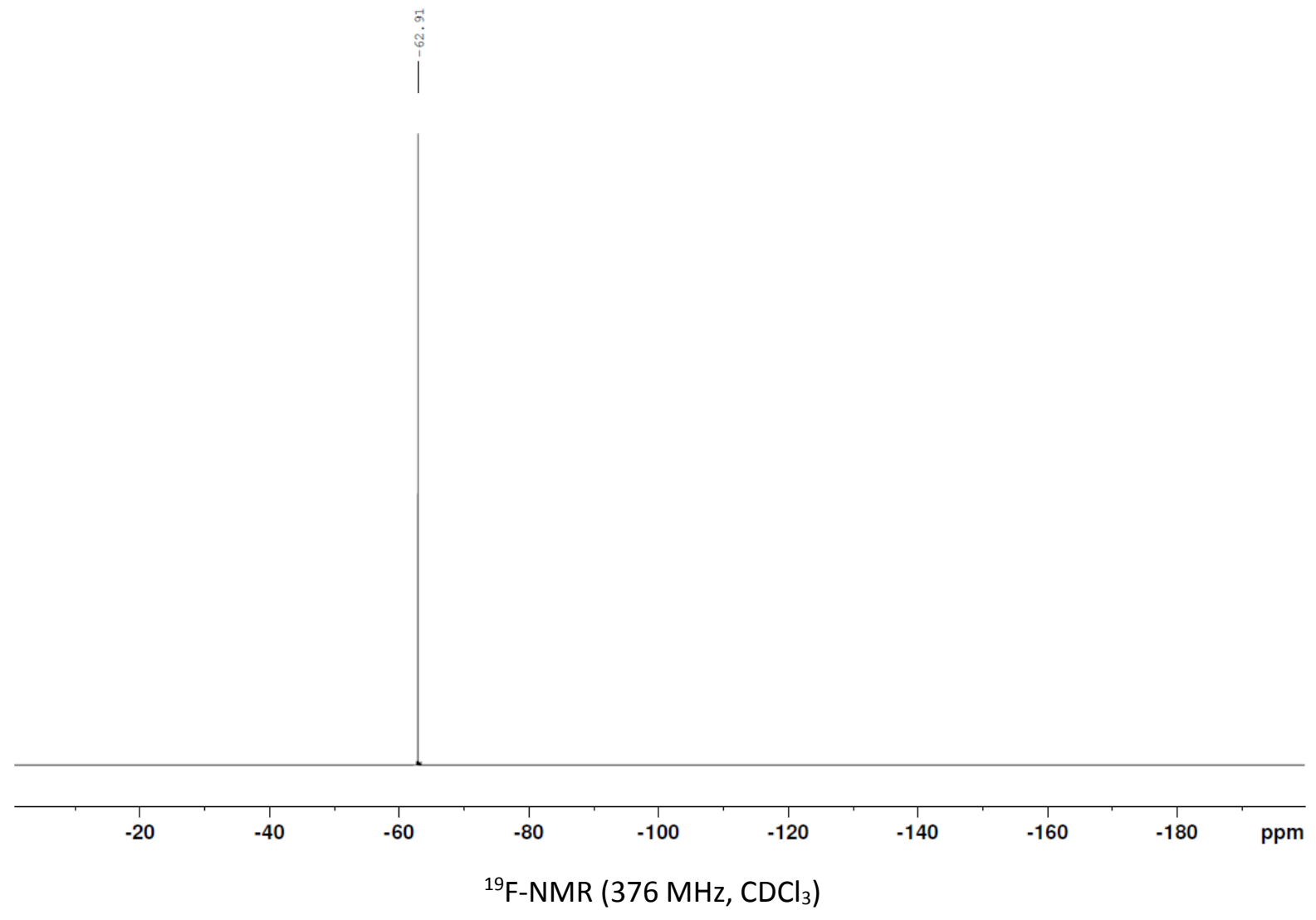




\section{Crystal Structure Determination}

\section{Preparation of a crystal of compound 10b}

The crystal used for the measurement below was obtained by dissolving compound $10 \mathrm{~b}$ (27.4 mg, $0.1 \mathrm{mmol})$ in a mixture of $\mathrm{DCM}(1.5 \mathrm{~mL})$ and $\mathrm{MeOH}(0.3 \mathrm{~mL})$. The mixture was then evaporated on a rotary evaporator without immersing the screw cap vial containing the sample into a heat source (water bath) but with rotation turned on (100 rpm). The evaporating DCM rapidly cools the sample to about $0{ }^{\circ} \mathrm{C}$. The compound $10 \mathrm{~b}$, insoluble in $\mathrm{MeOH}$, crystallized once all DCM had been evaporated. Excess $\mathrm{MeOH}$ was carefully yet quickly removed with a Pasteur pipette. The crystals were stored in a freezer prone to measurement. Although compound $\mathbf{1 0 b}$ does not incorporate solvent into the crystal cell, the crystal must never be subjected to vacuum or heat.

An array of different methods of crystallization was explored including vapor diffusion (DCM/MeOH), evaporation (DCM/MeOH) and temperature gradient methods (heptane). Only small, irregular crystals or a precipitate (powder) were formed this way.

\section{X-Ray structure determination of compound 10b}

Crystal data are summarized in Table S1. The crystal was mounted in inert oil on a nylon loop and transferred to the cold gas stream of a Rigaku-Oxford XtaLAB Synergy diffractometer using mirror-focussed Mo Ka radiation. Absorption corrections were based on multi-scans. The structure was refined anisotropically on $F^{2}$ using the program SHELXL-2018 [G. M. Sheldrick, Acta Cryst. 2015, C71, 3-8]. Hydrogen atoms were included using a riding model or rigid methyl groups.

Both thienyl groups are disordered over two positions (by exchange of the sites S2/C14 and C15/C16); occupation factors are 0.91/0.09 for molecule 1 and 0.63/0.37 for molecule 2 . Appropriate restraints were employed to improve refinement stability, but the dimensions of disordered groups should always be interpreted with caution.

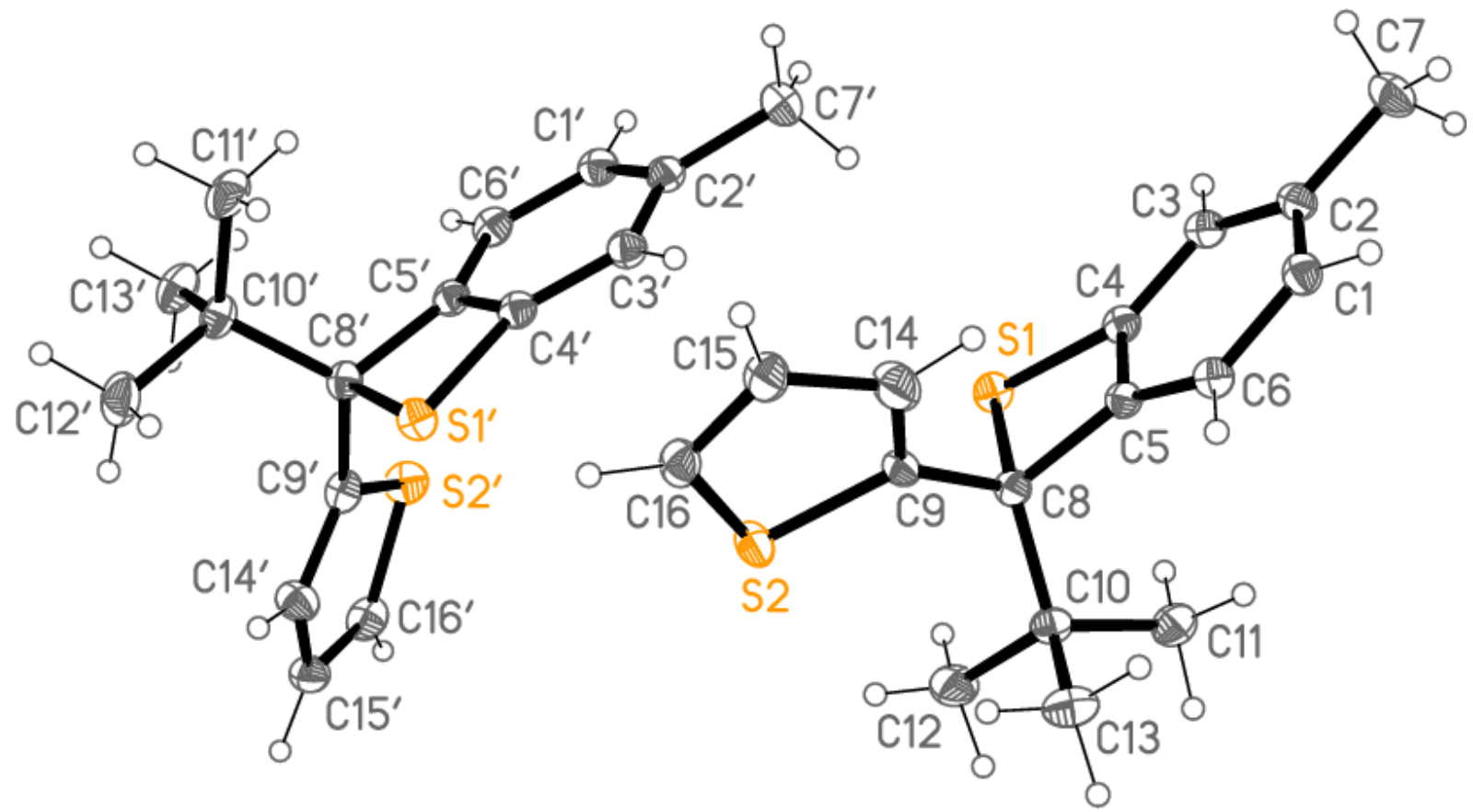

Figure S1. The structure of compound $10 \mathrm{~b}$ in the crystal. Ellipsoids represent $50 \%$ probability levels. 
Table S1. Crystallographic data for 10b

\begin{tabular}{|c|c|}
\hline Compound & $10 \mathrm{~b}$ \\
\hline CCDC number & 1993935 \\
\hline Formula & $\mathrm{C}_{16} \mathrm{H}_{18} \mathrm{~S}_{2}$ \\
\hline$M_{r}$ & 274.42 \\
\hline Temperature (K) & 101 \\
\hline Crystal habit & colourless block \\
\hline Crystal size (mm) & $0.3 \times 0.2 \times 0.1$ \\
\hline Crystal system & triclinic \\
\hline Space group & $P \overline{1}$ \\
\hline \multicolumn{2}{|l|}{ Cell dimensions: } \\
\hline$a(\AA)$ & $11.0972(3)$ \\
\hline$b(\AA ̊)$ & $11.2792(3)$ \\
\hline$c(\AA)$ & $11.4383(3)$ \\
\hline$\alpha\left({ }^{\circ}\right)$ & $89.944(2)$ \\
\hline $8\left(^{\circ}\right)$ & $85.546(2)$ \\
\hline$v\left(^{\circ}\right)$ & $86.138(2)$ \\
\hline Cell volume $\left(\AA^{3}\right)$ & 1424.13 \\
\hline Z & 4 \\
\hline$D_{x}\left(\mathrm{~g} \mathrm{~cm}^{-3}\right)$ & 1.280 \\
\hline Radiation, wavelength $(\AA ̊)$ & Mo $K \alpha, 0.71073 \AA$ \\
\hline$\mu\left(\mathrm{mm}^{-1}\right)$ & 0.35 \\
\hline $2 \Theta(\max )\left(^{\circ}\right)$ & 66 \\
\hline Reflections collected & 90183 \\
\hline Independent reflections & 10490 \\
\hline$R$ (int) & 0.048 \\
\hline Transmissions & $0.645-1.000$ \\
\hline No. of parameters & 380 \\
\hline No. of restraints & 81 \\
\hline Goodness-of-fit on $F^{2}$ & 1.04 \\
\hline$w R 2$ (all reflections) & 0.103 \\
\hline$R 1(F>4 \sigma(F))$ & 0.037 \\
\hline Max./min. $\Delta \rho\left(\mathrm{e} \AA^{-3}\right)$ & $0.59 /-0.35$ \\
\hline
\end{tabular}




\section{Computational Details}

All calculations were performed using Gaussian16 [Gaussian 16, Revision A03, M. J. Frisch, G. W. Trucks, H. B. Schlegel, G. E. Scuseria, M. A. Robb, J. R. Cheeseman, G. Scalmani, V. Barone, G. A. Petersson, H. Nakatsuji, X. Li, M. Caricato, A. Marenich, J. Bloino, B. G. Janesko, R. Gomperts, B. Mennucci, H. P. Hratchian, J. V. Ortiz, A. F. Izmaylov, J. L. Sonnenberg, D. Williams-Young, F. Ding, F. Lipparini, F. Egidi, J. Goings, B. Peng, A. Petrone, T. Henderson, D. Ranasinghe, V. G. Zakrzewski, J. Gao, N. Rega, G. Zheng, W. Liang, M. Hada, M. Ehara, K. Toyota, R. Fukuda, J. Hasegawa, M. Ishida, T. Nakajima, Y. Honda, O. Kitao, H. Nakai, T. Vreven, K. Throssell, J. A. Montgomery, Jr., J. E. Peralta, F. Ogliaro, M. Bearpark, J. J. Heyd, E. Brothers, K. N. Kudin, V. N. Staroverov, T. Keith, R. Kobayashi, J. Normand, K. Raghavachari, A. Rendell, J. C. Burant, S. S. Iyengar, J. Tomasi, M. Cossi, J. M. Millam, M. Klene, C. Adamo, R. Cammi, J. W. Ochterski, R. L. Martin, K. Morokuma, O. Farkas, J. B. Foresman, and D. J. Fox, Gaussian, Inc., Wallingford CT, 2016]. 


\subsection{D Potential Energy Scans}

The energies of the open and closed form as well as the transition state of the differently substituted benzothietes were approximated by a bong length scan (CS: $1.78535 \AA$ to $3.65535 \AA$ ). An exemplary input file and the starting geometries are given below. The following table summarizes the numerical results. The energies are also illustrated in the main text in Figure 1.

Table S2. 1D Potential Energy Scan Rbenzo: $\mathrm{NMe}_{2}$

\begin{tabular}{cccccc}
\hline $\mathrm{R}_{\text {benzo: }} \mathrm{NMe}_{2}$ & \multicolumn{5}{c}{ Energy [Hartree] } \\
\hline $\mathrm{R}_{\text {phenyl }}$ & closed & TS & open & $\Delta_{\mathrm{A}} \mathrm{U}[\mathrm{kcal} / \mathrm{mol}]$ & $\Delta_{\mathrm{R}} \mathrm{U}[\mathrm{kcal} / \mathrm{mol}]$ \\
\hline $\mathrm{NMe}_{2}$ & -1167.09 & -1167.06 & -1167.07 & 20.29 & 10.98 \\
$\mathrm{OiPr}$ & -1226.27 & -1226.24 & -1226.25 & 22.95 & 12.29 \\
$\mathrm{OMe}$ & -1147.67 & -1147.63 & -1147.65 & 23.36 & 12.46 \\
$\mathrm{H}$ & -1033.17 & -1033.13 & -1033.15 & 25.64 & 13.27 \\
$\mathrm{CF}_{3}$ & -1370.21 & -1370.16 & -1370.18 & 27.60 & 14.18 \\
$\mathrm{NO}_{2}$ & -1237.64 & -1237.60 & -1237.62 & 27.34 & 13.91 \\
$\mathrm{Br}$ & -3605.39 & -3605.35 & -3605.37 & 25.94 & 13.53
\end{tabular}

Table S3. 1D Potential Energy Scan $\mathbf{R}_{\text {benzo }}$ : OiPr

\begin{tabular}{cccccc}
\hline$R_{\text {benzo }}$ OiPr & \multicolumn{5}{c}{ Energy [Hartree] } \\
\hline $\mathrm{R}_{\text {phenyl }}$ & closed & TS & open & $\Delta_{\mathrm{A}} \mathrm{U}[\mathrm{kcal} / \mathrm{mol}]$ & $\Delta_{\mathrm{R}} \mathrm{U}[\mathrm{kcal} / \mathrm{mol}]$ \\
\hline $\mathrm{NMe}_{2}$ & -1226.27 & -1226.24 & -1226.25 & 20.67 & 13.56 \\
$\mathrm{OiPr}$ & -1285.45 & -1285.42 & -1285.43 & 23.49 & 15.16 \\
$\mathrm{OMe}$ & -1206.85 & -1206.82 & -1206.83 & 23.95 & 15.37 \\
$\mathrm{H}$ & -1092.35 & -1092.31 & -1092.33 & 26.62 & 16.44 \\
$\mathrm{CF}_{3}$ & -1429.39 & -1429.34 & -1429.36 & 28.72 & 17.25 \\
$\mathrm{NO}_{2}$ & -1296.82 & -1296.78 & -1296.80 & 29.22 & 17.39 \\
$\mathrm{Br}$ & -3664.58 & -3664.53 & -3664.55 & 26.99 & 16.70
\end{tabular}


Table S4. 1D Potential Energy Scan $\mathbf{R}_{\text {benzo }}$ : OMe

\begin{tabular}{cccccc}
\hline $\mathrm{R}_{\text {benzo: OMe }}$ & \multicolumn{5}{c}{ Energy [Hartree] } \\
\hline $\mathrm{R}_{\text {phenyl }}$ & closed & TS & open & $\Delta_{\mathrm{A}} \mathrm{U}[\mathrm{kcal} / \mathrm{mol}]$ & $\Delta_{\mathrm{R}} \mathrm{U}[\mathrm{kcal} / \mathrm{mol}]$ \\
\hline $\mathrm{NMe}_{2}$ & -1147.67 & -1147.64 & -1147.65 & 20.69 & 14.25 \\
$\mathrm{O} i \mathrm{Pr}$ & -1206.85 & -1206.82 & -1206.83 & 23.57 & 15.87 \\
$\mathrm{OMe}$ & -1128.25 & -1128.22 & -1128.23 & 24.05 & 16.13 \\
$\mathrm{H}$ & -1013.75 & -1013.71 & -1013.73 & 26.76 & 17.25 \\
$\mathrm{CF}_{3}$ & -1350.79 & -1350.74 & -1350.76 & 28.67 & 18.02 \\
$\mathrm{NO}_{2}$ & -1218.22 & -1218.18 & -1218.19 & 29.47 & 18.17 \\
$\mathrm{Br}$ & -3585.98 & -3585.93 & -3585.95 & 27.11 & 17.47
\end{tabular}

Table S5. 1D Potential Energy Scan $\mathbf{R}_{\text {benzo: }} \mathbf{H}$

\begin{tabular}{cccccc}
\hline $\mathrm{R}_{\text {benzo: }} \mathrm{H}$ & \multicolumn{5}{c}{ Energy [Hartree] } \\
\hline $\mathrm{R}_{\text {phenyl }}$ & closed & $\mathrm{TS}$ & open & $\Delta_{\mathrm{A}} \mathrm{U}[\mathrm{kcal} / \mathrm{mol}]$ & $\Delta_{\mathrm{R}} \mathrm{U}[\mathrm{kcal} / \mathrm{mol}]$ \\
\hline $\mathrm{NMe}_{2}$ & -1033.18 & -1033.14 & -1033.15 & 20.28 & 13.44 \\
$\mathrm{OiPr}$ & -1092.36 & -1092.32 & -1092.33 & 23.17 & 15.21 \\
$\mathrm{OMe}$ & -1013.76 & -1013.72 & -1013.73 & 23.26 & 15.32 \\
$\mathrm{H}$ & -899.26 & -899.21 & -899.23 & 26.05 & 16.56 \\
$\mathrm{CF}_{3}$ & -1236.29 & -1236.24 & -1236.26 & 27.97 & 17.53 \\
$\mathrm{NO}_{2}$ & -1103.73 & -1103.68 & -1103.70 & 28.89 & 17.93 \\
$\mathrm{Br}$ & -3471.48 & -3471.44 & -3471.45 & 26.44 & 16.96
\end{tabular}


Table S6. 1D Potential Energy Scan $\mathbf{R}_{\text {benzo }}: \mathbf{C F}_{3}$

\begin{tabular}{cccccc}
\hline $\mathrm{R}_{\text {benzo: }} \mathrm{CF}_{3}$ & \multicolumn{5}{c}{ Energy [Hartree] } \\
\hline $\mathrm{R}_{\text {phenyl }}$ & closed & TS & open & $\Delta_{\mathrm{A}} \mathrm{U}[\mathrm{kcal} / \mathrm{mol}]$ & $\Delta_{\mathrm{R}} \mathrm{U}[\mathrm{kcal} / \mathrm{mol}]$ \\
\hline $\mathrm{NMe}_{2}$ & -1370.21 & -1370.18 & -1370.19 & 18.78 & 12.72 \\
$\mathrm{OiPr}$ & -1429.39 & -1429.36 & -1429.37 & 21.77 & 14.91 \\
$\mathrm{OMe}$ & -1350.79 & -1350.75 & -1350.77 & 22.33 & 15.18 \\
$\mathrm{H}$ & -1236.29 & -1236.25 & -1236.26 & 25.47 & 16.66 \\
$\mathrm{CF}_{3}$ & -1573.32 & -1573.28 & -1573.29 & 27.81 & 18.07 \\
$\mathrm{NO}_{2}$ & -1440.76 & -1440.71 & -1440.73 & 28.58 & 18.25 \\
$\mathrm{Br}$ & -3808.51 & -3808.47 & -3808.48 & 25.75 & 17.03
\end{tabular}

Table S7. 1D Potential Energy Scan $\mathrm{R}_{\text {benzo }}: \mathbf{N O}_{2}$

\begin{tabular}{cccccc}
\hline $\mathrm{R}_{\text {benzo: }} \mathrm{NO}_{2}$ & \multicolumn{5}{c}{ Energy [Hartree] } \\
\hline $\mathrm{R}_{\text {phenyl }}$ & closed & $\mathrm{TS}$ & open & $\Delta_{\mathrm{A}} \mathrm{U}[\mathrm{kcal} / \mathrm{mol}]$ & $\Delta_{\mathrm{R}} \mathrm{U}[\mathrm{kcal} / \mathrm{mol}]$ \\
\hline $\mathrm{NMe}_{2}$ & -1237.65 & -1237.62 & -1237.63 & 16.99 & 12.39 \\
$\mathrm{OiPr}$ & -1296.83 & -1296.80 & -1296.80 & 20.58 & 15.07 \\
$\mathrm{OMe}$ & -1218.23 & -1218.19 & -1218.20 & 21.61 & 15.57 \\
$\mathrm{H}$ & -1103.73 & -1103.69 & -1103.70 & 24.65 & 17.46 \\
$\mathrm{CF}_{3}$ & -1440.76 & -1440.72 & -1440.73 & 26.90 & 18.75 \\
$\mathrm{NO}_{2}$ & -1308.19 & -1308.15 & -1308.16 & 28.01 & 19.32 \\
$\mathrm{Br}$ & -3675.95 & -3675.91 & -3675.92 & 24.95 & 17.80
\end{tabular}


Table S8. 1D Potential Energy Scan $\mathbf{R}_{\text {benzo }}$ : $\mathrm{Br}$

\begin{tabular}{cccccc}
\hline $\mathrm{R}_{\text {benzo: }} \mathrm{Br}$ & \multicolumn{5}{c}{ Energy [Hartree] } \\
\hline $\mathrm{R}_{\text {phenyl }}$ & closed & $\mathrm{TS}$ & open & $\Delta_{\mathrm{A}} \mathrm{U}[\mathrm{kcal} / \mathrm{mol}]$ & $\Delta_{\mathrm{R}} \mathrm{U}[\mathrm{kcal} / \mathrm{mol}]$ \\
\hline $\mathrm{NMe}_{2}$ & -3605.40 & -3605.37 & -3605.38 & 19.26 & 12.94 \\
$\mathrm{OiPr}$ & -3664.58 & -3664.54 & -3664.55 & 22.37 & 14.84 \\
$\mathrm{OMe}$ & -3585.98 & -3585.94 & -3585.95 & 22.92 & 15.14 \\
$\mathrm{H}$ & -3471.48 & -3471.44 & -3471.45 & 25.81 & 16.43 \\
$\mathrm{CF}_{3}$ & -3808.51 & -3808.46 & -3808.48 & 27.86 & 17.34 \\
$\mathrm{NO}_{2}$ & -3675.94 & -3675.90 & -3675.92 & 28.81 & 17.73 \\
$\mathrm{Br}$ & -6043.70 & -6043.66 & -6043.67 & 26.17 & 16.72
\end{tabular}




\subsection{Methods and Geometries}

The substitution pattern of the benzothiete is encoded in the file name. The file name follows the structure " $<R_{\text {benzo }}><R_{\text {phenyl }}>$ ". All species were built from the optimized open structure of D4 and underwent a relaxed PES scan along the carbon-sulfur bond.

Table S9. Input File Topology

\begin{tabular}{|c|c|c|c|c|c|c|c|}
\hline \multirow[b]{2}{*}{ Functional group } & \multicolumn{7}{|c|}{$\mathbf{R}_{\text {benzo }}$} \\
\hline & (A) $\mathrm{NMe}_{2}$ & (B) OiPr & (C) OMe & (D) $\mathrm{H}$ & (E) $\mathrm{CF}_{3}$ & (F) $\mathrm{NO}_{2}$ & (G) $\mathrm{Br}$ \\
\hline (1) $\mathrm{NMe}_{2}$ & $\mathrm{~A} 1$ & B1 & $\mathrm{C} 1$ & D1 & E1 & $\mathrm{F} 1$ & G1 \\
\hline (2) OiPr & $\mathrm{A} 2$ & B2 & $\mathrm{C} 2$ & D2 & E2 & $\mathrm{F} 2$ & G2 \\
\hline (3) OMe & A3 & B3 & $\mathrm{C} 3$ & D3 & E3 & F3 & G3 \\
\hline (4) $\mathrm{H}$ & A4 & B4 & $\mathrm{C} 4$ & D4 & E4 & $\mathrm{F} 4$ & G4 \\
\hline (5) $\mathrm{CF}_{3}$ & A5 & B5 & C5 & D5 & E5 & F5 & G5 \\
\hline (6) $\mathrm{NO}_{2}$ & A6 & $\mathrm{B} 6$ & $\mathrm{C} 6$ & D6 & E6 & F6 & G6 \\
\hline (7) $\mathrm{Br}$ & A7 & $\mathrm{B} 7$ & $\mathrm{C7}$ & D7 & E7 & $\mathrm{F7}$ & G7 \\
\hline
\end{tabular}

\subsubsection{Scans}

All relaxed PES scans of the carbon-sulfur bond were performed with the following protocols.

\subsubsection{Exemplary Scan Input File}

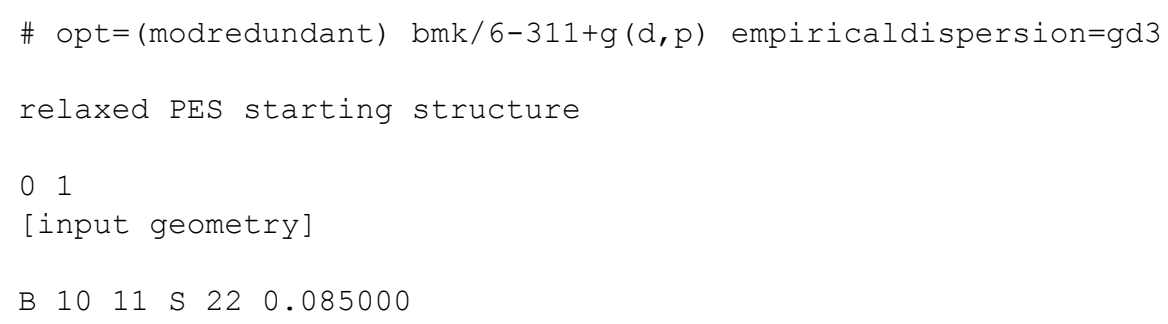

\subsubsection{Transition states}

After performing scans of each variant, the transition states for the most electron withdrawing and electron donating functional groups were optimized to compare molecular orbitals and electron densities.

\subsubsection{Exemplary QST3 Input File}

\# opt $=(\mathrm{QST} 3) \quad b m k / 6-311+\mathrm{g}(\mathrm{d}, \mathrm{p})$ freq empiricaldispersion=gd3 


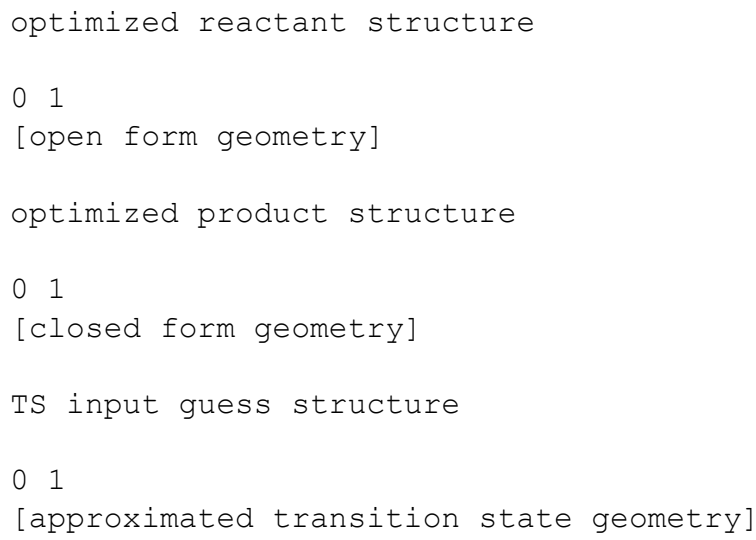

\subsubsection{Transition States Geometries}

$\begin{array}{lr}\text { Oxygen-D4-(H, H }) & -3.32763900 \\ \mathrm{C} & -1.94601500 \\ \mathrm{C} & -1.17903600 \\ \mathrm{C} & -1.64489000 \\ \mathrm{C} & -2.98563100 \\ \mathrm{C} & -3.79993300 \\ \mathrm{C} & -3.95943100 \\ \mathrm{H} & -1.01443400 \\ \mathrm{H} & -3.42086600 \\ \mathrm{H} & -4.83995500 \\ \mathrm{H} & 0.04670200 \\ \mathrm{C} & 1.37385400 \\ \mathrm{C} & 1.54911200 \\ \mathrm{C} & 2.50690300 \\ \mathrm{C} & 2.83009100 \\ \mathrm{C} & 0.67320900 \\ \mathrm{H} & 3.78559400 \\ \mathrm{C} & 2.36883100 \\ \mathrm{H} & 3.94592200 \\ \mathrm{C} & 2.96532400 \\ \mathrm{H} & 4.65595000 \\ \mathrm{H} & 4.94458900 \\ \mathrm{H} & -1.16135200 \\ \mathrm{O} & -0.01261300 \\ \mathrm{H} & \end{array}$

$\begin{array}{rr}0.71879200 & 0.51131800 \\ 0.94655600 & 0.30691100 \\ -0.00215900 & -0.40423300 \\ -1.27554600 & -0.73465200 \\ -1.53409700 & -0.44832100 \\ -0.54097800 & 0.15256100 \\ 1.45639900 & 0.99386500 \\ -2.01148200 & -1.22499200 \\ -2.49362400 & -0.70476200 \\ -0.78395200 & 0.35307800 \\ 0.76848000 & -0.56603400 \\ 0.29275000 & -0.24152600 \\ -0.88097800 & 0.52150300 \\ 1.01238800 & -0.67702900 \\ -1.33868400 & 0.81181700 \\ -1.40804300 & 0.88645400 \\ 0.55376800 & -0.38080500 \\ 1.92632100 & -1.24787000 \\ -0.62337800 & 0.36065900 \\ -2.24182800 & 1.39778300 \\ 1.10581600 & -0.71977300 \\ -0.97922400 & 0.59546000 \\ 1.89688400 & 0.69554400 \\ 1.67305200 & -1.16660400\end{array}$

Sulphur-A1- $\left(\mathrm{NMe}_{2}, \mathrm{NMe}_{2}\right)$

$\begin{array}{rrr}3.20223500 & -0.85862800 & 0.22223700 \\ 1.87475700 & -0.49947400 & 0.44872800 \\ 1.49747300 & 0.85648100 & 0.30975000 \\ 2.38692900 & 1.83120600 & -0.18984900 \\ 3.74515100 & 1.47486100 & -0.34869000 \\ 4.12761900 & 0.16189800 & -0.14822900 \\ 1.14016600 & -1.21904600 & 0.78879400 \\ 4.46679600 & 2.22420600 & -0.65865500 \\ 5.17269700 & -0.10899500 & -0.26169500 \\ 1.57828500 & 3.30593200 & -0.64858000 \\ 0.22616000 & 1.44108900 & 0.67183100 \\ -1.05257600 & 0.86430000 & 0.42743500 \\ -1.24238100 & -0.17595700 & -0.51779300 \\ -2.20337400 & 1.34581200 & 1.10106900 \\ -2.48379300 & -0.73049300 & -0.74569300 \\ -0.38757300 & -0.52433200 & -1.08870300 \\ -3.45302300 & 0.80407300 & 0.88818600 \\ -2.09336900 & 2.16377500 & 1.80811200 \\ -3.63369300 & -0.26345900 & -0.04142200\end{array}$




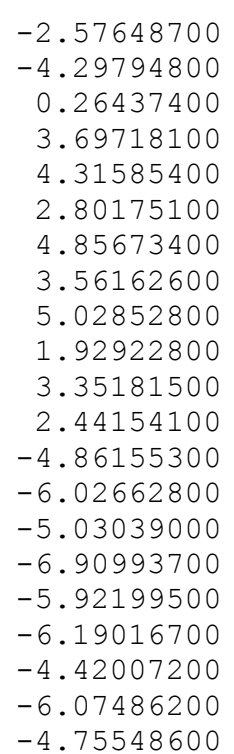

$-4.75548600$

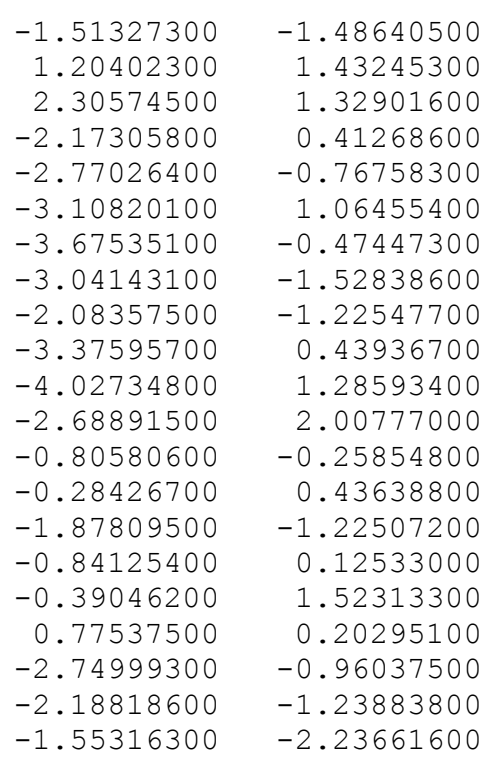

$-0.84930700$

$-0.51478600$

0.83815700

1.84973000

1.53061600

0.21307800

$-1.25919400$

2. 30102400

$-0.01705200$

3. 27222400

1. 42406700

0.82317100

$-0.18668600$

1.27067900

$-0.76292400$

$-0.49843300$

0.70199500

2.06618500

$-0.31498100$

$-1.53841400$

1.02339900

2.26906200

$-2.15158400$

$-2.50160100$

$-3.21133100$

$-3.57497900$

$-2.24864900$

$-1.99545000$

$-3.25636600$

$-4.16954000$

$-3.07520500$

$-0.92797900$

$-0.50031800$

$-1.83521800$

0.17663200

0.36824600

0.29290500

$-0.13294700$

$-0.23516700$

$-0.10491000$

0.65276600

$-0.48671100$

$-0.23370200$

$-0.63619000$

0.63575100

0.39236100

$-0.58167000$

1.11395400

$-0.79891300$

$-1.17650400$

0.90648700

1.84394300

$-0.04317700$

$-1.54006100$

1. 45803100

1. 31613400

0.30381200

$-0.19082700$

0.40705900

$-0.07212400$

$-1.25422300$

0.38257000

$-0.47429100$

0.50804900

1.29669600

$-0.26328000$

0.38879600

$-1.08379100$
Sulphur-D4-(H, H)

$\begin{array}{ll}\text { C } & -2.43133500 \\ \text { C } & -1.15854700 \\ \text { C } & -1.01565600 \\ \text { C } & -2.12068500 \\ \text { C } & -3.41967400 \\ \text { C } & -3.55411500 \\ \text { H } & -0.29621000 \\ \text { H } & -4.27812900 \\ \text { H } & -4.54320700\end{array}$

-2.13980200
-1.60258200
-0.19891200
0.63083800
0.07607300
-1.29572500
-2.22953100
0.71134200
-1.74185000

$-0.29385800$

$-0.38774700$

$-0.29674700$

0.04416100

0.05335000

$-0.09620400$

$-0.59172300$

0.24573200

$-0.04370700$ 


$$
\begin{array}{r}
-1.60617000 \\
0.15222100 \\
1.50867900 \\
1.78136200 \\
2.58797700 \\
3.09351700 \\
0.95620400 \\
3.89818000 \\
2.38184000 \\
4.15171500 \\
3.29871700 \\
4.72196500 \\
0.01967300 \\
5.17488700 \\
-2.57886700
\end{array}
$$

Sulphur-F1- $\left(\mathrm{NO}_{2}, \mathrm{NMe}_{2}\right)$

$\begin{array}{lr}\text { C } & -3.18435800 \\ \mathrm{C} & -1.87255300 \\ \mathrm{C} & -1.53994300 \\ \mathrm{C} & -2.45886000 \\ \mathrm{C} & -3.80225800 \\ \mathrm{C} & -4.15315800 \\ \mathrm{H} & -1.16296100 \\ \mathrm{H} & -4.52966100 \\ \mathrm{H} & -5.16203800 \\ \mathrm{~S} & -1.71540500 \\ \mathrm{C} & -0.28048600 \\ \mathrm{C} & 1.00246300 \\ \mathrm{C} & 1.22408100 \\ \mathrm{C} & 2.14169600 \\ \mathrm{C} & 2.47573800 \\ \mathrm{H} & 0.38556400 \\ \mathrm{C} & 3.40167000 \\ \mathrm{H} & 2.00570200 \\ \mathrm{C} & 3.61306000 \\ \mathrm{H} & 2.58912100 \\ \mathrm{H} & 4.23218300 \\ \mathrm{H} & -0.35327100 \\ \mathrm{~N} & 4.84711300 \\ \mathrm{C} & 6.00233000 \\ \mathrm{C} & 5.04674700 \\ \mathrm{H} & 6.89835200 \\ \mathrm{H} & 5.90482900 \\ \mathrm{H} & 6.13365300 \\ \mathrm{H} & 4.44553100 \\ \mathrm{H} & 6.09544600 \\ \mathrm{H} & 4.78233600 \\ \mathrm{~N} & -3.56609900 \\ \mathrm{O} & -2.69674100 \\ \mathrm{O} & -4.73856200\end{array}$

Sulphur-F6- $\left(\mathrm{NO}_{2}, \mathrm{NO}_{2}\right)$

$\begin{array}{lr}\text { C } & 3.07567300 \\ \mathrm{C} & 1.74799900 \\ \mathrm{C} & 1.42980000 \\ \mathrm{C} & 2.39757100 \\ \mathrm{C} & 3.76098200 \\ \mathrm{C} & 4.08626100 \\ \mathrm{H} & 1.01359500 \\ \mathrm{H} & 4.51802900 \\ \mathrm{H} & 5.10761800 \\ \mathrm{~S} & 1.66698000 \\ \mathrm{C} & 0.19215300 \\ \mathrm{C} & -1.12258200 \\ \mathrm{C} & -1.34144200 \\ \mathrm{C} & -2.21405200 \\ \mathrm{C} & -2.60833600\end{array}$

\author{
2.20847600 \\ 0.57837900 \\ 0.20586600 \\ $-0.73481000$ \\ 0.80424200 \\ $-1.09050500$ \\ $-1.15463100$ \\ 0.44327000 \\ 1.54519700 \\ $-0.50754500$ \\ $-1.80820900$ \\ 0.89998800 \\ 1.42364600 \\ $-0.78496800$ \\ $-3.20933500$ \\ 0.54068800 \\ $-0.58379700$ \\ $-0.25530900$ \\ 0.76265700 \\ $-0.94271400$ \\ 1.05481000 \\ 1.32865800 \\ $-0.64932200$ \\ $-1.71010700$ \\ 0.34702400 \\ 1.84251100 \\ $-1.18808300$ \\ $-1.25302900$ \\ 0.58144300 \\ $-0.40052400$
}

$$
\begin{array}{rr}
-0.96891400 & -0.11196400 \\
-0.60331600 & -0.40343100 \\
0.74690000 & -0.28842100 \\
1.70530000 & 0.22154100 \\
1.30836300 & 0.43543500 \\
-0.02009300 & 0.28419500 \\
-1.34660300 & -0.74718400 \\
2.03956300 & 0.77179800 \\
-0.36383600 & 0.48103500 \\
3.21668200 & 0.60515400 \\
1.34889700 & -0.69379200 \\
0.82501000 & -0.43014100 \\
-0.20795200 & 0.52410400 \\
1.33601800 & -1.11296800 \\
-0.72992800 & 0.74535500 \\
-0.57788100 & 1.10506600 \\
0.82978300 & -0.90292800 \\
2.14666900 & -1.82382400 \\
-0.23634300 & 0.02942700 \\
-1.50913100 & 1.48644000 \\
1.24749900 & -1.45550700 \\
2.18453700 & -1.38540200 \\
-0.74852200 & 0.23714900 \\
-0.19954900 & -0.45931700 \\
-1.83886000 & 1.18256200 \\
-0.73009500 & -0.13986900 \\
-0.31641200 & -1.54511300 \\
0.86419600 & -0.22843400 \\
-2.71297400 & 0.90806000 \\
-2.13280900 & 1.17431800 \\
-1.53373900 & 2.20217800 \\
-2.36082800 & -0.23892300 \\
-3.17729000 & -0.54849100 \\
-2.66425100 & -0.02545200
\end{array}
$$

$$
\begin{array}{rr}
-0.97151400 & 0.09435700 \\
-0.63249100 & 0.28214500 \\
0.73672500 & 0.23832800 \\
1.71224800 & -0.15539400 \\
1.33213400 & -0.24517600 \\
-0.00423100 & -0.14356800 \\
-1.39415000 & 0.51554300 \\
2.07688400 & -0.46531200 \\
-0.34713200 & -0.26085200 \\
3.20150700 & -0.59259700 \\
1.34215500 & 0.62150100 \\
0.79025500 & 0.38058500 \\
-0.16917300 & -0.63334700 \\
1.23550300 & 1.15956500 \\
-0.69964500 & -0.83835200
\end{array}
$$




$\begin{array}{rrr}-0.51970300 & -0.47308700 & -1.27236500 \\ -3.48410600 & 0.71104600 & 0.96446300 \\ -2.05363400 & 1.98911900 & 1.92445700 \\ -3.65405500 & -0.25264100 & -0.03023000 \\ -2.80394500 & -1.43501900 & -1.60923700 \\ -4.33401100 & 1.02799400 & 1.55611100 \\ 0.25287700 & 2.18870700 & 1.29853700 \\ -5.00281700 & -0.81097100 & -0.24809300 \\ -5.90477600 & -0.40800900 & 0.47395400 \\ -5.13399600 & -1.64237200 & -1.13567300 \\ 3.45620500 & -2.37840700 & 0.18066300 \\ 2.56710600 & -3.20507500 & 0.36568600 \\ 4.64398600 & -2.65879500 & 0.05821000\end{array}$




\subsubsection{Optimizations}

The Oxygen-D4-(H, H) and Sulfur(A1, A2, D4, F1, F6) were optimized according to the protocol below. The calculated energies are summarized in the following table.

\subsubsection{Exemplary Transition State Optimization Input File}

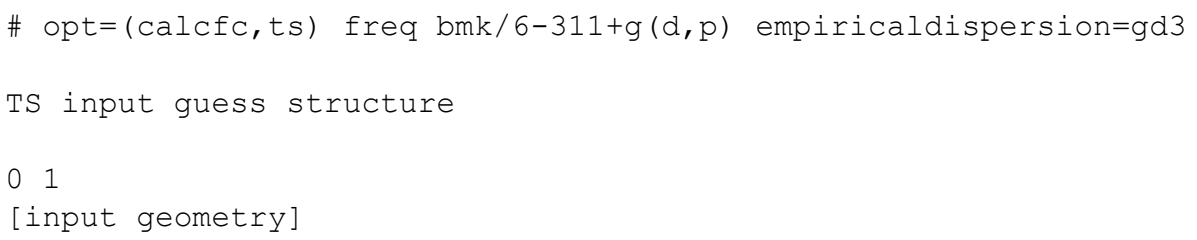

\subsubsection{Exemplary Optimization Input File}

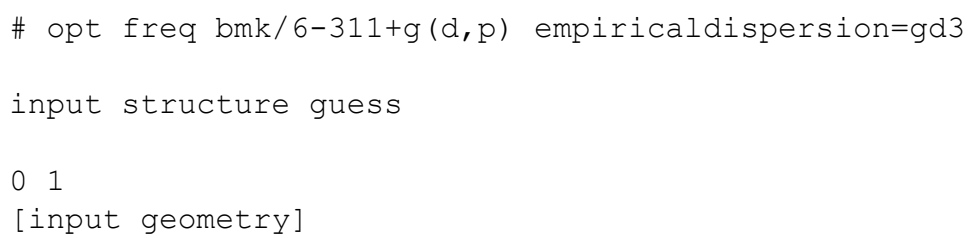

\subsubsection{Open and Closed Geometries}

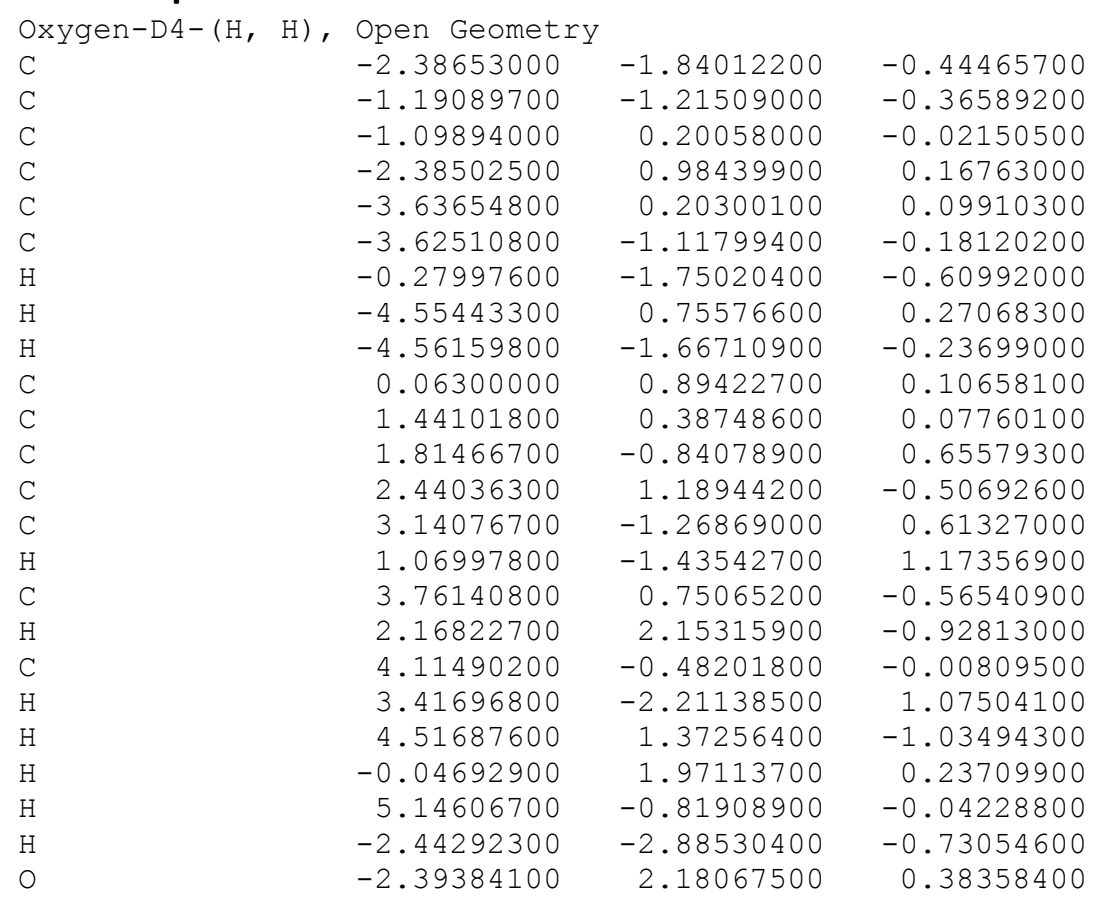




$\begin{array}{lrrr}\text { Oxygen-D4-(H, H }), \text { Closed Geometry } \\ \text { C } & 3.16339700 & -1.30834800 & \\ \text { C } & 1.91412300 & -1.04464800 & 1.199386700 \\ \text { C } & 1.23337900 & 0.03551200 & 0.67992200 \\ \text { C } & 1.73153200 & 0.79880400 & -0.36581100 \\ \text { C } & 2.94739400 & 0.58143300 & -0.98548300 \\ \text { C } & 3.65477000 & -0.52040200 & -0.45668900 \\ \text { H } & 1.53852700 & -1.66068700 & 2.00929600 \\ \text { H } & 3.33250000 & 1.18312000 & -1.79995600 \\ \text { H } & 4.62096800 & -0.76945600 & -0.88485400 \\ \text { C } & 0.01317700 & 0.95907700 & 0.64095200 \\ \text { C } & -1.31759100 & 0.37595900 & 0.24347800 \\ \text { C } & -1.42692400 & -0.36456500 & -0.94108500 \\ \text { C } & -2.44089000 & 0.55349400 & 1.05420000 \\ \text { C } & -2.65000700 & -0.92016700 & -1.30767700 \\ \text { H } & -0.54920700 & -0.49206800 & -1.56789600 \\ \text { C } & -3.66773200 & -0.01362100 & 0.69199200 \\ \text { H } & -2.36131700 & 1.13720400 & 1.96732600 \\ \text { C } & -3.77326800 & -0.74870900 & -0.48866100 \\ \text { H } & -2.73175000 & -1.48838900 & -2.22888600 \\ \text { H } & -4.53684800 & 0.12728500 & 1.32671600 \\ \text { H } & -0.07432400 & 1.64080700 & 1.49151400 \\ \text { H } & -4.72539600 & -1.18453200 & -0.77460100 \\ \text { H } & 3.76007900 & -2.14238400 & 0.94796200 \\ \text { O } & 0.67982500 & 1.66827400 & -0.47952100\end{array}$

Sulphur-A1- $\left(\mathrm{NMe}_{2}, \mathrm{NMe}_{2}\right)$, Open Geometry

$\begin{array}{lrrr}\mathrm{C} & 2.91213800 & 1.15204600 & -0.22070600 \\ \mathrm{C} & 1.68971900 & 0.54408700 & -0.17861900 \\ \mathrm{C} & 1.53982600 & -0.89751500 & -0.04488100 \\ \mathrm{C} & 2.75264000 & -1.73899400 & 0.08469600 \\ \mathrm{C} & 4.01784700 & -1.03320100 & -0.00665200 \\ \mathrm{C} & 4.09574600 & 0.31401000 & -0.13269400 \\ \mathrm{H} & 0.79262400 & 1.12257600 & -0.33892900 \\ \mathrm{H} & 4.92074200 & -1.63332400 & 0.03037700 \\ \mathrm{H} & 5.06993400 & 0.78318800 & -0.22848600 \\ \mathrm{~S} & 2.76311000 & -3.39545600 & 0.37389100 \\ \mathrm{C} & 0.30711200 & -1.51602400 & -0.09803200 \\ \mathrm{C} & -1.02394800 & -0.94874000 & -0.03768000 \\ \mathrm{C} & -1.36360500 & 0.23541500 & 0.65552800 \\ \mathrm{C} & -2.09150000 & -1.65755900 & -0.63496700 \\ \mathrm{C} & -2.66266800 & 0.71225200 & 0.69960200 \\ \mathrm{H} & -0.60003500 & 0.76025800 & 1.21848400 \\ \mathrm{C} & -3.39264800 & -1.18584100 & -0.62276400 \\ \mathrm{H} & -1.88080400 & -2.59892800 & -1.13548900 \\ \mathrm{C} & -3.71865200 & 0.03261600 & 0.03247800 \\ \mathrm{H} & -2.86305600 & 1.60617900 & 1.27507900 \\ \mathrm{H} & -4.15951400 & -1.77050200 & -1.11305400 \\ \mathrm{H} & 0.34164800 & -2.59973600 & -0.20369500 \\ \mathrm{~N} & 3.11201700 & 2.52408200 & -0.45714300 \\ \mathrm{C} & 3.94404400 & 3.21557200 & 0.52407900 \\ \mathrm{C} & 1.93646300 & 3.30002700 & -0.79978300 \\ \mathrm{H} & 4.24850700 & 4.18583300 & 0.12112100 \\ \mathrm{H} & 3.39874600 & 3.38049000 & 1.46954700 \\ \mathrm{H} & 4.84547700 & 2.64437800 & 0.74846100 \\ \mathrm{H} & 1.22256600 & 3.38129500 & 0.04012900 \\ \mathrm{H} & 2.24977800 & 4.30953100 & -1.07911000 \\ \mathrm{H} & 1.42382700 & 2.84984400 & -1.65390300 \\ \mathrm{~N} & -4.99709200 & 0.52010600 & 0.03894400 \\ \mathrm{C} & -6.07486500 & -0.26145500 & -0.53862600 \\ \mathrm{C} & -5.31652300 & 1.71333300 & 0.80004000 \\ \mathrm{H} & -7.00973800 & 0.29143100 & -0.44621800 \\ \mathrm{H} & -5.89913900 & -0.44887300 & -1.60464200 \\ \mathrm{H} & -6.19482300 & -1.22892200 & -0.03129600 \\ \mathrm{H} & -4.71191100 & 2.56519600 & 0.46722100 \\ \mathrm{H} & -6.36520400 & 1.96816900 & 0.64620800 \\ \mathrm{H} & -5.15062200 & 1.56973400 & 1.87721700 \\ & & & \\ & & & \\ & & & \end{array}$




\begin{tabular}{|c|c|c|c|}
\hline C & -3.38128700 & 0.60017500 & 0.10368300 \\
\hline C & -2.13334600 & 0.34856200 & 0.71891200 \\
\hline $\mathrm{C}$ & -1.51905400 & -0.86429200 & 0.44260800 \\
\hline C & -2.06130700 & -1.79518600 & -0.42875800 \\
\hline $\mathrm{C}$ & -3.28891600 & -1.58588400 & -1.04732500 \\
\hline $\mathrm{C}$ & -3.93522100 & -0.38083400 & -0.75384500 \\
\hline $\mathrm{H}$ & -1.65904100 & 1.07066900 & 1.37204100 \\
\hline $\mathrm{H}$ & -3.74208500 & -2.31027100 & -1.71502900 \\
\hline $\mathrm{H}$ & -4.91327100 & -0.19751300 & -1.18550300 \\
\hline S & -0.71679700 & -2.99398000 & -0.34204000 \\
\hline C & -0.22991000 & -1.55758500 & 0.84486600 \\
\hline $\mathrm{C}$ & 1.06483200 & -0.86251700 & 0.55135300 \\
\hline C & 1.23792900 & -0.12874000 & -0.63039500 \\
\hline $\mathrm{C}$ & 2.14547900 & -0.93640200 & 1.43333800 \\
\hline C & 2.43373500 & 0.51418200 & -0.91832600 \\
\hline $\mathrm{H}$ & 0.41643300 & -0.07201400 & -1.33975200 \\
\hline C & 3.35539700 & -0.29713700 & 1.16510200 \\
\hline $\mathrm{H}$ & 2.04891300 & -1.50714700 & 2.35362200 \\
\hline $\mathrm{C}$ & 3.53023900 & 0.45697700 & -0.01852700 \\
\hline $\mathrm{H}$ & 2.51252100 & 1.06093900 & -1.84849500 \\
\hline $\mathrm{H}$ & 4.15728000 & -0.39171400 & 1.88491800 \\
\hline $\mathrm{H}$ & -0.25837000 & -1.94804200 & 1.86519800 \\
\hline $\mathrm{N}$ & -4.11489600 & 1.78382100 & 0.34557200 \\
\hline C & -4.49497600 & 2.55050300 & -0.83710100 \\
\hline $\mathrm{C}$ & -3.62745400 & 2.64837800 & 1.40322400 \\
\hline $\mathrm{H}$ & -5.25018600 & 3.29204000 & -0.55961600 \\
\hline $\mathrm{H}$ & -3.62863700 & 3.07795100 & -1.27410200 \\
\hline $\mathrm{H}$ & -4.92352700 & 1.90487600 & -1.60330600 \\
\hline $\mathrm{H}$ & -2.67513500 & 3.14756900 & 1.14699500 \\
\hline $\mathrm{H}$ & -4.37439400 & 3.42384900 & 1.59491600 \\
\hline $\mathrm{H}$ & -3.48787700 & 2.07640300 & 2.32419800 \\
\hline N & 4.71491200 & 1.11681100 & -0.28748600 \\
\hline $\mathrm{C}$ & 5.87593900 & 0.84080300 & 0.53656700 \\
\hline $\mathrm{C}$ & 4.93780900 & 1.65203000 & -1.61752000 \\
\hline $\mathrm{H}$ & 6.72012900 & 1.43018800 & 0.17652700 \\
\hline $\mathrm{H}$ & 5.69401700 & 1.12964000 & 1.57753900 \\
\hline $\mathrm{H}$ & 6.15965300 & -0.22310900 & 0.51737700 \\
\hline $\mathrm{H}$ & 4.20470000 & 2.43056700 & -1.85474900 \\
\hline $\mathrm{H}$ & 5.92645000 & 2.11093800 & -1.65809200 \\
\hline $\mathrm{H}$ & 4.88173800 & 0.87524500 & -2.39579400 \\
\hline
\end{tabular}

\begin{tabular}{|c|c|c|c|}
\hline $\mathrm{C}$ & 2.77111100 & 1.15211900 & -0.13511200 \\
\hline $\mathrm{C}$ & 1.54870300 & 0.52575900 & -0.15344900 \\
\hline C & 1.40198400 & -0.91034500 & -0.00629100 \\
\hline C & 2.63298300 & -1.75551400 & 0.10471800 \\
\hline C & 3.88566200 & -1.01992000 & 0.20011400 \\
\hline C & 3.95617800 & 0.32577500 & 0.09415500 \\
\hline $\mathrm{H}$ & 0.65345800 & 1.09591300 & -0.34666400 \\
\hline $\mathrm{H}$ & 4.78878000 & -1.60468900 & 0.33821900 \\
\hline $\mathrm{H}$ & 4.92965000 & 0.79660300 & 0.15640900 \\
\hline S & 2.67334500 & -3.41925400 & 0.13522300 \\
\hline C & 0.18177500 & -1.53628100 & 0.01093000 \\
\hline $\mathrm{C}$ & -1.15502400 & -0.94264800 & 0.03384300 \\
\hline C & -1.47163800 & 0.23055100 & 0.75269700 \\
\hline C & -2.19700000 & -1.61924700 & -0.63778800 \\
\hline C & -2.76695200 & 0.73197900 & 0.76768400 \\
\hline $\mathrm{H}$ & -0.70254800 & 0.72387900 & 1.33536700 \\
\hline C & -3.49238600 & -1.11924100 & -0.64684500 \\
\hline $\mathrm{H}$ & -1.97520500 & -2.53906300 & -1.17025700 \\
\hline C & -3.75797500 & 0.05830100 & 0.05307200 \\
\hline $\mathrm{H}$ & -3.02527400 & 1.62267100 & 1.32754100 \\
\hline $\mathrm{H}$ & -4.29366500 & -1.62021200 & -1.17626900 \\
\hline $\mathrm{H}$ & 0.20423400 & -2.62409900 & -0.01318800 \\
\hline $\mathrm{N}$ & 2.93470600 & 2.50168900 & -0.35806900 \\
\hline $\mathrm{C}$ & 4.16365600 & 3.17112200 & 0.04454700 \\
\hline $\mathrm{C}$ & 1.74547800 & 3.31714000 & -0.50558800 \\
\hline
\end{tabular}




$\begin{array}{lr}\mathrm{H} & 4.07635800 \\ \mathrm{H} & 4.36560000 \\ \mathrm{H} & 5.02470200 \\ \mathrm{H} & 1.13327600 \\ \mathrm{H} & 2.03495200 \\ \mathrm{H} & 1.12704500 \\ \mathrm{~N} & -5.12191300 \\ \mathrm{O} & -5.98401100 \\ \mathrm{O} & -5.32646200\end{array}$

$\begin{array}{rr}4.23341600 & -0.18572100 \\ 3.06826200 & 1.12032800 \\ 2.79154700 & -0.51325900 \\ 3.32696500 & 0.41025400 \\ 4.34091900 & -0.74432900 \\ 2.94311800 & -1.32845700 \\ 0.59239500 & 0.05536800 \\ -0.03461400 & -0.55063100 \\ 1.63923300 & 0.66128600\end{array}$

Sulphur-A6- $\left(\mathrm{NMe}_{2}, \mathrm{NO}_{2}\right)$, Closed Geometry

$\begin{array}{lrrr}\mathrm{C} & 3.24477600 & -0.59745400 & 0.10436400 \\ \mathrm{C} & 2.00357500 & -0.34480000 & 0.75273500 \\ \mathrm{C} & 1.38082200 & 0.86103000 & 0.49068700 \\ \mathrm{C} & 1.89290900 & 1.80334200 & -0.38906200 \\ \mathrm{C} & 3.09000300 & 1.59022400 & -1.05418000 \\ \mathrm{C} & 3.75046700 & 0.38140200 & -0.79372700 \\ \mathrm{H} & 1.55025700 & -1.06363300 & 1.42260300 \\ \mathrm{H} & 3.51030000 & 2.30276500 & -1.75528800 \\ \mathrm{H} & 4.68174600 & 0.20082600 & -1.31361900 \\ \mathrm{~S} & 0.53137800 & 2.98515800 & -0.25892200 \\ \mathrm{C} & 0.08499200 & 1.53521900 & 0.90617600 \\ \mathrm{C} & -1.19778600 & 0.83076000 & 0.55577300 \\ \mathrm{C} & -1.29714900 & 0.10147400 & -0.64046700 \\ \mathrm{C} & -2.30271100 & 0.90018200 & 1.41250300 \\ \mathrm{C} & -2.47469700 & -0.55367000 & -0.97424000 \\ \mathrm{H} & -0.43987500 & 0.05535000 & -1.30495300 \\ \mathrm{C} & -3.49376700 & 0.24862000 & 1.09371700 \\ \mathrm{H} & -2.23443400 & 1.46565600 & 2.33699100 \\ \mathrm{C} & -3.55819500 & -0.47004600 & -0.09635700 \\ \mathrm{H} & -2.57044200 & -1.12381100 & -1.88999700 \\ \mathrm{H} & -4.35868600 & 0.28702800 & 1.74460000 \\ \mathrm{H} & 0.08474400 & 1.89441500 & 1.93760900 \\ \mathrm{~N} & 3.94588700 & -1.76930000 & 0.34864700 \\ \mathrm{C} & 5.04513600 & -2.12314300 & -0.52953800 \\ \mathrm{C} & 3.26801900 & -2.84990900 & 1.03945700 \\ \mathrm{H} & 5.46421600 & -3.07847800 & -0.21127400 \\ \mathrm{H} & 4.73012200 & -2.21227300 & -1.58140000 \\ \mathrm{H} & 5.84551300 & -1.37791000 & -0.47121100 \\ \mathrm{H} & 2.36952200 & -3.19445500 & 0.50276400 \\ \mathrm{H} & 3.95141000 & -3.69309100 & 1.14752100 \\ \mathrm{H} & 2.96928000 & -2.53973400 & 2.04630800 \\ \mathrm{~N} & -4.80488700 & -1.16626900 & -0.43702900 \\ \mathrm{O} & -5.74854400 & -1.06504800 & 0.33902300 \\ \mathrm{O} & -4.83409100 & -1.81190000 & -1.47855800 \\ & & & \\ & & & \\ & & & \end{array}$

Sulphur-D4-(H, H), Open Geometry

$\begin{array}{lrrr}\text { C } & 1.89941400 & 2.32772700 & -0.34965600 \\ \mathrm{C} & 0.80270700 & 1.54050000 & -0.25436500 \\ \mathrm{C} & 0.91008500 & 0.09713500 & -0.06103200 \\ \mathrm{C} & 2.27221600 & -0.50869500 & 0.04225700 \\ \mathrm{C} & 3.39170100 & 0.40666600 & -0.09318700 \\ \mathrm{C} & 3.21814400 & 1.74454700 & -0.25576200 \\ \mathrm{H} & -0.18282500 & 1.96790100 & -0.39462900 \\ \mathrm{H} & 4.38629500 & -0.02240500 & -0.03771400 \\ \mathrm{H} & 4.08611000 & 2.39308300 & -0.33600000 \\ \mathrm{~S} & 2.56800100 & -2.12536500 & 0.35919300 \\ \mathrm{C} & -0.19391100 & -0.71464400 & -0.06019200 \\ \mathrm{C} & -1.60734700 & -0.33855800 & -0.00477600 \\ \mathrm{C} & -2.08635900 & 0.73866000 & 0.76824700 \\ \mathrm{C} & -2.53922500 & -1.13334700 & -0.70324600 \\ \mathrm{C} & -3.44745300 & 1.03375500 & 0.80357100 \\ \mathrm{H} & -1.39224500 & 1.31162700 & 1.37341400 \\ \mathrm{C} & -3.89656500 & -0.82280300 & -0.68299400 \\ \mathrm{H} & -2.18502800 & -1.98713200 & -1.27395200 \\ \mathrm{C} & -4.35430700 & 0.26450600 & 0.06788300 \\ \mathrm{H} & -3.80389900 & 1.85677400 & 1.41483800 \\ \mathrm{H} & -4.59902200 & -1.43324200 & -1.24133600\end{array}$




$\begin{array}{rrrr}\mathrm{H} & 0.00202800 & -1.78508500 & -0.11371500 \\ \mathrm{H} & -5.41368000 & 0.49924100 & 0.09505600 \\ \mathrm{H} & 1.79964400 & 3.39237400 & -0.53353800\end{array}$

$\begin{array}{lrrr}\text { Sulphur-D4-(H, H), Closed Geometry } & \\ \text { C } & 2.84938800 & -1.81960700 & 0.43589800 \\ \text { C } & 1.58197300 & -1.43455100 & 0.90818800 \\ \text { C } & 1.15436200 & -0.16093300 & 0.57224800 \\ \text { C } & 1.93015300 & 0.68759000 & -0.21324300 \\ \text { C } & 3.18619900 & 0.33621200 & -0.68803400 \\ \text { C } & 3.63026800 & -0.95076100 & -0.33907900 \\ \text { H } & 0.97203400 & -2.10887100 & 1.50113500 \\ \text { H } & 3.79074100 & 0.99960200 & -1.29616400 \\ \text { H } & 4.60407700 & -1.28346400 & -0.68558400 \\ \text { S } & 0.75599200 & 2.05197200 & -0.27754200 \\ \text { C } & -0.06965900 & 0.70582200 & 0.80809200 \\ \text { C } & -1.39639800 & 0.19213800 & 0.31740200 \\ \text { C } & -1.48775400 & -0.46924200 & -0.91647600 \\ \text { C } & -2.55103400 & 0.36538900 & 1.08717500 \\ \text { C } & -2.71363900 & -0.95150200 & -1.36697500 \\ \text { H } & -0.59031500 & -0.59504600 & -1.51575000 \\ \text { C } & -3.78303500 & -0.12058700 & 0.63667600 \\ \text { H } & -2.48836400 & 0.87830100 & 2.04311700 \\ \text { C } & -3.86622000 & -0.77937800 & -0.58989100 \\ \text { H } & -2.77496700 & -1.46163800 & -2.32317800 \\ \text { H } & -4.67214500 & 0.01596600 & 1.24422400 \\ \text { H } & -0.13555400 & 1.08493800 & 1.83067900 \\ \text { H } & -4.82086100 & -1.15761400 & -0.94167100 \\ \text { H } & 3.23186300 & -2.80729000 & 0.67197600 \\ & & & \end{array}$

\begin{tabular}{|c|c|c|c|}
\hline $\mathrm{C}$ & 2.89342400 & 1.23791100 & -0.09321400 \\
\hline C & 1.67798400 & 0.62176800 & -0.12315500 \\
\hline C & 1.58329200 & -0.80687400 & -0.03053100 \\
\hline C & 2.81950800 & -1.60688700 & 0.11508200 \\
\hline C & 4.06650200 & -0.86490100 & 0.08908800 \\
\hline C & 4.11410300 & 0.49196100 & 0.01282600 \\
\hline $\mathrm{H}$ & 0.79310700 & 1.21801000 & -0.30128200 \\
\hline $\mathrm{H}$ & 4.98288800 & -1.44015200 & 0.16219500 \\
\hline $\mathrm{H}$ & 5.05421400 & 1.03063600 & 0.01439100 \\
\hline S & 2.83791300 & -3.26488200 & 0.37174200 \\
\hline C & 0.35751100 & -1.45261500 & -0.19292400 \\
\hline C & -0.96963300 & -0.93478400 & -0.07797300 \\
\hline C & -1.32600300 & 0.21182900 & 0.68096600 \\
\hline C & -2.03011200 & -1.63631000 & -0.70984900 \\
\hline $\mathrm{C}$ & -2.62489100 & 0.66570300 & 0.74445300 \\
\hline $\mathrm{H}$ & -0.57155300 & 0.71718100 & 1.27325800 \\
\hline C & -3.33218300 & -1.18571100 & -0.67660300 \\
\hline $\mathrm{H}$ & -1.80204500 & -2.54494900 & -1.26057200 \\
\hline C & -3.67296500 & -0.00015900 & 0.03967900 \\
\hline $\mathrm{H}$ & -2.84363700 & 1.52774300 & 1.36030200 \\
\hline $\mathrm{H}$ & -4.09164600 & -1.75205000 & -1.19843900 \\
\hline $\mathrm{H}$ & 0.42811200 & -2.51062000 & -0.44601300 \\
\hline $\mathrm{N}$ & -4.94710200 & 0.46488200 & 0.07389200 \\
\hline $\mathrm{C}$ & -6.00873400 & -0.24547300 & -0.62127100 \\
\hline $\mathrm{C}$ & -5.26401000 & 1.68352800 & 0.80212700 \\
\hline $\mathrm{H}$ & -6.95016400 & 0.28243000 & -0.47321900 \\
\hline $\mathrm{H}$ & -5.81097800 & -0.29719700 & -1.69908100 \\
\hline $\mathrm{H}$ & -6.12379200 & -1.26607200 & -0.23630600 \\
\hline $\mathrm{H}$ & -4.67794700 & 2.53109000 & 0.42744300 \\
\hline $\mathrm{H}$ & -6.31999100 & 1.91750600 & 0.67084500 \\
\hline $\mathrm{H}$ & -5.06993800 & 1.57072700 & 1.87611000 \\
\hline $\mathrm{N}$ & 2.95851800 & 2.68346500 & -0.24044600 \\
\hline O & 1.90522100 & 3.31536800 & -0.31000500 \\
\hline 0 & 4.07154200 & 3.20231700 & -0.27797400 \\
\hline
\end{tabular}




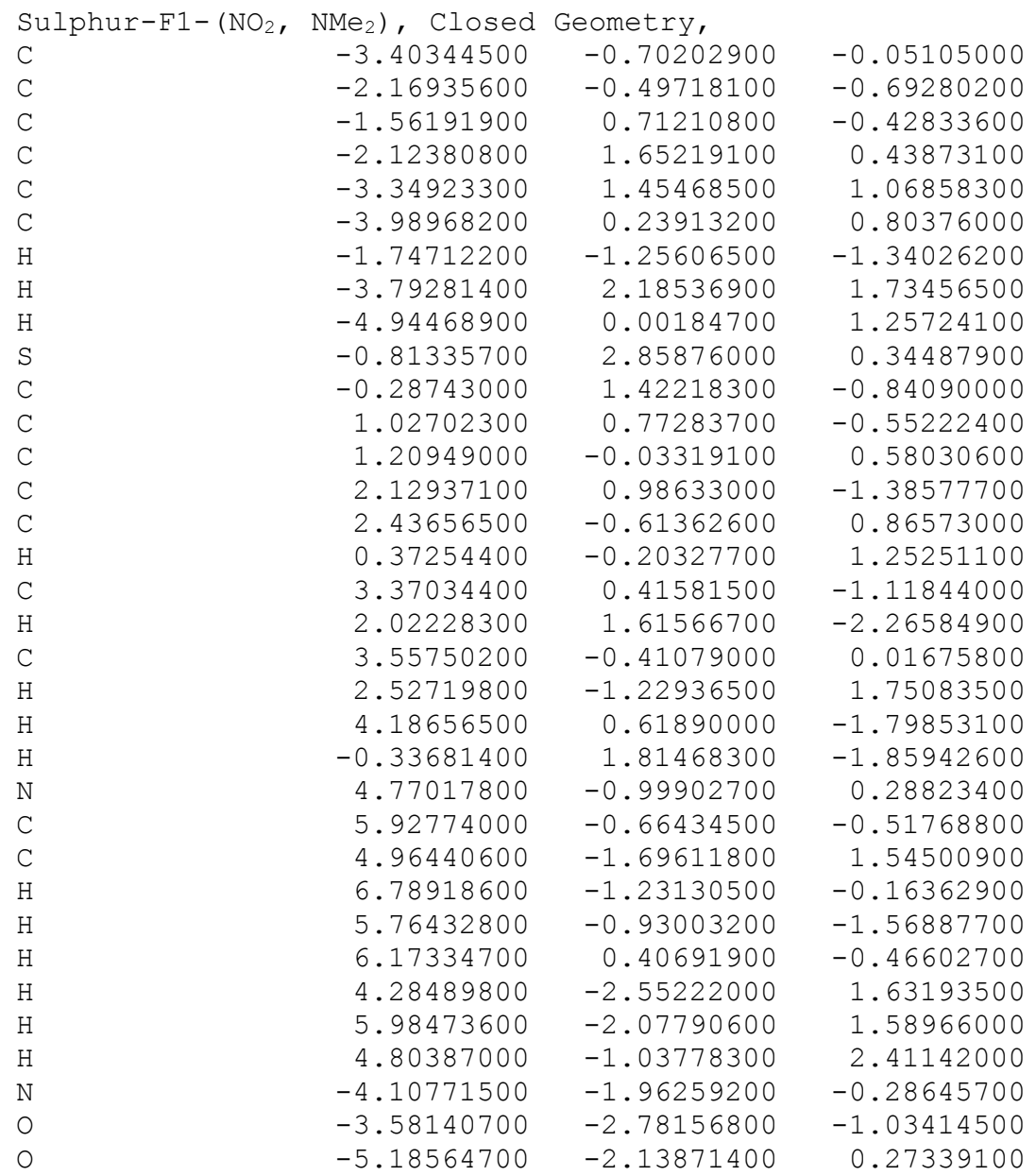

$\begin{array}{lrrr}\text { Sulphur-F6- }\left(\mathrm{NO}_{2},\right. & \left.\mathrm{NO}_{2}\right), \text { Open Geometry } & \\ \mathrm{C} & 2.72685600 & 1.26314000 & -0.08536200 \\ \mathrm{C} & 1.53634900 & 0.62513000 & -0.08879600 \\ \mathrm{C} & 1.48253100 & -0.82377800 & 0.00705000 \\ \mathrm{C} & 2.76930700 & -1.58671100 & 0.09711100 \\ \mathrm{C} & 3.99072100 & -0.79222100 & 0.07090000 \\ \mathrm{C} & 3.98467200 & 0.56031900 & 0.00109500 \\ \mathrm{H} & 0.63079300 & 1.20178000 & -0.22632700 \\ \mathrm{H} & 4.92886900 & -1.33321800 & 0.12461700 \\ \mathrm{H} & 4.90119400 & 1.13832300 & -0.00795700 \\ \mathrm{~S} & 2.86666100 & -3.23746700 & 0.27104200 \\ \mathrm{C} & 0.28879700 & -1.49222800 & -0.06008600 \\ \mathrm{C} & -1.06715400 & -0.94322400 & -0.01126200 \\ \mathrm{C} & -1.42665100 & 0.13434100 & 0.82437400 \\ \mathrm{C} & -2.06497600 & -1.56758900 & -0.78858000 \\ \mathrm{C} & -2.73444800 & 0.60541800 & 0.85058400 \\ \mathrm{H} & -0.68999100 & 0.57548700 & 1.48607600 \\ \mathrm{C} & -3.37082300 & -1.09320900 & -0.78619000 \\ \mathrm{H} & -1.80486100 & -2.41898600 & -1.40988000 \\ \mathrm{C} & -3.68133500 & -0.00850400 & 0.03309100 \\ \mathrm{H} & -3.03060400 & 1.42564700 & 1.49304400 \\ \mathrm{H} & -4.14557300 & -1.54684600 & -1.39217300 \\ \mathrm{H} & 0.35376300 & -2.57473600 & -0.16872600 \\ \mathrm{~N} & -5.06683700 & 0.48981800 & 0.05411500 \\ \mathrm{O} & -5.88180500 & -0.07065400 & -0.66736000 \\ \mathrm{O} & -5.31933900 & 1.43461900 & 0.79002100 \\ \mathrm{~N} & 2.74942500 & 2.72304700 & -0.22465600 \\ \mathrm{O} & 1.67889500 & 3.31790100 & -0.27459300 \\ \mathrm{O} & 3.84833000 & 3.26271700 & -0.27620800\end{array}$




$\begin{array}{lrrr}\text { Sulphur-F6- }\left(\mathrm{NO}_{2}, \mathrm{NO}_{2}\right), \text { Closed Geometry } \\ \mathrm{C} & 3.27169100 & -0.70488700 & \\ \mathrm{C} & 2.04961600 & -0.51263400 & 0.62942300 \\ \mathrm{C} & 1.44705700 & 0.70735700 & 0.46977700 \\ \mathrm{C} & 2.00510200 & 1.66549900 & -0.37824400 \\ \mathrm{C} & 3.21654000 & 1.48087700 & -1.03435500 \\ \mathrm{C} & 3.85129200 & 0.25349600 & -0.80995800 \\ \mathrm{H} & 1.63723900 & -1.28761100 & 1.33021300 \\ \mathrm{H} & 3.65350800 & 2.22596800 & -1.68840500 \\ \mathrm{H} & 4.79745000 & 0.02073800 & -1.28401600 \\ \mathrm{~S} & 0.69740800 & 2.87840000 & -0.22126400 \\ \mathrm{C} & 0.17935200 & 1.42080700 & 0.90416400 \\ \mathrm{C} & -1.13544100 & 0.77611300 & 0.55589600 \\ \mathrm{C} & -1.30693200 & 0.14836600 & -0.68804100 \\ \mathrm{C} & -2.19434100 & 0.80006000 & 1.47028000 \\ \mathrm{C} & -2.51525200 & -0.45281600 & -1.01516200 \\ \mathrm{H} & -0.48624300 & 0.13874000 & -1.39880800 \\ \mathrm{C} & -3.41457900 & 0.19976500 & 1.15915300 \\ \mathrm{H} & -2.06864300 & 1.28484800 & 2.43358300 \\ \mathrm{C} & -3.55196200 & -0.41666500 & -0.08017400 \\ \mathrm{H} & -2.66968900 & -0.94343500 & -1.96822700 \\ \mathrm{H} & -4.24611100 & 0.20181800 & 1.85319400 \\ \mathrm{H} & 0.20867600 & 1.75569500 & 1.94332700 \\ \mathrm{~N} & -4.83425900 & -1.05363100 & -0.41658400 \\ \mathrm{O} & -5.72756200 & -1.01405200 & 0.42060700 \\ \mathrm{O} & -4.93489900 & -1.58631300 & -1.51471100 \\ \mathrm{~N} & 3.97331900 & -1.97797900 & 0.22163700 \\ \mathrm{O} & 3.44850600 & -2.81236900 & 0.95125800 \\ \mathrm{O} & 5.04258200 & -2.14000400 & -0.35530900 \\ & & & \end{array}$




\subsubsection{Summary: Thermochemistry, $U_{A}$ and $U_{R}$}

The results of the simplified scan procedure to approximate the total reaction energy $U_{R}$ and activation energy $U_{A}$ are summarized in the following tables. They are the basis for Figure 2 in the main text.

Table $\mathbf{S 1 0} . \mathrm{U}_{\mathrm{A}}$ as calculated from scan procedures

\begin{tabular}{cc|ccccccc}
\hline \multicolumn{2}{c|}{$\mathrm{U}_{\mathrm{A}}[\mathrm{kcal} / \mathrm{mol}]$} & \multicolumn{7}{|c}{$\mathrm{R}_{\text {benzo }}$} \\
\hline$\sigma$ & $\mathbf{R}_{\text {phenyl }}$ & $\mathrm{NMe}_{2}$ & $\mathrm{OEt}$ & $\mathrm{OMe}$ & $\mathrm{H}$ & $\mathrm{Br}$ & $\mathrm{CF}_{3}$ & $\mathrm{NO}_{2}$ \\
\hline-0.83 & $\mathrm{NMe}_{2}$ & 20.3 & 20.7 & 20.7 & 20.3 & 19.3 & 18.8 & 17.0 \\
-0.45 & $\mathrm{OEt}$ & 23.0 & 23.5 & 23.6 & 23.2 & 22.4 & 21.8 & 20.6 \\
-0.27 & $\mathrm{OMe}$ & 23.4 & 24.0 & 24.0 & 23.3 & 22.9 & 22.3 & 21.6 \\
0.00 & $\mathrm{H}$ & 25.6 & 26.6 & 26.8 & 26.1 & 25.8 & 25.5 & 24.7 \\
0.23 & $\mathrm{Br}$ & 25.9 & 27.0 & 27.1 & 26.4 & 26.2 & 25.7 & 24.9 \\
0.54 & $\mathrm{CF}_{3}$ & 27.6 & 28.7 & 28.7 & 28.0 & 27.9 & 27.8 & 26.9 \\
0.78 & $\mathrm{NO}_{2}$ & 27.3 & 29.2 & 29.5 & 28.9 & 28.8 & 28.6 & 28.0 \\
\hline
\end{tabular}

Table S11. $U_{R}$ as calculated from scan procedures

\begin{tabular}{cc|ccccccc}
\hline \multicolumn{2}{c|}{$\mathrm{U}_{\mathrm{R}}[\mathrm{kcal} / \mathrm{mol}]$} & \multicolumn{7}{|c}{$\mathrm{R}_{\text {benzo }}$} \\
\hline$\sigma$ & $\mathbf{R}_{\text {phenyl }}$ & $\mathrm{NMe}_{2}$ & $\mathrm{OEt}$ & $\mathrm{OMe}$ & $\mathrm{H}$ & $\mathrm{Br}$ & $\mathrm{CF}_{3}$ & $\mathrm{NO}_{2}$ \\
\hline-0.83 & $\mathrm{NMe}_{2}$ & 11.0 & 13.6 & 14.2 & 13.4 & 12.9 & 12.7 & 12.4 \\
-0.45 & $\mathrm{OEt}$ & 12.3 & 15.2 & 15.9 & 15.2 & 14.8 & 14.9 & 15.1 \\
-0.27 & $\mathrm{OMe}$ & 12.5 & 15.4 & 16.1 & 15.3 & 15.1 & 15.2 & 15.6 \\
0.00 & $\mathrm{H}$ & 13.3 & 16.4 & 17.2 & 16.6 & 16.4 & 16.7 & 17.5 \\
0.23 & $\mathrm{Br}$ & 13.5 & 16.7 & 17.5 & 17.0 & 16.7 & 17.0 & 17.8 \\
0.54 & $\mathrm{CF}_{3}$ & 14.2 & 17.2 & 18.0 & 17.5 & 17.3 & 18.1 & 18.8 \\
0.78 & $\mathrm{NO}_{2}$ & 13.9 & 17.4 & 18.2 & 17.9 & 17.7 & 18.3 & 19.3 \\
\hline
\end{tabular}




\subsubsection{Hammett-Brown correlation}

As discussed in the main text and as can also be seen from Figure 2, the linearizability of the activation energy using simple Hammett constants is poor due to the importance of resonance effects. However, the effect of the substituent $R_{p}$ on the activation energy can be linearized fairly well $\left(R^{2}>0.98\right.$ in all cases) for a fixed substituent $R_{b}$ using Brown's $\sigma^{+}$-values. ${ }^{11}$ This can be expected since Rp's resonance properties (+M/-M) directly impact the electron density in the forming carbenium p-orbital.

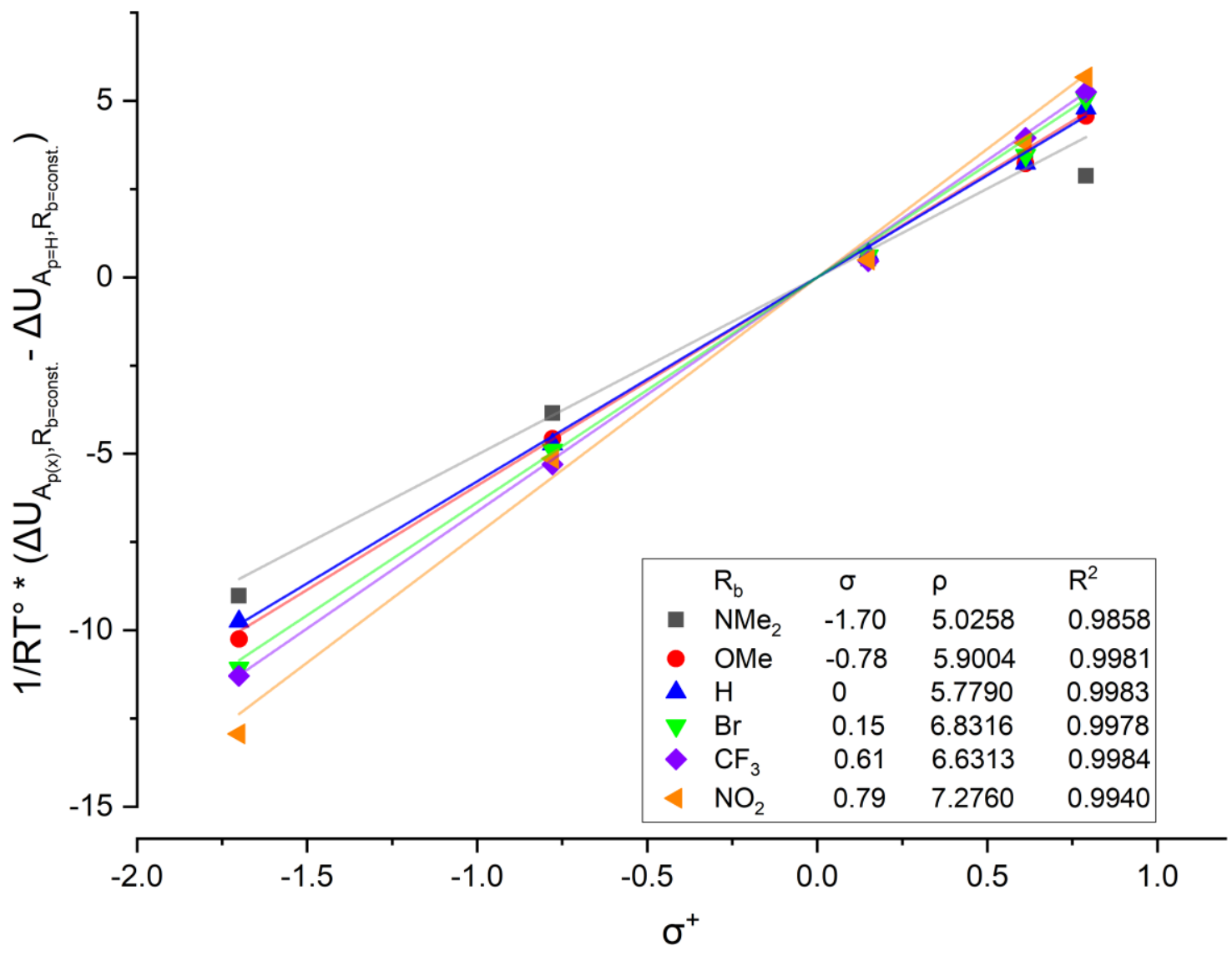

Figure S2. Hammett-Brown $\left(\sigma^{+}\right)$plot of $R_{p}$ against fixed substituents $R_{b} . R_{p}=H$ used as the respective reference.

The results underline the notion that electron-donating groups for $R_{p}$ increase the activation barrier. $\rho$ rises with decreasing electron-density on the benzo-ring, this is in accordance with the rationalization in the main text. Due to the dual role of $\mathrm{R}_{b}$ (para to the sulfur and meta to the benzylic carbon), this effect is more complicated and can only be poorly depicted by a single correlational approximation.

Note: We were unable to find a single set of data that contained all substituents used in our calculations. For reasons of consistency we decided to use constants that were determined by the same method, thus $\mathrm{R}_{\mathrm{p}}=\mathrm{OEt}$ had to be omitted. 


\subsubsection{Multi-correlational analysis}

\begin{tabular}{c|ccccccc}
\hline \multicolumn{7}{c}{ Logarithmic Rate Ratio: Computation } \\
\hline $\begin{array}{c}\text { 1/RT }\left(\mathrm{U}_{\mathrm{A}}-\mathrm{U}_{\mathrm{A}, \mathrm{H}}\right) \\
{[\mathrm{kcal} / \mathrm{mol}]}\end{array}$ & \multicolumn{7}{c}{$\mathrm{R}_{\text {benzo }}$} \\
\hline $\mathbf{R}_{\text {phenyl }}$ & $\mathrm{NMe}_{2}$ & $\mathrm{OEt}$ & $\mathrm{OMe}$ & $\mathrm{H}$ & $\mathrm{Br}$ & $\mathrm{CF}_{3}$ & $\mathrm{NO}_{2}$ \\
\hline $\mathrm{NMe}_{2}$ & -9.0 & -10.2 & -9.7 & -11.1 & -11.3 & -12.9 & -9.0 \\
$\mathrm{OMe}$ & -3.8 & -4.6 & -4.7 & -4.9 & -5.3 & -5.1 & -3.8 \\
$\mathrm{H}$ & 0.0 & 0.0 & 0.0 & 0.0 & 0.0 & 0.0 & 0.0 \\
$\mathrm{Br}$ & 0.5 & 0.6 & 0.7 & 0.6 & 0.5 & 0.5 & 0.5 \\
$\mathrm{CF}_{3}$ & 3.3 & 3.2 & 3.2 & 3.5 & 4.0 & 3.8 & 3.3 \\
$\mathrm{NO}_{2}$ & 2.9 & 4.6 & 4.8 & 5.1 & 5.2 & 5.7 & 2.9 \\
\hline
\end{tabular}

A multi-correlational analysis of the activation energy surface was attempted in the form of Young and Jencks' adaption of the Yukawa-Tsuno equation. ${ }^{12}$ We accounted for the different effects the $R_{b}$ group has on the stabilization of the sulfur and benzylic carbon respectively by using $\sigma^{+} / \sigma^{-}$. The results were unsatisfying, whether every substituent or even each group was attributed an individual slope $\rho$ and resonance factor $r$. The results are mathematically acceptable, but do not further the chemical understanding of the reaction. No sigma-scale (to be precise: model reaction) was found that accurately describes the orbital behavior we observed, consequentally linearization was not successful. $\rho_{p}$ values are dependant on the properties of $R_{\text {benzo, }}$ as can be seen in Figure $S 2$. This dependence cannot be corrected by using a set of linear combinations.

$\ln \frac{k_{p_{i}, b_{j}}}{k_{p_{i}, b_{H}}}=\frac{\Delta \Delta G^{\ddagger}}{R T}=\rho_{p_{i}}\left(\sigma_{p_{i}}+r_{p_{i}}\left(\sigma_{p_{i}}^{+}-\sigma_{p_{i}}\right)\right)+\rho_{m b_{i}}\left(\sigma_{m b_{i}}+r_{m b_{i}}\left(\sigma_{m b_{i}}^{+}-\sigma_{m b_{i}}\right)\right)+\rho_{p b_{i}}\left(\sigma_{p b_{i}}+r_{p b_{i}}\left(\sigma_{p b_{i}}^{-}-\sigma_{p b_{i}}\right)\right)$

Table S12. Young-Jencks-Yukawa-Tsuno multi-correlational approximation

\begin{tabular}{c|cccccc}
\hline \multicolumn{7}{c}{ Logarithmic Rate Ratio: Young-Jencks-Yukawa-Tsuno } \\
\hline $\begin{array}{c}\text { 1/RT }\left(\mathrm{U}_{\mathrm{A}}-\mathrm{U}_{\mathrm{A}, \mathrm{H}}\right) \\
{[\mathrm{kcal} / \mathrm{mol})}\end{array}$ & \multicolumn{7}{c}{$\mathrm{R}_{\text {benzo }}$} \\
\hline $\mathbf{R}_{\text {phenyl }}$ & $\mathrm{NMe}_{2}$ & $\mathrm{OMe}$ & $\mathrm{H}$ & $\mathrm{Br}$ & $\mathrm{CF}_{3}$ & $\mathrm{NO}_{2}$ \\
\hline $\mathrm{NMe}_{2}$ & -10.1 & -10.6 & -10.9 & -11.1 & -10.9 & -10.9 \\
$\mathrm{OMe}$ & -4.0 & -4.5 & -4.8 & -5.1 & -4.8 & -4.8 \\
$\mathrm{H}$ & 0.8 & 0.3 & 0.0 & -0.2 & 0.0 & 0.0 \\
$\mathrm{Br}$ & 1.3 & 0.8 & 0.5 & 0.3 & 0.5 & 0.5 \\
$\mathrm{CF}_{3}$ & 4.2 & 3.7 & 3.3 & 3.1 & 3.3 & 3.3 \\
$\mathrm{NO}_{2}$ & 5.6 & 5.1 & 4.8 & 4.6 & 4.8 & 4.8 \\
\hline$\Sigma \mathrm{RMS}^{7}$ & \multicolumn{7}{c}{17.0} \\
\hline
\end{tabular}


Table S13. Young-Jencks-Yukawa-Tsuno multi-correlational variables

\begin{tabular}{c|cccccc}
\hline $\mathrm{R}_{\mathrm{p}}$ & $\rho_{\mathrm{p}}$ & $\mathrm{r}_{\mathrm{p}}$ & $\rho_{\mathrm{pb}}$ & $\mathrm{r}_{\mathrm{pb}}$ & $\rho_{\mathrm{mb}}$ & $\mathrm{r}_{\mathrm{mb}}$ \\
\hline $\mathrm{NMe}_{2}$ & 6.32 & 1.03 & -1.00 & 1.00 & 0.00 & 0.15 \\
$\mathrm{OMe}$ & 6.33 & 0.97 & -1.00 & 1.00 & 0.00 & 0.49 \\
$\mathrm{H}$ & 6.00 & 1.00 & -1.00 & 1.00 & 1.00 & 1.00 \\
$\mathrm{Br}$ & 3.83 & 1.25 & -1.00 & 1.00 & 1.67 & 0.57 \\
$\mathrm{CF}_{3}$ & 5.48 & 0.97 & 0.00 & 1.00 & 0.82 & 0.00 \\
$\mathrm{NO}_{2}$ & 6.07 & 1.00 & 0.00 & 1.00 & 0.93 & 0.00 \\
\hline
\end{tabular}

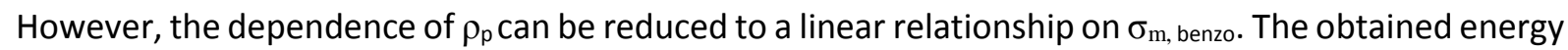
surface is in very good agreement with the computational results.

$$
\ln \frac{k_{p_{i}, b_{j}}}{k_{p_{i}, b_{H}}}=\frac{\Delta \Delta G^{\ddagger}}{R T}=\rho\left(\sigma_{m b}\right)\left(\sigma_{p_{i}}+r_{p_{i}}\left(\sigma_{p_{i}}^{+}-\sigma_{p_{i}}\right)\right)=\left(\alpha \sigma_{m b}+\beta\right)\left(\sigma_{p_{i}}+r_{p_{i}}\left(\sigma_{p_{i}}^{+}-\sigma_{p_{i}}\right)\right)
$$

Without further investigation, we want to refrain from physical or chemical interpretations of $\alpha$ and $\beta$ or why this relationship is a good approximation. However, this model allows us for a mathematical separation and thus comparison of the effects of $R_{\text {benzo }}$ and $R_{\text {phenyl. }}$ It should be noted that $\rho\left(R_{p}\right)>\alpha$ for all substituents $R_{p}$.

Table S14. $\rho_{\mathrm{p}}\left(\sigma_{\mathrm{m}}\right)$-linear relationship approximation

\begin{tabular}{c|cccccc}
\hline \multicolumn{7}{c}{ Logarithmic Rate Ratio: $\rho_{\mathrm{p}}\left(\sigma_{\mathrm{m}}\right.$, benzo)-linear relationship } \\
\hline $\begin{array}{c}\text { 1/RT } \\
\left(\mathrm{U}_{\mathrm{A}}-\mathrm{U}_{\mathrm{A}, \mathrm{H}}\right) \\
{[\mathrm{kcal} / \mathrm{mol}]}\end{array}$ & \multicolumn{7}{c}{$\mathrm{R}_{\text {benzo }}$} \\
\hline $\mathbf{R}_{\text {phenyl }}$ & $\mathrm{NMe}_{2}$ & $\mathrm{OMe}$ & $\mathrm{H}$ & $\mathrm{Br}$ & $\mathrm{CF}_{3}$ & $\mathrm{NO}_{2}$ \\
\hline $\mathrm{NMe}_{2}$ & -9.2 & -9.6 & -9.7 & -10.6 & -11.1 & -13.1 \\
$\mathrm{OMe}$ & -4.4 & -4.5 & -4.6 & -4.9 & -5.1 & -6.1 \\
$\mathrm{H}$ & 0.0 & 0.0 & 0.0 & 0.0 & 0.0 & 0.0 \\
$\mathrm{Br}$ & 0.5 & 0.8 & 0.7 & 0.9 & 0.9 & 1.0 \\
$\mathrm{CF}_{3}$ & 2.9 & 3.4 & 3.2 & 3.8 & 3.9 & 4.5 \\
$\mathrm{NO}_{2}$ & 3.6 & 4.3 & 4.1 & 4.9 & 5.1 & 5.7 \\
\hline$\Sigma \mathrm{RMS}$ & \multicolumn{7}{c}{8.8} \\
\hline
\end{tabular}

Table S15. $\rho_{\mathrm{p}}\left(\sigma_{\mathrm{m}}\right)$-linear relationship approximation

\begin{tabular}{ccc|cc}
\hline $\mathrm{R}_{\mathrm{b}}$ & $\rho_{\mathrm{p}}$ & $\mathrm{r}$ & $\alpha$ & $\beta$ \\
\hline $\mathrm{NMe}_{2}$ & 4.51 & 1.40 & 2.96 & 5.13 \\
$\mathrm{OMe}$ & 5.43 & 1.08 & & \\
$\mathrm{H}$ & 5.13 & 1.23 & & \\
$\mathrm{Br}$ & 6.14 & 1.04 & & \\
$\mathrm{CF}_{3}$ & 6.41 & 1.04 & & \\
$\mathrm{NO}_{2}$ & 7.24 & 1.12 & & \\
\hline
\end{tabular}


4.2.13 Thermochemistry Comparison of $\mathrm{A} 1-\left(\mathrm{NMe}_{2}, \mathrm{NMe}_{2}\right), \mathrm{A2}-\left(\mathrm{NMe}_{2}, \mathrm{OiPr}\right), \mathrm{D} 4-(\mathrm{H}, \mathrm{H}), \mathrm{F} 1-$ $\left(\mathrm{NO}_{2}, \mathrm{NMe}_{2}\right), \mathrm{F} 6-\left(\mathrm{NO}_{2}, \mathrm{NO}_{2}\right)$

Table S16. Thermochemistry Comparison:Total Energy U

\begin{tabular}{cccccc}
\hline \multicolumn{6}{c}{ Total Energy U [Hartree] } \\
\hline Compound & closed & TS & open & $\Delta_{\mathrm{A}} \mathrm{U}[\mathrm{kcal} / \mathrm{mol}]$ & $\Delta_{\mathrm{R}} \mathrm{U}[\mathrm{kcal} / \mathrm{mol}]$ \\
\hline A1 & -1167.09 & -1167.06 & -1167.07 & 20.29 & 10.98 \\
A6 & -1237.38 & -1237.33 & -1237.35 & 26.66 & 14.81 \\
D4 & -899.26 & -899.21 & -899.23 & 26.05 & 16.56 \\
F1 & -1237.38 & -1237.35 & -1237.36 & 17.04 & 13.31 \\
F6 & -1308.19 & -1308.15 & -1308.16 & 28.01 & 19.32
\end{tabular}

Table S17. Thermochemistry Comparison: $\mathbf{G}_{\text {corr }}+\varepsilon_{0}$

\begin{tabular}{cccccc}
\hline \multicolumn{6}{c}{$\mathrm{G}_{\text {corr }}+\varepsilon_{0}[\mathrm{Hartree}]$} \\
\hline Compound & closed & TS & open & $\Delta_{\mathrm{A}} \mathrm{G}[\mathrm{kcal} / \mathrm{mol}]$ & $\Delta_{\mathrm{RG}}[\mathrm{kcal} / \mathrm{mol}]$ \\
\hline A1 & -1166.80 & -1166.77 & -1166.78 & 19.70 & 10.68 \\
A6 & -1237.42 & -1237.38 & -1237.40 & 26.68 & 14.19 \\
D4 & -899.11 & -899.06 & -899.08 & 25.53 & 15.99 \\
F1 & -1237.43 & -1237.40 & -1237.41 & 17.25 & 13.14 \\
F6 & -1308.05 & -1308.00 & -1308.02 & 27.70 & 18.61
\end{tabular}

Table S18. Thermochemistry Comparison: $\mathbf{H}_{\text {corr }}+\varepsilon_{0}$

\begin{tabular}{cccccc}
\hline \multicolumn{6}{c}{$\mathrm{H}_{\text {corr }}+\varepsilon_{0}[\mathrm{Hartree}]$} \\
\hline Compound & closed & TS & open & $\Delta_{\mathrm{A}} \mathrm{H}[\mathrm{kcal} / \mathrm{mol}]$ & $\Delta_{\mathrm{R}} \mathrm{H}[\mathrm{kcal} / \mathrm{mol}]$ \\
\hline A1 & -1166.73 & -1166.70 & -1166.72 & 19.60 & 11.73 \\
A6 & -1237.36 & -1237.32 & -1237.33 & 26.71 & 15.17 \\
D4 & -899.06 & -899.02 & -899.03 & 25.25 & 16.79 \\
F1 & -1237.36 & -1237.34 & -1237.34 & 16.89 & 13.45 \\
F6 & -1307.98 & -1307.94 & -1307.95 & 27.45 & 19.59
\end{tabular}


Table S19. Thermochemistry Comparison: $\Delta_{A} S ; \Delta_{R} S$.

\begin{tabular}{ccc}
\hline Compound & $\Delta_{\mathrm{A}} \mathrm{S}[\mathrm{kcal} / \mathrm{mol}]$ & $\Delta_{\mathrm{R}} \mathrm{S}[\mathrm{kcal} / \mathrm{mol}]$ \\
\hline A1 & $3.41 \mathrm{E}-04$ & $-3.52 \mathrm{E}-03$ \\
A6 & $-8.42 \mathrm{E}-05$ & $-3.27 \mathrm{E}-03$ \\
D4 & $9.34 \mathrm{E}-04$ & $-2.70 \mathrm{E}-03$ \\
F1 & $1.22 \mathrm{E}-03$ & $-1.06 \mathrm{E}-03$ \\
F6 & $8.12 \mathrm{E}-04$ & $-3.28 \mathrm{E}-03$
\end{tabular}




\subsubsection{IRC-MO Plot (Walsh Diagram)}

To construct the Walsh diagram that illustrates the development of the orbital energies over the reaction coordinate, single point calculations at each step produced by the IRC calculations were performed on the $\mathrm{D} 4-(\mathrm{H}, \mathrm{H})$ substituted benzothiotete and the oxygen core variant. The orbital energies were taken from the single point log files.

\subsubsection{Single Point Calculations}

The single point calculations performed on the geometry's produced by the IRC calculations were produced with the following protocol.

\subsubsection{Exemplary Single Point File}

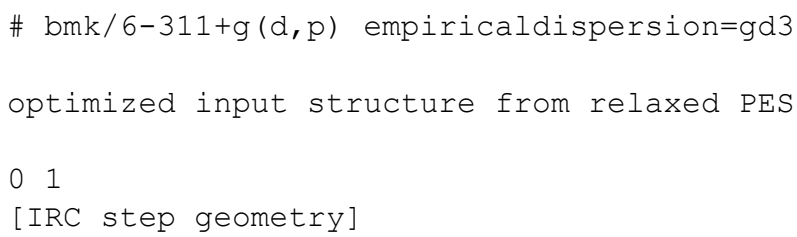

\subsubsection{Confirming Reliability of the Walsh Diagram}

The energies from each scan step and IRC step were compared to support the energetic trends in the molecular orbitals used in the Walsh Diagram. The difference in energy $(\Delta \mathrm{E})$ is relative to the lowest energy conformer produced by the scan.

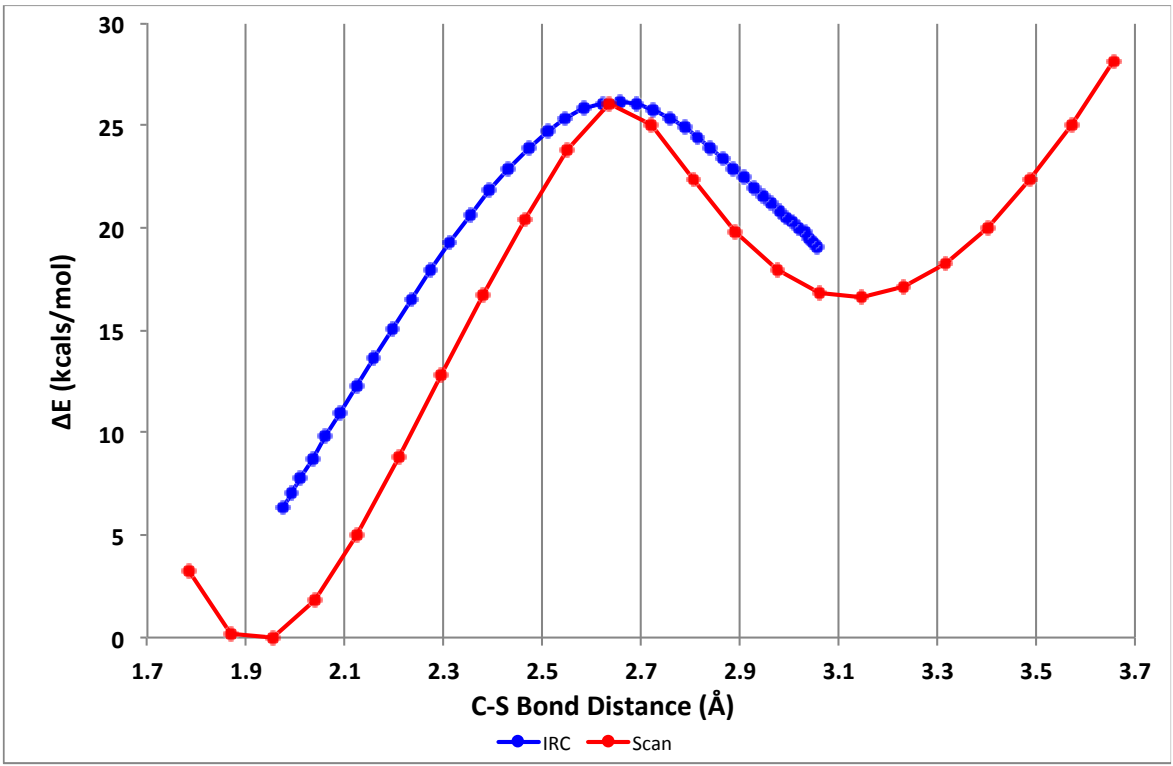

Figure S3. Comparison IRC vs scan. 


\subsubsection{Detailed Energy Diagram of Representative Benzothietes}

The transition state was calculated (as opposed to the usual scan-only procedure) and IRCs were conducted for 5 representative benzothietes. The results are very well within agreement with the shortened procedure $(<1.0 \mathrm{kcal} / \mathrm{mol}$ deviation).

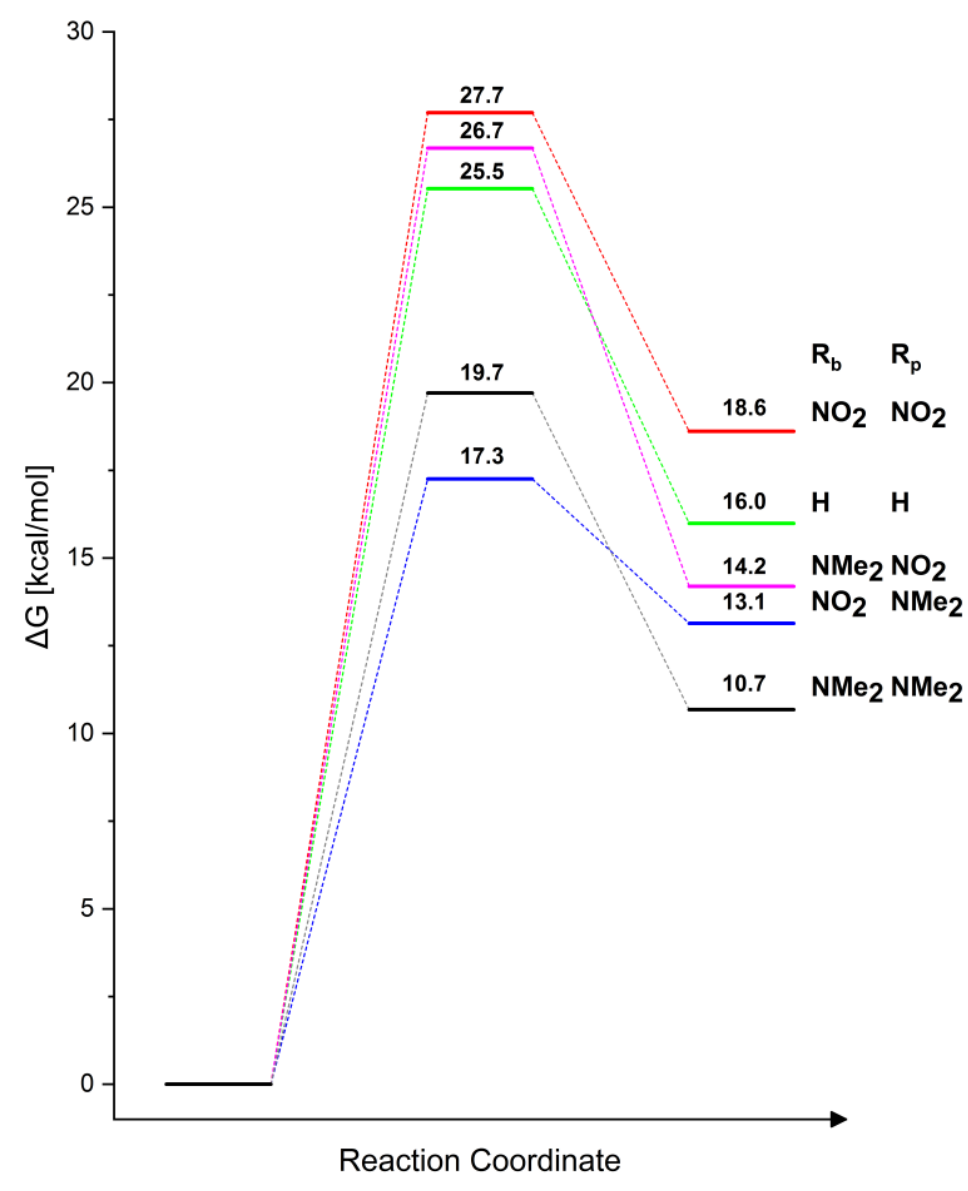

Figure S4. Detailed energy diagram of five representative benzothietes.

\subsubsection{Substituent Effects on Sulfur's Charge}

Optimizations with either the Benzo or Phenyl were substituted with the functional groups to explore the substituent effects on sulfur's charge. The charge on Sulfur was pulled from the last "Mulliken Charge" section of the log file. 


\subsubsection{Tables of Charges}

Closed Conformer

Table S20. Closed Conformer A

\begin{tabular}{ccccccc}
\hline$\#$ & $\mathbf{R}_{\text {benzo }}$ & $\mathbf{R}_{\text {phenyl }}$ & $\boldsymbol{\sigma}_{\text {phenyl }}$ & Sulfur Charge & HOMO $(\mathrm{eV})$ & LUMO $(\mathrm{eV})$ \\
\hline $\mathrm{F} 4$ & $\mathrm{NO}_{2}$ & $\mathrm{H}$ & 0.78 & -0.081674 & -0.27328 & -0.07504 \\
$\mathrm{E} 4$ & $\mathrm{CF}_{3}$ & $\mathrm{H}$ & 0.54 & -0.077816 & -0.26393 & -0.02370 \\
$\mathrm{G} 4$ & $\mathrm{Br}$ & $\mathrm{H}$ & 0.23 & -0.122489 & -0.25116 & -0.01993 \\
$\mathrm{D} 4$ & $\mathrm{H}$ & $\mathrm{H}$ & 0 & -0.103759 & -0.24745 & -0.01326 \\
$\mathrm{C} 4$ & $\mathrm{OMe}$ & $\mathrm{H}$ & -0.27 & -0.100884 & -0.23268 & -0.01106 \\
$\mathrm{~B} 4$ & $\mathrm{OiPr}$ & $\mathrm{H}$ & -0.45 & -0.088393 & -0.23032 & -0.01019 \\
$\mathrm{~A} 4$ & $\mathrm{NMe}_{2}$ & $\mathrm{H}$ & -0.83 & -0.11232 & -0.22117 & -0.00969
\end{tabular}

Table S21. Closed Conformer B

\begin{tabular}{ccccccc}
\hline$\#$ & $\mathbf{R}_{\text {benzo }}$ & $\mathbf{R}_{\text {phenyl }}$ & $\sigma_{\text {phenyl }}$ & Sulfur Charge & HOMO $(\mathrm{eV})$ & LUMO $(\mathrm{eV})$ \\
\hline $\mathrm{D} 6$ & $\mathrm{H}$ & $\mathrm{NO}_{2}$ & 0.78 & -0.126397 & -0.26307 & -0.08096 \\
$\mathrm{D} 5$ & $\mathrm{H}$ & $\mathrm{CF}_{3}$ & 0.54 & -0.095949 & -0.25726 & -0.03412 \\
$\mathrm{D} 7$ & $\mathrm{H}$ & $\mathrm{Br}$ & 0.23 & -0.114647 & -0.25243 & -0.02337 \\
$\mathrm{D} 4$ & $\mathrm{H}$ & $\mathrm{H}$ & 0 & -0.103759 & -0.24745 & -0.01326 \\
$\mathrm{D} 3$ & $\mathrm{H}$ & $\mathrm{OMe}$ & -0.27 & -0.187552 & -0.24220 & -0.00464 \\
$\mathrm{D} 2$ & $\mathrm{H}$ & $\mathrm{OiPr}$ & -0.45 & -0.126786 & -0.23994 & -0.00305 \\
$\mathrm{D} 1$ & $\mathrm{H}$ & $\mathrm{NMe}_{2}$ & -0.83 & -0.17459 & -0.22345 & 0.00081
\end{tabular}


Table S22. Open Conformer A

\begin{tabular}{ccccccc}
\hline$\#$ & $\mathbf{R}_{\text {benzo }}$ & $\mathbf{R}_{\text {phenyl }}$ & $\sigma_{\text {benzo }}$ & Sulfur Charge & HOMO (ev) & LUMO (ev) \\
\hline F4 & $\mathrm{NO}_{2}$ & $\mathrm{H}$ & 0.78 & -0.718198 & -0.26127 & -0.12549 \\
$\mathrm{E} 4$ & $\mathrm{CF}_{3}$ & $\mathrm{H}$ & 0.54 & -0.735889 & -0.25433 & -0.11529 \\
$\mathrm{G} 4$ & $\mathrm{Br}$ & $\mathrm{H}$ & 0.23 & -0.727988 & -0.24502 & -0.1104 \\
$\mathrm{D} 4$ & $\mathrm{H}$ & $\mathrm{H}$ & 0 & -0.742951 & -0.23936 & -0.10246 \\
$\mathrm{C} 4$ & $\mathrm{OMe}$ & $\mathrm{H}$ & -0.27 & -0.752069 & -0.22612 & -0.10129 \\
$\mathrm{~B} 4$ & $\mathrm{OiPr}$ & $\mathrm{H}$ & -0.45 & -0.752206 & -0.2279 & -0.09977 \\
$\mathrm{~A} 4$ & $\mathrm{NMe}_{2}$ & $\mathrm{H}$ & -0.83 & -0.741049 & -0.2235 & -0.09509
\end{tabular}

Table S23. Open Conformer B

\begin{tabular}{ccccccc}
\hline$\#$ & $\mathbf{R}_{\text {benzo }}$ & $\mathbf{R}_{\text {phenyl }}$ & $\sigma_{\text {benzo }}$ & Sulfur Charge & HOMO (ev) & LUMO (ev) \\
\hline D6 & $\mathrm{H}$ & $\mathrm{NO}_{2}$ & 0.78 & -0.734375 & -0.25752 & -0.12374 \\
D5 & $\mathrm{H}$ & $\mathrm{CF}_{3}$ & 0.54 & -0.738203 & -0.25074 & -0.11333 \\
D7 & $\mathrm{H}$ & $\mathrm{Br}$ & 0.23 & -0.727013 & -0.24342 & -0.10779 \\
D4 & $\mathrm{H}$ & $\mathrm{H}$ & 0 & -0.742951 & -0.23936 & -0.10246 \\
D3 & $\mathrm{H}$ & $\mathrm{OMe}$ & -0.27 & -0.790048 & -0.22898 & -0.09642 \\
D2 & $\mathrm{H}$ & $\mathrm{OiPr}$ & -0.45 & -0.767302 & -0.22595 & -0.09435 \\
D1 & $\mathrm{H}$ & $\mathrm{NMe}_{2}$ & -0.83 & -0.795635 & -0.21402 & -0.08781
\end{tabular}




\subsubsection{Oxygen_D4-(H, H) vs Sulfur_D4-(H, H) Reaction Coordinate}

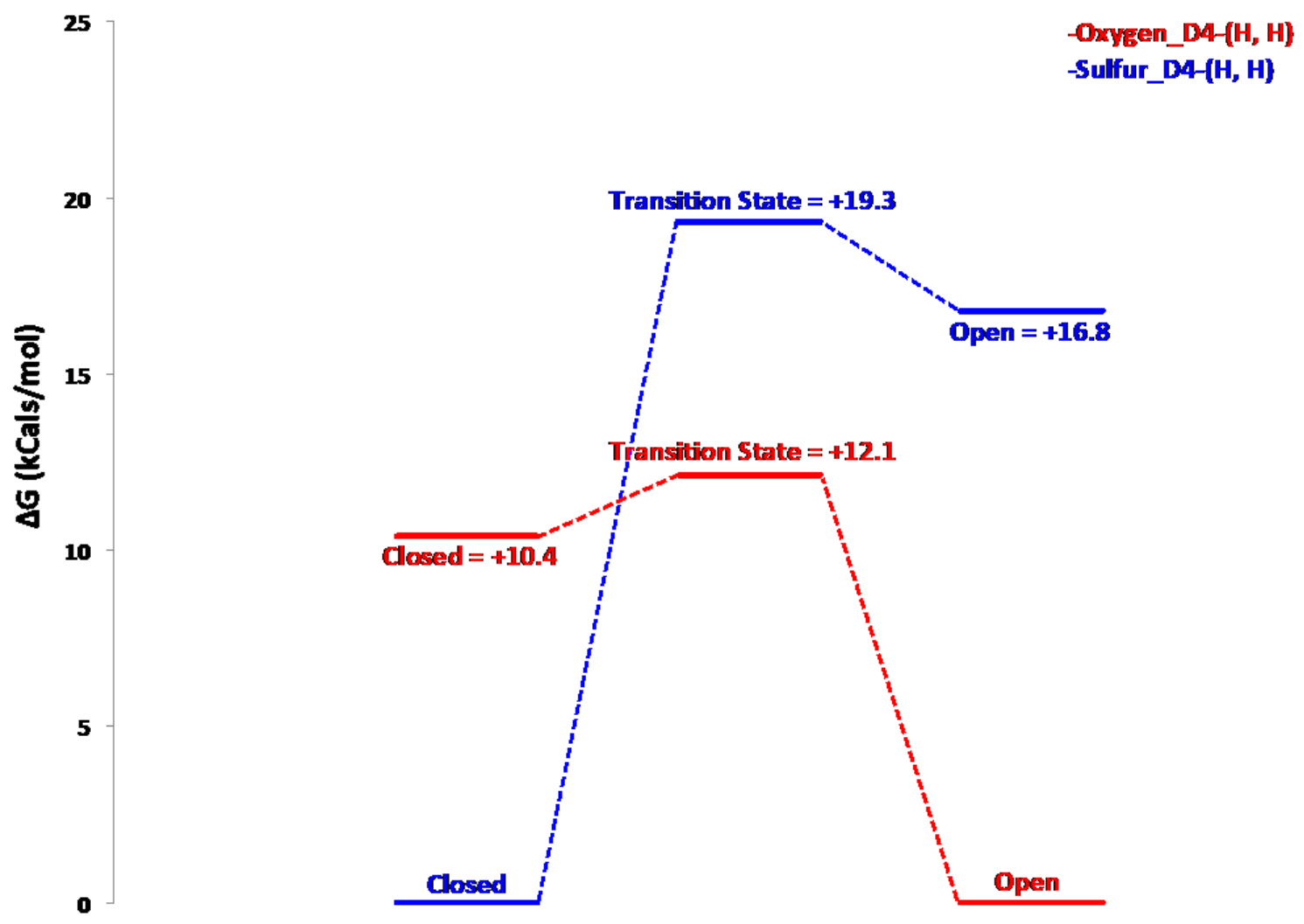

Figure S5. Comparison of D4-oxygen vs D4-sulfur reaction coordinate.

\subsubsection{Homodesmic Reaction Scheme}

For the calculation of the ring strain of the benzothiet/benzoxet a homodesmic reaction scheme was applied. As for all other thermochemical calculations, the BMK 6-311+g(d,p) (with dispersion) level of theory was applied.

Scheme S2. Homodesmic Reaction Scheme.<smiles>[R6]c1ccc(C2c3cc([R6])ccc3C2C)cc1</smiles><smiles>[R6]c1ccc(C(C)c2cc([R6])ccc2F)cc1</smiles> 


\subsubsection{Molecular Structures}

Oxygen_D4-(H, H)

Open Closed

Figure S6. D4-Oxygen structures.

Sulfur_D4-(H, H)

Open Closed

Figure S7. D4-Sulfur structures.

\subsubsection{Representative key MOs of Sulfur-D4}

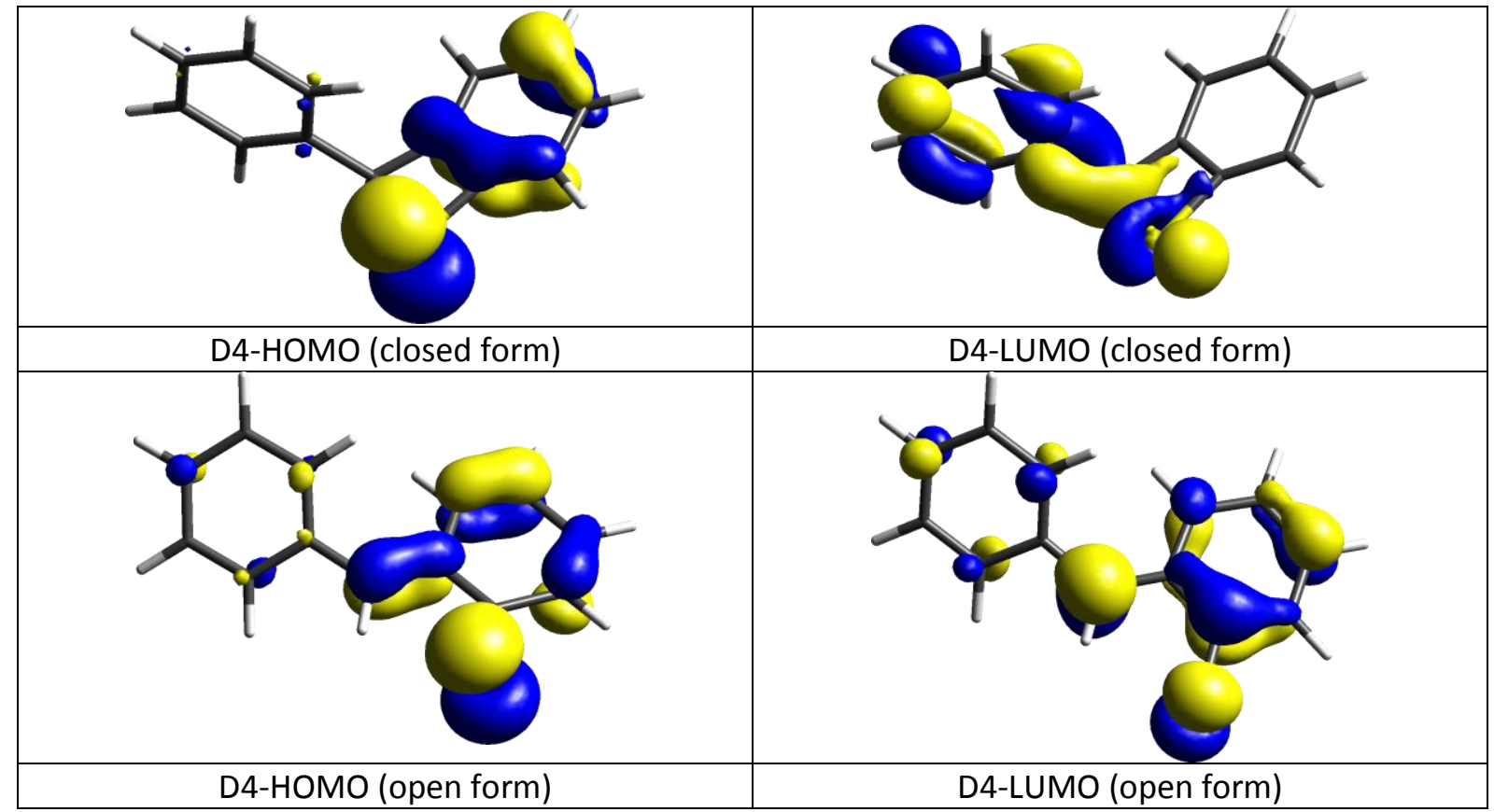

Figure S8. Key MOs of D4. 


\section{References}

(1) Krinsky, J. L.; Arnold, J.; Bergman, R. G. Organometallics 2007, 26, 897-909.

(2) Kobayashi, K.; Nakamura, D.; Miyamoto, K.; Morikawa, O.; Konishi, H. Bull. Chem. Soc. Jpn. 2007, $80,1780-1784$.

(3) Katritzky, A. R.; Kirichenko, K.; Ji, Y.; Prakash, I. Chem. Heterocycl. Compd. 2002, 38, 156-164.

(4) Streit, U.; Birbaum, F.; Quattropani, A.; Bochet, C. G. J. Org. Chem. 2013, 78, 6890-6910.

(5) Romagnoli, R.; Baraldi, P. G.; Carrion, M. D.; Lopez Cara, C.; Preti, D.; Fruttarolo, F.; Pavani, M. G.; Tabrizi, M. A.; Tolomeo, M.; Grimaudo, S.; Di Cristina, A.; Balzarini, J.; Hadfield, J. A.; Brancale, A.; Hamel, E. J. Med. Chem 2007, 50, 2273-2277.

(6) Hui, J. K.-H.; Yu, Z.; MacLachlan, M. J. Angew. Chem. 2007, 119, 8126-8129.

(7) Romagnoli, R.; Baraldi, P. G.; Carrion, M. D.; Cruz-Lopez, O.; Tolomeo, M.; Grimaudo, S.; Di Cristina, A.; Pipitone, M. R.; Balzarini, J.; Brancale, A.; Hamel, E. Bioorg Med Chem 2010, 18, 5114 5122.

(8) Ann, J.; Jung, A.; Kim, M.-Y.; Kim, H.-M.; Ryu, H.; Kim, S.; Kang, D. W.; Hong, S.; Cui, M.; Choi, S.; Blumberg, P. M.; Frank-Foltyn, R.; Bahrenberg, G.; Stockhausen, H.; Christoph, T.; Lee, J. Bioorg Med Chem 2015, 23, 6844-6854.

(9) Wu, R.-J.; Zhou, K.-X.; Yang, H.; Song, G.-Q.; Li, Y.-H.; Fu, J.-X.; Zhang, X.; Yu, S.-J.; Wang, L.-Z.; Xiong, L.-X.; Niu, C.-W.; Song, F.-H.; Yang, H.; Wang, J.-G. Eur J Med Chem 2019, 167, 472-484.

(10) Okuma, K.; Shiki, K.; Sonoda, S.; Koga, Y.; Shioji, K.; Kitamura, T.; Fujiwara, Y.; Yokomori, Y. Bull. Chem. Soc. Jpn. 2000, 73, 155-161.

(11) (a) Brown, H. C.; Okamoto, Y. J. Am. Chem. Soc. 1957, 79, 1913-1917; (b) Page, M. I. The chemistry of enzyme action; Elsevier, Amsterdam, New York, New York, NY, U.S.A, 2010; (c) Okamoto, Y.; Brown, H. C. J. Org. Chem. 1957, 22, 485-494;

(12) (a) Nakagawa, Y.; Tsuno, T.; Nakajima, K.; Iwai, M.; Kawai, H.; Okawa, K. Bull. Chem. Soc. Jpn. 1972, 45, 1162-1167; (b) Tsuno, Y.; Fujio, M. The Yukawa-Tsuno Relationship in Carbocationic Systems. In Advances in Physical Organic Chemistry; Bethell, D., Ed. 32; Elsevier textbooks: s.I., 1999, pp 267-385; (c) Tsuno, Y.; Kusuyama, Y.; Sawada, M.; Fujii, T.; Yukawa, Y. Bull. Chem. Soc. Jpn. 1975, 48, 3337-3346; (d) Yukawa, Y.; Tsuno, Y. Bull. Chem. Soc. Jpn. 1959, 32, 965-971; (e) Yukawa, Y.; Tsuno, Y.; Sawada, M. Bull. Chem. Soc. Jpn. 1966, 39, 2274-2286; (f) Young, P. R.; Jencks, W. P. J. Am. Chem. Soc. 1977, 99, 8238-8248; 